\title{
Rheology of Polymers Near Liquid-Solid Transitions
}

\author{
Horst Henning Winter and Marian Mours \\ University of Massachusetts, Department of Chemical Engineering and \\ Department of Polymer Science and Engineering, Amherst, MA 01003, USA
}

Polymeric materials near the liquid-solid transition (LST) exhibit a very distinct relaxation pattern. The reference point for analyzing these patterns is the instant of LST at which relaxation becomes self-similar over wide ranges of the relaxation time. The universality of this transition and its consequences have been explored extensively during the past decade. This study will present an overview of rheological implications inherent in liquid-solid transitions of polymers. The LST can be most reliably detected in a dynamic mechanical experiment in which the frequency independence of the loss tangent marks the LST. A wide variety of rheological observations of materials in the vicinity of an LST are discussed with respect to their universality. It is shown that polymer chemistry, molecular weight, stoichiometry, temperature, inhomogeneities, etc. greatly influence the material behavior near the LST. However, the characteristic self-similar relaxation is shown by all investigated materials, independent of the nature of the LST (e.g., both, physically and chemically crosslinking polymers). Several theories predict chemical and rheological properties in the vicinity of an LST. They are briefly discussed and compared with experimental results. A variety of applications for polymers near LST are presented that either already exist or can be envisioned. The self-similar relaxation behavior which results in a power law relaxation spectrum and modulus is not restricted to materials near LST. Different classes of polymers are described that also show power law relaxation behavior. What makes the self-similar relaxation specific for materials at LST is its occurrence at long times with the longest relaxation time diverging to infinity.

List of Abbreviations

List of Symbols

1 Patterns of Relaxation Near the Liquid-Solid Transition . . . . . . . 170

1.1 Rheological Observations of a Liquid-Solid Transition . . . . . 172

1.2 Relaxation Time Spectrum . . . . . . . . . . . . . . . 174

1.3 Divergence of Longest Relaxation Time . . . . . . . . . . . . . . 175

1.4 Interrelation Between Critical Exponents . . . . . . . . . 177

2 Theory of Gelation . . . . . . . . . . . . . . . . . . . . 179

2.1 Branching Theories . . . . . . . . . . . . . . . . . . 179

2.2 Percolation Theory . . . . . . . . . . . . . . . . . 181

2.3 Scaling Near the LST . . . . . . . . . . . . . . . . . . . . . 182

2.4 Critical Gel as Fractal Structure . . . . . . . . . . . . . . . . 184

2.5 The Notion of Topology . . . . . . . . . . . . . . . . . 186

2.6 Kinetic Theory (Smoluchowski Equation) . . . . . . . . . . . . 187

2.7 Computer Simulations . . . . . . . . . . . . . . . . . . 189 
3 Viscoelastic Properties at and Around the Liquid-Solid Transition . . 189

3.1 Linear Viscoelasticity of Liquids and Solids . . . . . . . . . . . 189

3.2 Viscoelastic Material Functions of Critical Gels . . . . . . . . . 190

3.3 Viscoelastic Material Functions Near LST . . . . . . . . . . 193

4 Constitutive Modeling with the Critical Gel Equation . . . . . . . . . 194

4.1 The Critical Gel Equation . . . . . . . . . . . . . . . . . . . . 194

4.2 Linear Viscoelastic Modeling of Critical Gels . . . . . . . . . 195

5 Physical Gelation . . . . . . . . . . . . . . . . . . . . . . . . . . . . . . . . 200

5.1 Physical Network Systems . . . . . . . . . . . . . . . . . 202

5.2 Dynamic Glass Transition . . . . . . . . . . . . . . . 205

5.3 Liquid Crystalline Polymers at their Nematic-Smectic Transition 206

5.4 Suspensions .................... 206

6 Rheometry Near the Gel Point . . . . . . . . . . . . . . . . . 207

6.1 Oscillatory Shear . . . . . . . . . . . . . . . . . . . 208

6.2 Sample Mutation ........................ 211

6.3 Time-Temperature Superposition . . . . . . . . . . . . . . . . 213

6.4 Time-Cure Superposition . . . . . . . . . . . . . . . . . . . . 214

6.5 Growth Rate of Moduli . . . . . . . . . . . . . . . . . . . . . . . . . . . 214

6.6 Inhomogeneities . . . . . . . . . . . . . 216

7 Detection of the Liquid-Solid Transition _ . . . . . . . . . . . . 218

7.1 Diverging Rheological Properties . . . . . . . . . . . . . . . . 219

7.2 Monotonously Changing Properties . . . . . . . . . . . . . . . 219

7.3 Uniqueness of $\tan \delta$ Method . . . . . . . . . . . . . . . . 220

7.4 Determination of $S$ and $n \ldots \ldots 221$

8 Other Observations of Power Law Relaxation . . . . . . . . . . . . . 222

8.1 Self-Similar Relaxation with Negative Exponent Value . . . . . 223

8.2 Self-Similar Relaxation with Positive Exponent Value . . . . . 224

8.3 Observations of Self-Similar Relaxation Spectra . . . . . . . . 224

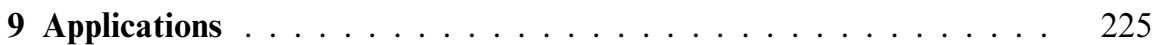

9.1 Avoiding the Gel Point . . . . . . . . . . . . . . . 225

9.2 Materials Near the Gel Point . . . . . . . . . . . . . . . . 226

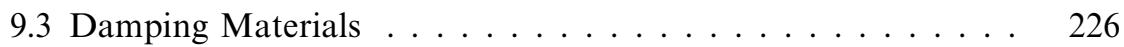

9.4 Pressure-Sensitive Adhesives . . . . . . . . . . . . . . . . 227

9.5 Processing Near the Physical Gel Point . . . . . . . . . . . . . . . . 228

9.6 Processing Near the Chemical Gel Point . . . . . . . . . . . 229

10 Conclusions . . . . . . . . . . . . . . . . . . . . . 229

11 References ........................... 230 


\section{List of Abbreviations}

BSW Baumgärtel, Schausberger, Winter (spectrum for linear flexible chains of uniform length) [61]

CW Chambon-Winter (spectrum for critical gel)

FS Flory-Stockmayer

LCP liquid crystalline polymer

LST liquid/solid transition

ODT order/disorder transition

PBD polybutadiene

PDMS polydimethylsiloxane

UV ultraviolet

\section{List of Symbols}

$\begin{array}{ll}A & \text { constant } \\ a_{T} & \text { horizontal shift factor } \\ \alpha_{-} & \text {critical exponent for longest relaxation time before LST } \\ \alpha_{+} & \text {critical exponent for longest relaxation time after LST } \\ b_{T} & \text { vertical shift factor } \\ \beta & \text { scaling exponent for gel fraction } \\ \mathbf{C}\left(t ; t^{\prime}\right) & \text { Cauchy strain tensor } \\ \mathbf{C}^{-1}\left(t ; t^{\prime}\right) & \text { Finger strain tensor } \\ c & \text { concentration } \\ d & \text { space dimension } \\ d_{\mathrm{f}} & \text { fractal dimension } \\ \bar{d}_{\mathrm{f}} & \text { fractal dimension with excluded volume screening } \\ \delta & \text { phase angle } \\ \delta_{\mathrm{c}} & \text { phase angle at LST } \\ E / R & \text { activation energy divided by universal gas constant } \\ F_{i j} & \text { fragmentation kernel, probability of cluster of size } \mathrm{i}+\mathrm{j} \text { to break } \\ & \text { up into cluster of size i and cluster of size } \mathrm{j} \\ f & \text { functionality, frequency } \\ \bar{f}_{2} & \text { average number of crosslinking sites along a chain } \\ f_{\mathrm{g}} & \text { gel fraction } \\ G & \text { relaxation modulus } \\ G_{0} & \text { plateau modulus of fully crosslinked material } \\ G_{\mathrm{e}} & \text { equilibrium modulus } \\ G^{*} & \text { complex modulus } \\ G^{\prime} & \text { storage modulus } \\ G^{\prime \prime} & \text { loss modulus }\end{array}$


$G_{\mathrm{c}}^{\prime}$

$G_{\mathrm{c}}^{\prime \prime}$

$\bar{g}_{2}$

$g$

$\Gamma$

$\gamma$

$M_{\mathrm{e}}$

$M_{\text {GAUSS }}$

$M_{\text {max }}$

$M_{\mathrm{n}}$

$M_{\mathrm{w}}$

m

$\mu$

$N$

$N(M)$

$N_{1}$

$N_{\mathrm{g}}$

$N_{\text {max }}$ storage modulus at LST

loss modulus at LST

average number of crosslinking sites along a chain

measured property in definition of mutation number

Gamma function

shear strain, critical exponent for molecular weight

step shear strain, shear strain amplitude

shear rate

constant shear rate

relaxation spectrum

front factor of power law spectrum

Heaviside step function

real part of complex viscosity, $\eta^{\prime}=G^{\prime \prime} / \omega$

imaginary part of complex viscosity, $\eta^{\prime \prime}=G^{\prime} / \omega$

zero-shear viscosity

creep compliance

equilibrium compliance

storage compliance

loss compliance

reaction kernel, probability of cluster of size $i$ to react with cluster

of size $\mathrm{j}$

scaling exponent of rates of change of dynamic moduli

exponent for certain reaction kernel $K_{i j}, \Lambda=\mu+v$

relaxation time

lower bound of $\mathrm{CW}$ relaxation spectrum, characteristic relaxation

time of liquid state, characteristic material time

lifetime of physical bond

characteristic relaxation time

lower cutoff relaxation time of power law spectrum

longest relaxation time

lifetime of physical cluster

upper cutoff relaxation time of power law spectrum

cluster mass

entanglement molecular weight

molecular weight above which chains behave Gaussian

molecular weight of largest cluster

number average molecular weight

weight average molecular weight

power law exponent (for spectrum with positive exponent)

exponent for homogeneous reaction kernel $K_{i j}$

number of bonds in a molecular cluster

cluster mass distribution

first normal stress difference

gel number

maximum number of bonds in a molecular cluster 


\begin{tabular}{|c|c|}
\hline$N_{\mathrm{mu}}$ & mutation number \\
\hline$n$ & relaxation exponent \\
\hline$n_{\mathrm{A} f}$ & number of molecules of $\mathrm{A}$ of functionality $\mathrm{f}$ \\
\hline$n_{\mathrm{B} g}$ & number of molecules of $\mathrm{B}$ of functionality $\mathrm{g}$ \\
\hline$n_{f}$ & number of molecules of functionality $\mathrm{f}$ \\
\hline$v$ & critical exponent for typical cluster size \\
\hline$p$ & $\begin{array}{l}\text { measure of connectivity, e.g. extent of reaction in case of chemical } \\
\text { gelation }\end{array}$ \\
\hline$p_{\mathrm{c}}$ & critical extent of reaction \\
\hline$p_{\mathrm{A}}$ & extent of reaction of species A \\
\hline$p_{\mathrm{B}}$ & extent of reaction of species B \\
\hline$\pi$ & osmotic pressure \\
\hline$R$ & radius of gyration \\
\hline$R_{\text {char }}$ & typical cluster size \\
\hline$r$ & molar ratio \\
\hline$r_{1}$ & lower molar ratio \\
\hline$r_{\mathrm{u}}$ & upper molar ratio \\
\hline$\rho$ & cluster density, mass density \\
\hline$S$ & gel stiffness \\
\hline$s$ & critical exponent for zero-shear viscosity \\
\hline$\sigma$ & critical exponent for maximum molecular weight \\
\hline$T$ & temperature \\
\hline$\Delta T$ & degree of supercooling \\
\hline$T_{0}$ & reference temperature \\
\hline$T_{\mathrm{c}}$ & temperature at critical point \\
\hline$T_{\mathrm{g}}$ & glass transition temperature \\
\hline$T_{\mathrm{m}}$ & melt temperature \\
\hline$t$ & time \\
\hline$\Delta t$ & sampling time \\
\hline$t^{\prime}$ & time (integration variable) \\
\hline$t^{\prime \prime}$ & time between $t^{\prime}$ and $t$ \\
\hline$t_{1}$ & creep time \\
\hline$t_{\mathrm{p}}$ & process time \\
\hline$\tau$ & critical exponent for cluster mass distribution, stress \\
\hline$\tau$ & stress tensor \\
\hline$\dot{\tau}$ & rate of change of shear stress \\
\hline$\tau_{0}$ & constant applied shear stress in creep experiment \\
\hline$\tau_{1}$ & stress under static load at infinite time \\
\hline$\tau_{11}-\tau_{22}$ & first normal stress difference \\
\hline$\tau_{21}=\tau_{12}$ & shear stress \\
\hline v & exponent for homogeneous reaction kernel $K_{i j}$ \\
\hline$\omega$ & angular frequency \\
\hline$\xi$ & correlation length \\
\hline$\psi_{1}$ & coefficient of first normal stress difference \\
\hline$z$ & critical exponent for equilibrium modulus \\
\hline
\end{tabular}




\section{Patterns of Relaxation Near the Liquid-Solid Transition}

Polymeric materials relax with a broad spectrum of relaxation modes. Magnitude and shape of the spectrum reflect the material structure in some complicated way. The longer modes belong to the motion of entire molecules or of large chain segments while the shorter modes characterize small scale details of the molecules. Extra long relaxation modes arise from large scale structures which some polymers are able to form due to phase separation or associations on the molecular or particulate level. The formation of such extensive clusters is the origin of many liquid-solid transitions. Most intriguing is the behavior near such transitions, when molecular motions slow down while they correlate with motions of other molecules over longer and longer distances. The relaxation modes are not independent any more, but they are somehow coupled over a wide range of time scales. This leads to a universal pattern of the relaxation time spectrum at liquid-solid transitions. The universality of the rheological behavior and its consequences have been explored extensively during the past decade, and this study will attempt to give an overview of the current state of the field.

There are many reasons for studying the liquid-solid transition (LST). The physicist might be interested in gelation as a critical phenomenon. The LST of polymers is also technically important since it occurs in nearly all of the common fabrication processes. Examples are injection molding of semi-crystalline polymers (where the surface quality of the finished parts may be affected by gelation) and processing of crosslinking polymers. The instant of LST has to be known for the design and operation of such polymer processing. The polymer processing engineer may like to anticipate the instant of gelation, often for the mere reason of avoiding or postponing it. Beyond that, processing near the gel point promises interesting texture development for ultimate material properties. The materials scientist might like to know the possible range of material properties close to the gel point. Conservation of the material state near an LST has the potential for novel properties which combine liquid and solid characteristics. Industrial applications are just beginning to explore such advantageous properties in adhesives, super absorbers, dampers, sealants, membranes, toner matrices, catalyst supports, etc. Gels are good adhesives since they combine the surface wetting property of liquids with the cohesive strength of solids. Strong adhesion and damping properties recommend gels as binder material in composite materials. Widespread technical applications have not yet materialized, since, until recently, it has been difficult to measure and control the LST. This has changed, and, as a consequence, one is able now to control processing near LST or to manufacture gels with reproducible properties.

The chemical gel point defines the instant of LST of chemically crosslinking polymers. Before the crosslinking polymer has reached its gel point it consists of a distribution of finite clusters. It is called a 'sol' since it is soluble in good solvents. Beyond the gel point, it is called a 'gel'. The gel is an infinitely large 
macromolecule which only can swell but not dissolve in a solvent, even if low molecular weight molecules (sol fraction) are still extractable from the gel. We will later borrow this terminology ('gel point', 'sol', 'gel') from chemical gelation and apply it to a wide range of materials which share rheological properties with chemically crosslinking systems. These are the physical gels, which are able to form extensive molecular or particulate clusters by a variety of different mechanisms. Examples are partially crystalline polymers, liquid crystalline polymers at their nematic-to-smectic transition, micro-phase separating block copolymers, and suspensions and emulsions at the percolation limit. Emphasis in this study will be on the rheological behavior, without trying to discuss the various 'crosslinking' mechanisms.

The independent variable, $p$, of the solidification process differs from material to material. It is a measure of connectivity (see Fig. 1), which requires restatement for each type of LST. An exception is chemical gelation for which the extent of crosslinking reaction, $p$, is defined and directly measurable as the ratio of the number of chemical bonds to the total number of possible bonds $(0<p<1$, without ever reaching unity), i.e. $p$ is the bond probability. At the critical extent of reaction, $p \rightarrow p_{\mathrm{c}}$, the molecular weight of the largest molecule diverges to infinity and the molecular weight distribution spreads infinitely broad $\left(M_{\mathrm{w}} / M_{\mathrm{n}} \rightarrow \infty\right)$, i.e. molecular sizes range from the smallest unreacted oligomer to the infinite cluster. This defines the gel point $[1,2]$. The value of $p_{\mathrm{c}}$ is not universal but depends on the details of the evolving structure.

The polymer at the gel point is in a critical state [3], and the name critical gel [4] is appropriate for distinguishing polymers at the gel point from the various materials which commonly are called gels. The critical gel has no intrinsic size scale except for the size of its oligomeric building block, and molecular motions are correlated over large distances. The combination of liquid and solid

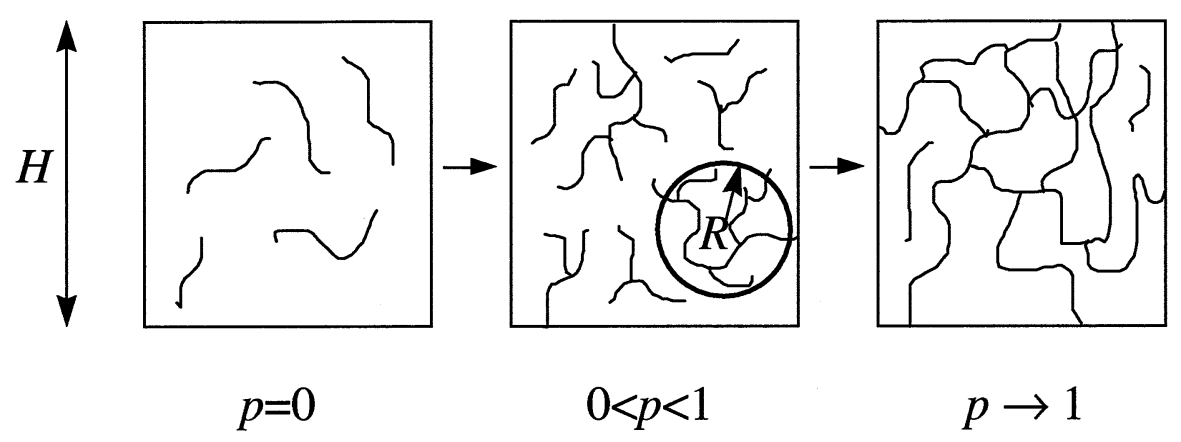

Fig. 1. Schematic of cluster growth during crosslinking. At $p=0$, only the monomer is present. With increasing crosslinking index, $p$, the connectivity increases and the molecular clusters (radius $R$ ) grow in size. In the solid state, the network spans the entire sample, $2 R \rightarrow H$ 
behavior at the gel point requires unusual simplicity and regularity in the relaxation pattern.

Very important are materials in the vicinity of the gel point. For such nearly critical gels, $p-p_{\mathrm{c}}$ is a measure of the distance from the gel point, and all properties can be expanded in powers of $\left|p-p_{\mathrm{c}}\right|$. This is permitted within the critical region at small distance at both sides of the gel point [3]. Material properties of nearly critical gels are still governed by the simplicity of the critical state. This changes at increased $\left|p-p_{\mathrm{c}}\right|$, where the behavior loses its simplicity. It will be interesting to study the rheological properties which gradually break free from that pattern as the distance from the gel point increases.

Material properties at a critical point were believed to be independent of the structural details of the materials. Such universality has yet to be confirmed for gelation. In fact, experiments show that the dynamic mechanical properties of a polymer are intimately related to its structural characteristics and forming conditions. A direct relation between structure and relaxation behavior of critical gels is still unknown since their structure has yet evaded detailed investigation. Most structural information relies on extrapolation onto the LST.

\subsection{Rheological Observations of a Liquid-Solid Transition}

The transition strongly affects the molecular mobility, which leads to large changes in rheology. For a direct observation of the relaxation pattern, one may, for instance, impose a small step shear strain $\gamma_{0}$ on samples near LST while measuring the shear stress response $\tau_{12}(t)$ as a function of time. The result is the shear stress relaxation function $G(t)=\tau_{12}(t) / \gamma_{0}$, also called relaxation modulus. Since the concept of a relaxation modulus applies to liquids as well as to solids, it is well suited for describing the LST.

Figure 2 shows a typical evolution of $G(t, p)$ near the LST of a crosslinking polymer. The $x$ axis shows the time of crosslinking reaction which corresponds to an extent of reaction, $p$. For each of the curves in Fig. 2, $p$ is kept constant. The crosslinking reaction was stopped at discrete values of $p$, which increased from sample to sample.

In samples with early stages of crosslinking (lower curves in Fig. 2), stress can relax quickly. As more and more chemical bonds are added, the relaxation process lasts longer and longer, i.e. $G(t)$ stretches out further and further. The downward curvature becomes less and less pronounced until a straight line ('power law') is reached at the critical point.

Exactly at the LST, the material behaves not as a liquid any more and not yet as a solid. The relaxation modes are not independent of each other but are coupled. The coupling is expressed by a power law distribution of relaxation modes [5-7]

$$
G(t)=S t^{-n} \text { for } \lambda_{0}<t<\infty
$$




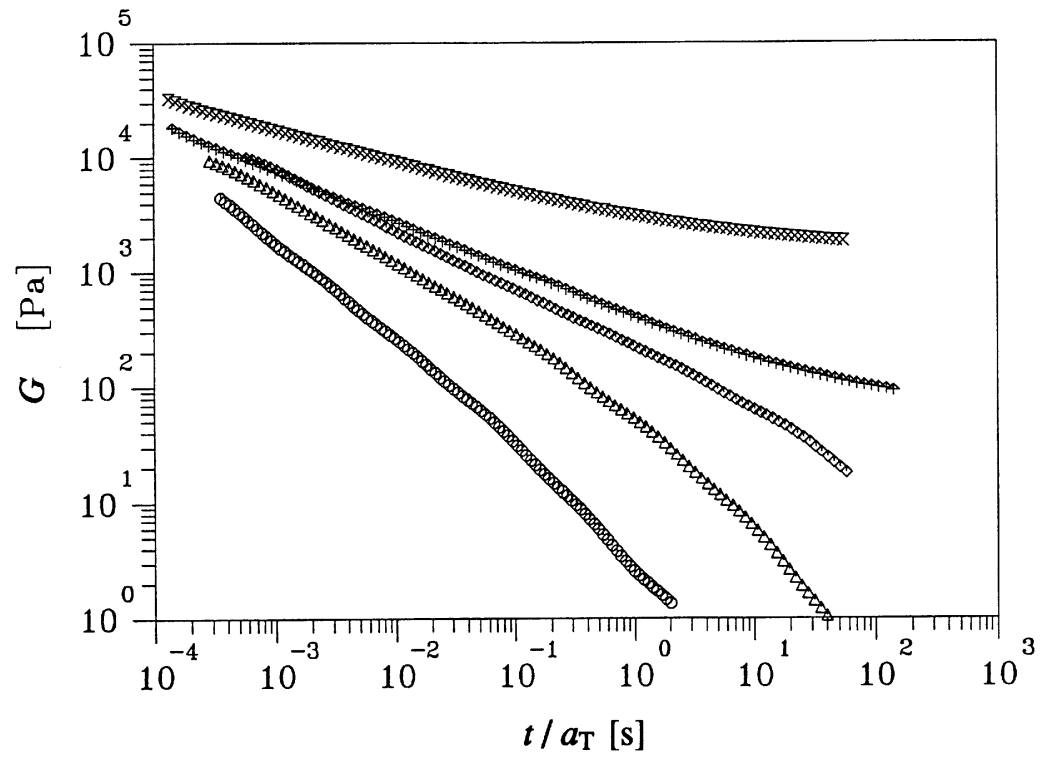

Fig. 2. Relaxation modulus $G(t)$ of a set of polydimethylsiloxane samples with increasing extent of crosslinking plotted against time of crosslinking. The linear PDMS chains $\left(M_{\mathrm{n}} \approx 10000\right.$, polydispersity index $\approx 2$ ) were endlinked with a four-functional silane crosslinker catalyzed by a platinum compound. Samples with different extent of reaction were prepared by poisoning the reaction at different times. The actual extent of reaction was not determined. Two of the samples are clearly before the gel point (LST) and two beyond. The third sample is very close to the gel point. Data of Chambon and Winter [5] evaluated by Baumgärtel and Winter [8]

$S$ being the gel stiffness as indicated by the straight line in the $\log / \log$ plot, Fig. 2 . This marks the intermediate state between curving down and curving to the right, and we assume that the power law behavior extends to infinite times. The power law may be explained by the hypothesis that one probes self-similar regions of the critical gel by varying the times of observations [4]. The upper cut-off is infinite since the longest relaxation time diverges to infinity at the LST. Parameters $S, n$, and the lower cross-over, $\lambda_{0}$, depend on the material structure at the transition.

Beyond the LST, $p>p_{\mathrm{c}}$, the material is a solid. The solid state manifests itself in a finite value of the relaxation modulus at long times, the so-called equilibrium modulus

$$
G_{\mathrm{e}}=\lim _{t \rightarrow \infty} G(t)
$$

Stresses cannot relax completely any more. The upper curves in Fig. 2 show this curving to the right, where at long times an equilibrium stress level will eventually be reached. More data at longer times would be required in order to clearly identify the value of $G_{\mathrm{e}}$. However, $G_{\mathrm{e}}$ can be estimated from the curves, 
and it can be seen that $G_{\mathrm{e}}$ is zero at the gel point and grows with the extent of reaction, $p$.

Rubbery materials beyond the gel point have been studied extensively. A long time ago, Thirion and Chasset [9] recognized that the relaxation pattern of a stress $\tau$ under static conditions can be approximated by the superposition of a power law region and a constant limiting stress $\tau_{1}$ at infinite time:

$$
\tau=\tau_{1}\left(1+\left(\frac{t}{\lambda_{0}}\right)^{-n}\right)
$$

where $\lambda_{0}$ is a material-dependent time constant. They found very low values for the slope $n$ in the power law region, $0.12<n<0.17$.

\subsection{Relaxation Time Spectrum}

The linear viscoelastic behavior of liquid and solid materials in general is often defined by the relaxation time spectrum $H(\lambda)[10]$, which will be abbreviated as 'spectrum' in the following. The transient part of the relaxation modulus as used above is the Laplace transform of the relaxation time spectrum $H(\lambda)$

$$
G(t)=G_{\mathrm{e}}+\int_{0}^{\lambda_{\max }} H(\lambda) \mathrm{e}^{-t / \lambda} \frac{\mathrm{d} \lambda}{\lambda} .
$$

The spectrum is a non-negative function [11] which exists in the range of relaxation times $0<\lambda \leqslant \lambda_{\max }$. An important material property is the longest relaxation time, $\lambda_{\max }$, beyond which the spectrum is equal to zero; $H(\lambda)=0$ for $\lambda>\lambda_{\max }$. The spectrum cannot be measured directly. However, many methods have been proposed to somehow extract $H(\lambda)$ from linear viscoelastic material functions as measured in the appropriate experiments. A comprehensive review of some of those methods was recently presented by Orbey and Dealy [12].

We assume that the spectrum $H(\lambda)$ gradually evolves as the material undergoes transition. There exists a spectrum for the material directly at the transition point, the critical gel. Its characteristic features are twofold: a longest relaxation time (upper limit of the integral) that diverges, $\lambda_{\max } \rightarrow \infty$, and a power law distribution with a negative exponent, $-n$. Both properties are expressed in the self-similar CW spectrum which Chambon and Winter [6,7] found when analyzing chemical gelation experiments (Fig. 3):

$$
H(\lambda)=\frac{S}{\Gamma(n)} \lambda^{-n} \text { for } \lambda_{0}<\lambda<\infty
$$

where $\Gamma(n)$ is the gamma function. Stress relaxation is the same at all scales of observation for such 'self-similar' or 'scale invariant' behavior. It is interesting to note that the critical gel does not have a characteristic time constant, which is a rather unusual property for a viscoelastic material.

The relaxation exponent $n$ is restricted to values between 0 and 1 . The case of $n=0$ corresponds to the limiting behavior of a Hookean solid (the relaxation 


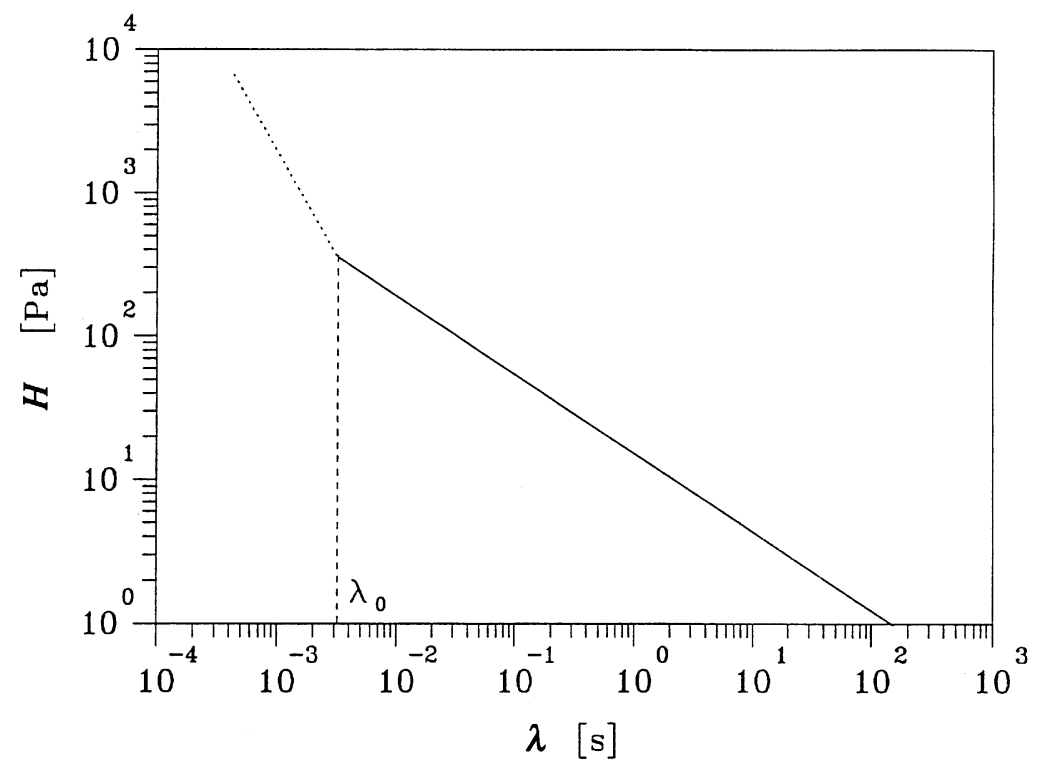

Fig. 3. Schematic of Chambon-Winter gel spectrum. The longest relaxation time diverges to infinity. The relaxation time $\lambda_{0}$ marks the crossover to the short-time behavior, which depends on the material. The depicted case corresponds to a low-molecular-weight precursor (crossover to glass transition region)

modulus is a constant). The restriction of $n$ to values less than unity is necessary to assure a diverging zero-shear viscosity at the gel point.

The self-similar spectrum is not valid at short times, $\lambda<\lambda_{0}$, where the details of chemical structure become important (glass transition, entanglements, etc.). The cross-over to the glass transition at short times is typical for all polymeric materials, for both liquids and solids. The critical gel is no exception in that respect. $\lambda_{0}$ could be used as a characteristic time in the $\mathrm{CW}$ spectrum since it somehow characterizes the molecular building block of the critical gel; however, it has no direct relation to the LST. At times shorter than $\lambda_{0}$, the LST has no immediate effect on the rheology. Indirect effects might be seen as a shift in the glass transition, for instance, but these will not be studied here.

\subsection{Divergence of Longest Relaxation Time}

In the close vicinity of the gel point, $\left|p_{\mathrm{c}}-p\right| \ll 1$, the longest relaxation time diverges in a power law on both sides of the gel point (Fig. 4)

$$
\lambda_{\text {max }} \propto \begin{cases}\left(p_{\mathrm{c}}-p\right)^{-\alpha_{-}} & \text {for sol, } p<p_{\mathrm{c}} \\ \left(p-p_{\mathrm{c}}\right)^{-\alpha_{+}} & \text {for gel, } p>p_{\mathrm{c}} .\end{cases}
$$




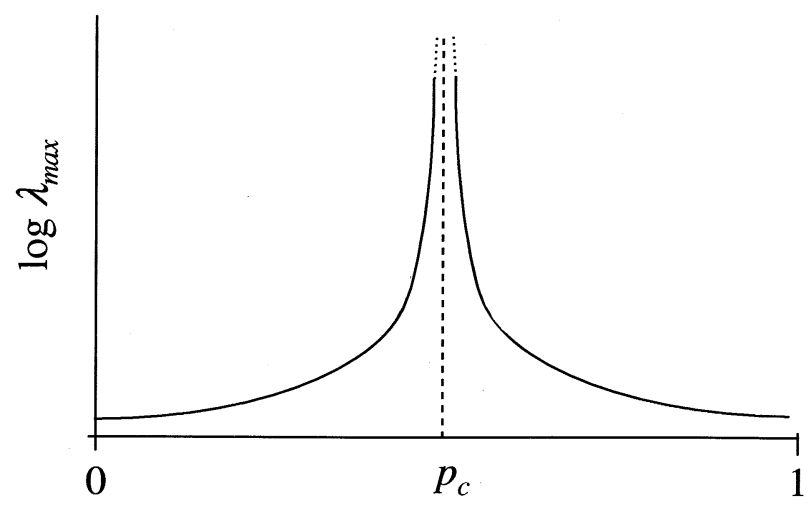

Fig. 4. Schematic of the divergence of the longest relaxation time as the liquid-solid transition is approached from either side

$\alpha_{-}$and $\alpha_{+}$are the critical exponents for the sol and the gel. In the sol, $\lambda_{\max }$ belongs to the largest cluster. The largest cluster reaches infinite size at the gel point, but it still can relax, and the corresponding $\lambda_{\max }$ has become infinitely large. Beyond the gel point, the relaxable components (for chemical gelation this would be the sol fraction, unattached chain ends, long loops, etc.) gradually incorporate into the permanent network, and $\lambda_{\max }$ decays again.

With increasing distance from the gel point, the simplicity of the critical state will be lost gradually. However, there is a region near the gel point in which the spectrum still is very closely related to the spectrum at the gel point itself, $H\left(\lambda, p_{\mathrm{c}}\right)$. The most important difference is the finite longest relaxation time which cuts off the spectrum. Specific cut-off functions have been proposed by Martin et al. [13] for the spectrum and by Martin et al. [13], Friedrich et al. [14], and Adolf and Martin [15] for the relaxation function $G\left(t, p_{\mathrm{c}}\right)$. Sufficiently close to the gel point, $\left|p-p_{\mathrm{c}}\right| \ll 1$, the specific cut-off function of the spectrum is of minor importance. The problem becomes interesting further away from the gel point. More experimental data are needed for testing these relations.

It was a most interesting discovery that not only the longest relaxation time diverges at LST, but that all the shorter relaxation modes show a very distinct pattern. The longest mode escapes the measurement near LST while the spectrum of the shorter modes is still accessible. The intent of this study is to explore the occurrence of this relaxation time spectrum in a broad range of solidifying materials and in a time or frequency window which is as wide as possible. The properties of the self-similar CW spectrum, Eq. 1-5, will be mapped out in Sect. 3. The behavior at LST then will serve as a reference state for the analysis of rheological phenomena in the vicinity of LST. This will set the stage for reviewing experimental data from several laboratories. Observations on chemical gelation will guide the analysis of various types of LST. The possibility will 
be suggested that there exists a universal framework for many LSTs of different origin. In that spirit, the terms 'liquid-solid transition' (LST) and 'gel point' will be used synonymously.

\subsection{Interrelation Between Critical Exponents}

Steady shear flow properties are sensitive indicators of the approaching gel point for the liquid near LST, $p<p_{\mathrm{c}}$. The zero shear viscosity $\eta_{0}$ and equilibrium modulus $G_{\mathrm{e}}$ grow with power laws [16]

$$
\begin{array}{ll}
\eta_{0} \propto\left(p_{\mathrm{c}}-p\right)^{-s} & \text { for sol, } p<p_{\mathrm{c}} \\
G_{\mathrm{e}} \propto\left(p-p_{\mathrm{c}}\right)^{z} & \text { for gel, } p>p_{\mathrm{c}}
\end{array}
$$

having critical exponents, $s$ and $z$. The viscosity of the sol increases due to the diverging cluster size. The equilibrium modulus of the gel gradually builds up since an increasing fraction of the molecules join, and thereby strengthen the sample spanning permanent network (Fig. 5).

As a result, we find for sols that the divergence of the above zero shear viscosity $\eta_{0}$ and of two other linear viscoelastic material functions, first normal stress coefficient $\psi_{1}$ and equilibrium compliance $J_{\mathrm{e}}^{0}$, depends on the divergence of $\lambda_{\max }[17]$

$$
\lambda_{\max }(p) \propto \eta_{0}^{1 /(1-n)} \propto \psi_{1}^{1 /(2-n)} \propto J_{\mathrm{e}}^{0^{1 / n}} \text { for sol, } p<p_{\mathrm{c}} .
$$

Only the value of the relaxation exponent is needed. The critical exponent $\alpha_{-}$of the longest relaxation time (compare Eqs. 1-6 and 1-7) is therefore on an equal footing with the critical exponent of the viscosity:

$$
s=(1-n) \alpha .
$$

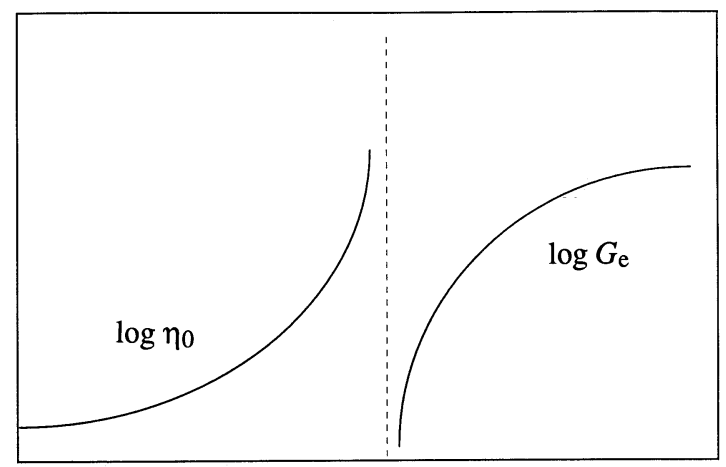

0 $p_{\mathrm{c}}$
Fig. 5. Schematic of the divergence of zero-shear viscosity, $\eta_{0}$, and equilibrium modulus, $G_{\mathrm{e}}$. The LST is marked by $p_{\mathrm{c}}$ 
For the relaxation of the solid near the gel point, the critical gel may serve as a reference state. The long time asymptote of $G(t)$ of the nearly critical gel, the equilibrium modulus $G_{\mathrm{e}}$, intersects the $G(t)=S t^{-n}$ of the critical gel at a characteristic time (Fig. 6) which we will define as the longest relaxation time of the nearly critical gel [18]

$$
G_{\mathrm{e}}=\left(S t^{-n}\right)_{t=\lambda_{\max }} \rightarrow \lambda_{\max }=\left(\frac{G_{\mathrm{e}}(p)}{S}\right)^{-1 / n} \text { for gel, } p>p_{\mathrm{c}} .
$$

It obeys the typical characteristics, namely the divergence to infinity as $G_{\mathrm{e}}$ goes to zero (gel point) and the approach of a zero value as $G_{\mathrm{e}}$ becomes large. Again, only the relaxation exponent $n$ is needed for relating the divergence of $\lambda_{\text {max }}$ with that of $G_{\mathrm{e}}$; compare Eqs. 1-8 and 1-11:

$$
z=n \alpha_{+} \text {for gel, } p>p_{\mathrm{c}} .
$$

The exponents $\alpha_{-}$and $\alpha_{+}$depend not only on the relaxation exponent $n$, but also on the dynamic exponents $s$ and $z$ for the steady shear viscosity of the sol and the equilibrium modulus of the gel.

The analysis may be simplified by postulating symmetry of the diverging $\lambda_{\max }$ on both sides of the gel point [18]. A power law exponent (see Eq. 1-6) which is the same on both sides,

$$
\alpha=\alpha_{-}=\alpha_{+}
$$

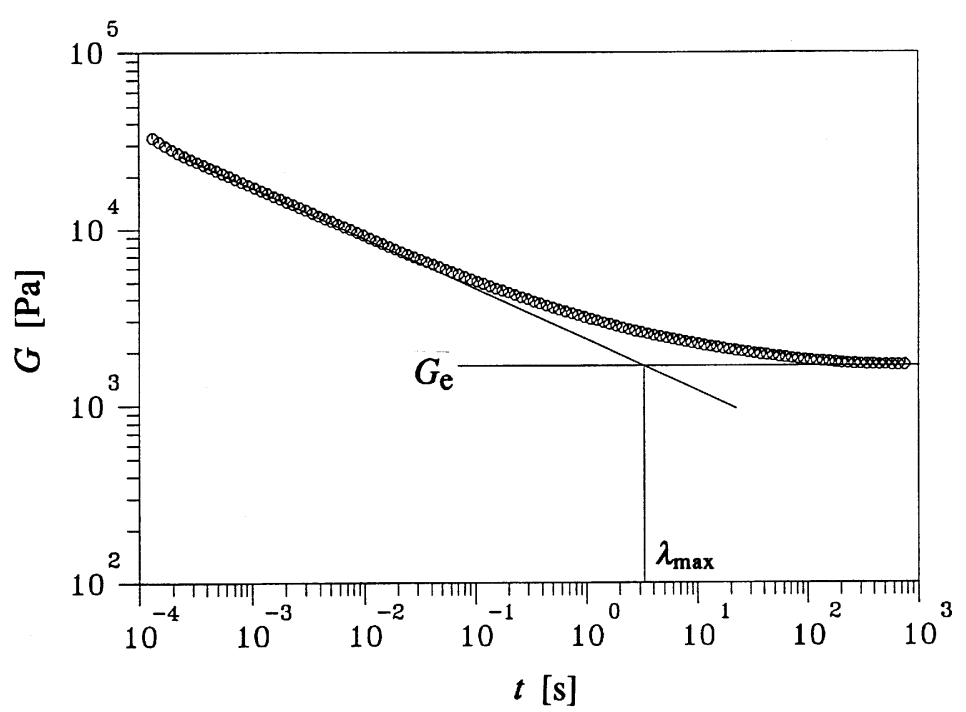

Fig. 6. Evaluation of the longest relaxation time for a sample beyond the gel point, $p>p_{\mathrm{c}}$ : intersect of horizontal line for $G_{\mathrm{e}}$ with the power law of the critical gel, $S t^{-n}$ 
leads to the interesting relations between critical exponents $[13,18,19]$

$$
\begin{aligned}
& n=z /(z+s) . \\
& \alpha=s+z, \\
& s=(1-n) \alpha ; z=n \alpha .
\end{aligned}
$$

Only two of the exponents ( $\alpha$ and $n$, for instance) are sufficient to describe the rheology of nearly critical gels. The front factor is more difficult to estimate, but it most likely differs on both sides.

These relations will be useful for testing theories, since, except for the symmetry hypothesis, no specific assumptions were introduced in the derivation. Theory might give an answer about the validity of the above symmetry hypothesis. In fact, the theory of Goldbart and Goldenfeld [20] that is based on statistical mechanics yields Eq. 1-14. However, there does not seem to be an easy way of proving or disproving this hypothesis at this time. The wide range of values for the relaxation exponent, $0<n<1$, lets us expect that the dynamic exponents $s$ and $z$ are non-universal as well.

\section{Theory of Gelation}

This study is mostly concerned with experimental aspects, especially since a quantitative prediction of the self-similar spectrum (value of critical exponent and prefactor) from first principles seems to be still lacking, although several theories predict the evolution of cluster growth during gelation. Excellent reviews of theory have been given by Stauffer et al. [3,16] and Vilgis [21]. We refer to these for a deeper study and only highlight several of the theoretical predictions in the following.

\subsection{Branching Theories}

Branching models are based on multifunctional molecules of different types between which covalent bonds are formed to yield a network structure. One of the multifunctional molecules is required to carry at least three functional groups, while the other one can have two functional groups. The overall extent of reaction, $p$, equals the a priori probability that any given functional group has condensed. The earliest of these branching theories was developed by Flory $[1,22]$ and Stockmayer [2]. Using combinatorial approaches, they derived an expression for the molecular weight distribution, and subsequently the critical extent of reaction, $p_{\mathrm{c}}$, at which the molecular weight diverges, $M_{\mathrm{w}} \rightarrow \infty$ (gel point). Their approach includes several simplifying assumptions which are 
usually not valid in real systems, i.e. (1) the reactivities of all functional groups of the same type are equal and independent of each other, (2) no intramolecular reactions between functional groups on the same cluster ('loop formation') are allowed, (3) the crosslinks are randomly formed between any pair of functional groups that can form a bond, and (4) point-like monomers are assumed (no steric hindrance and excluded volume effects). More advanced branching models were developed later. The two most widely used of these are the so-called recursive theory $[23,24]$ and the cascade theory [25-28]. These later models can deal approximately with nonidealities such as cyclization and long-range substitution effects. All branching theories are mean field theories and yield the same simple expression for the critical extent of reaction (for the same chemical model) depending on the geometry of the network. Special cases are:

Case 1. Homopolymerization of similar $f$-functional molecules:

$$
p_{\mathrm{c}}=\frac{1}{\bar{f}_{2}-1}
$$

The same relation is found for the end-linking of molecules of low functionality ( $f=3$ or 4$)$ and for the vulcanization of long molecular chains. The secondmoment average number of cross-linking sites along the chain, $\bar{f}_{2}$, is defined as

$$
\bar{f}_{2}=\frac{\sum_{f} f^{2} n_{f}}{\sum_{f} f n_{f}}
$$

with $n_{f}=$ number of molecules of functionality $f$.

Case 2. Cross-linking of $f$-functional molecules $\mathrm{A}_{f}$ with $g$-functional molecules $\mathrm{B}_{g}$, which are mixed at a molar ratio $r=\sum_{f} f n_{\mathrm{A} f} / \sum_{g} g n_{\mathrm{B} g}$

$$
p_{\mathrm{A}, c}=\frac{1}{\sqrt{r\left(\bar{f}_{2}-1\right)\left(\bar{g}_{2}-1\right)}}
$$

with $p_{\mathrm{B}}=r p_{\mathrm{A}}$. The stoichiometric ratio of a sample must be chosen between a lower and upper critical value

$$
r_{1}=\frac{1}{\left(\bar{f}_{2}-1\right)\left(\bar{g}_{2}-1\right)} ; \quad r_{\mathrm{u}}=\frac{1}{r_{1}}
$$

otherwise the reaction stops before reaching the gel point. The relations in Eq. 2-4 follow from Eq. 2-3 when considering species $\mathrm{A}_{f}$ or species $\mathrm{B}_{g}$ fully reacted, respectively.

Experimental results of $p_{\mathrm{c}}, r_{1}$, and $r_{\mathrm{u}}$ were found to agree reasonably well with these predictions despite the inherent assumptions [29-31]. 


\subsection{Percolation Theory}

Percolation theory describes [32] the random growth of molecular clusters on a $d$-dimensional lattice. It was suggested to possibly give a better description of gelation than the classical statistical methods (which in fact are equivalent to percolation on a Bethe lattice or Caley tree, Fig. 7a) since the mean-field assumptions (unlimited mobility and accessibility of all groups) are avoided $[16,33]$. In contrast, immobility of all clusters is implied, which is unrealistic because of the translational diffusion of small clusters. An important fundamental feature of percolation is the existence of a critical value $p_{\mathrm{c}}$ of $p$ (bond formation probability in random bond percolation) beyond which the probability of finding a percolating cluster, i.e. a cluster which spans the whole sample, is non-zero.

In random bond percolation, which is most widely used to describe gelation, monomers, occupy sites of a periodic lattice. The network formation is simulated by the formation of bonds (with a certain probability, $p$ ) between nearest neighbors of lattice sites, Fig. 7b. Since these bonds are randomly placed between the lattice nodes, intramolecular reactions are allowed. Other types of percolation are, for example, random site percolation (sites on a regular lattice are randomly occupied with a probability $p$ ) or 'random random' percolation (also known as continuum percolation: the sites do not form a periodic lattice but are distributed randomly throughout the percolation space). While the

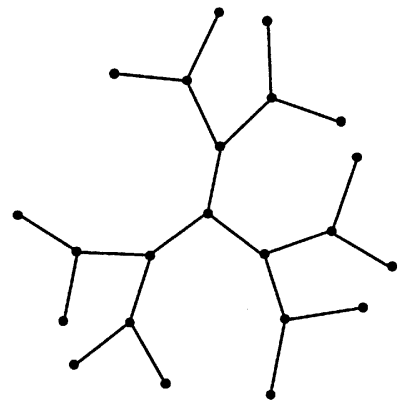

$\mathbf{a}$

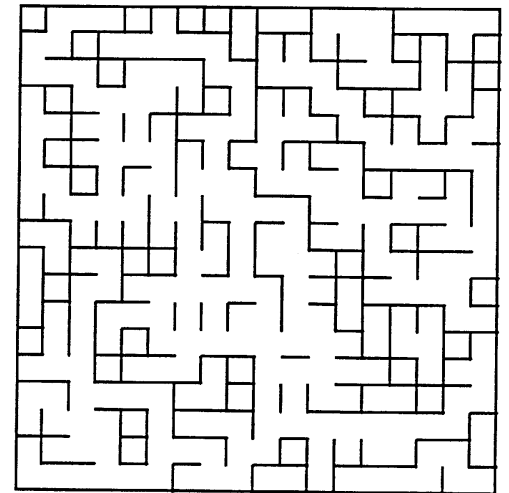

b

Fig. 7. Comparison of a the structure of the Bethe lattice with a functionality of 3 (only part of the system is shown) and $\mathbf{b}$ a two-dimensional square lattice [16]. For the Bethe lattice, each possible bond is shown as a line connecting two monomers. In FS theory an actual bond of these possible bonds is formed with probability $p$. For the square lattice, each bond that has been formed is shown as a short line connecting two monomers, while the monomers are not shown 
random site percolation is not directly relevant to gelation [16], continuum percolation is of particular value, since in real systems the cluster-forming molecules are not distributed regularly in space.

In general, percolation is one of the principal tools to analyze disordered media. It has been used extensively to study, for example, random electrical networks, diffusion in disordered media, or phase transitions. Percolation models usually require approximate solution methods such as Monte Carlo simulations, series expansions, and phenomenological renormalization [16]. While some exact results are known (for the Bethe lattice, for instance), they are very rare because of the complexity of the problem. Monte Carlo simulations are very versatile but lack the accuracy of the other methods. The above solution methods were employed in determining the critical exponents given in the following section.

\subsection{Scaling Near the LST}

All theories yield unique scaling relationships for molecular (e.g. mean cluster size, size distribution) and bulk properties (e.g. equilibrium modulus) near the critical point, but critical exponent values and relations between different critical exponents are different. This scaling is common for material behavior near any critical point, i.e. the polymeric material near the gel point exhibits a behavior analogous, for example, to a fluid near its vapor-liquid critical point. For the critical gel, weight average molecular weight $M_{\mathrm{w}}$, typical cluster size $R_{\text {char }}$, and gel fraction $f_{\mathrm{g}}$ scale similarly with $\left|p-p_{\mathrm{c}}\right|$ as the inverse of the derivative of osmotic pressure with respect to concentration $(\partial \pi / \partial c)^{-1}$, correlation length $\xi$, and concentration fluctuations $\Delta c$, respectively, scale with $T-T_{\mathrm{c}}$ in case of a fluid at the vapor-liquid critical point $[3,34]$. The following scaling relationships for these static properties are commonly found in the literature $[16,35]$ :

$$
\begin{array}{ll}
M_{\mathrm{w}} \propto\left|p-p_{\mathrm{c}}\right|^{-\gamma} & p<p_{\mathrm{c}} \\
R_{\mathrm{char}} \propto\left|p-p_{\mathrm{c}}\right|^{-v} & p<p_{\mathrm{c}} \\
f_{\mathrm{g}} \propto\left|p-p_{\mathrm{c}}\right|^{\beta} & p>p_{\mathrm{c}}
\end{array}
$$

The cluster mass distribution at the gel point scales with the molecular weight of those clusters

$$
N(M) \propto M^{-\tau} \quad p=p_{\mathrm{c}}
$$

To describe the cluster mass distribution in the vicinity of the gel point, a cut-off function $f\left(M / M_{\max }\right)$ is introduced [36] (Fig. 8)

$$
N(M) \propto M^{-\tau} f\left(\frac{M}{M_{\max }}\right)
$$




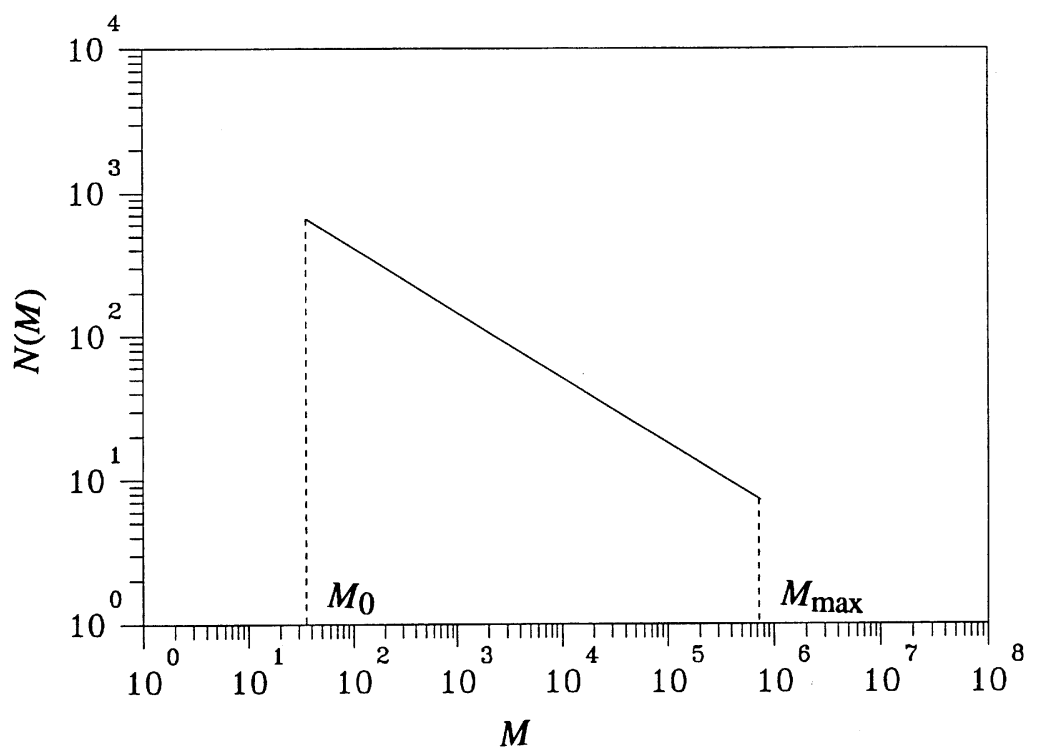

Fig. 8. Power law molecular weight distribution in the vicinity of the LST. $M_{0}$ is the molecular weight of the smallest precursor molecule, and $M_{\max }$ the molecular weight of the largest cluster present in the polymer

with

$$
M_{\max } \propto\left|p-p_{\mathrm{c}}\right|^{-1 / \sigma}
$$

Percolation theory predictions for these critical exponents are $\gamma=1.76$, $v=0.89, \beta=0.39, \tau=2.2$ and $\sigma=0.46$. The Flory-Stockmayer theory also predicts this scaling behavior near the gel point, with exponents $\gamma=1, v=0.5$, $\beta=1, \tau=2.5$, and $\sigma=0.5[3,37]$.

Colby et al. [35] proposed an interesting experimental approach to measure the static exponents. They noticed that it is hard to accurately measure the chemical extent of reaction, $p$, and thus eliminated this variable (more precisely the distance from the gel point $\left.\left|p-p_{\mathrm{c}}\right|\right)$ from the scaling relations. For example combining Eqs. 2-5 and 2-6 yields the following relation between the weight average molecular weight, $M_{\mathrm{w}}$, and the characteristic radius, $R_{\mathrm{char}}$ :

$$
M_{\mathrm{w}} \propto R_{\mathrm{char}}^{\gamma / v}
$$

Similar relations between different scaling exponents were also developed by Stauffer [37] by combining two of the scaling relations at a time to eliminate $\left|p-p_{\mathrm{c}}\right|$.

Besides the static scaling relations, scaling of dynamic properties such as viscosity $\eta$ and equilibrium modulus $G_{\mathrm{e}}[16,34]$, see Eqs. 1-7 and 1-8, is also predicted. The equilibrium modulus can be extrapolated from dynamic experiments, but it actually is a static property [38]. 
The scaling of the relaxation modulus $G(t)$ with time (Eq. 1-1) at the LST was first detected experimentally [5-7]. Subsequently, dynamic scaling based on percolation theory used the relation between diffusion coefficient and longest relaxation time of a single cluster to calculate a relaxation time spectrum for the sum of all clusters [39]. This resulted in the same scaling relation for $G(t)$ with an exponent $n$ following Eq. 1-14.

It is interesting to note here that the cluster mass distribution and the relaxation modulus $G(t)$ at the LST scale with cluster mass and with time, respectively, while all other variables (dynamic and static) scale with the distance from $p_{\mathrm{c}}$ in the vicinity of the gel point.

The classical theory predicts values for the dynamic exponents of $s=0$ and $z=3$. Since $s=0$, the viscosity diverges at most logarithmically at the gel point. Using Eq. 1-14, a relaxation exponent of $n=1$ can be attributed to classical theory [34]. Dynamic scaling based on percolation theory $[34,40]$ does not yield unique results for the dynamic exponents as it does for the static exponents. Several models can be found that result in different values for $n, s$ and $z$. These models use either Rouse and Zimm limits of hydrodynamic interactions or Electrical Network analogies. The following values were reported [34, 39]: (Rouse, no hydrodynamic interactions) $n=0.66, s=1.35$, and $z=2.7$, (Zimm, hydrodynamic interactions accounted for) $n=1, s=0$, and $z=2.7$, and (Electrical Network) $n=0.71, s=0.75$ and $z=1.94$.

De Gennes [41] predicted that percolation theory should hold for crosslinking of small molecule precursors. However, he argued that for vulcanizing polymers (high $M_{\mathrm{w}}$ ), only a very narrow regime near the gel point exists for which percolation is valid, i.e. these polymers should exhibit more mean fieldlike behavior.

\subsection{Critical Gel as Fractal Structure}

Based on the fractal behavior of the critical gel, which expresses itself in the self-similar relaxation, several different relationships between the critical exponent $n$ and the fractal dimension $d_{\mathrm{f}}$ have been proposed recently. The fractal dimension $d_{\mathrm{f}}$ of the polymer cluster is commonly defined by $[16,42]$

$$
R \propto M^{1 / d_{\mathrm{f}}}
$$

where $R$ is the radius of gyration. Assuming hyperscaling $d_{\mathrm{f}}$ can be related to the critical exponents by (not valid for mean field theories) $[16,34]$

$$
d_{\mathrm{f}}=d-\frac{\beta}{v}
$$

This results in a value of $d_{\mathrm{f}}=2.5$ for bond percolation on a 3-dimensional lattice. The fractal dimension of the Bethe lattice (Flory-Stockmayer theory) is 
$d_{\mathrm{f}}=4[16]$, which leads to a physical discrepancy, since any value higher than 3 (dimensionality of the sample) results in the cluster density increasing with cluster size $\left(\rho \propto R^{d_{\mathrm{f}}-d}\right)$. For $d=6$ the hyperscaling assumption and mean field theories are compatible, i.e. Eq. 2-13 gives the correct fractal dimension for the classical theory.

Muthukumar and Winter [42] investigated the behavior of monodisperse polymeric fractals following Rouse chain dynamics, i.e. Gaussian chains (excluded volume fully screened) with fully screened hydrodynamic interactions. They predicted that $n$ and $\bar{d}_{\mathrm{f}}$ (the fractal dimension of the polymer if the excluded volume effect is fully screened) are related by

$$
n=\frac{\bar{d}_{\mathrm{f}}}{\bar{d}_{\mathrm{f}}+2}
$$

Hess et al. [43] extended this approach to monodisperse chains with excluded volume effects (swollen clusters). They realized that, although the linkage process can be described by percolation, bond percolation does not give a correct picture of crosslinking between long chains because these chains are flexible, whereas bond percolation theory is based on stiff bonds. Thus, even though the connectivity of the critical gel may be prescribed by bond percolation theory, the dynamic properties of the object are drastically affected by the replacement of rigid bonds by flexible chains. Their investigation resulted in the same functional dependence of $n$ and $d_{\mathrm{f}}$ (the fractal dimension when excluded volume effects are taken into account):

$$
n=\frac{d_{\mathrm{f}}}{d_{\mathrm{f}}+2}
$$

where $d_{\mathrm{f}}$ and $\bar{d}_{\mathrm{f}}$ are related by

$$
\bar{d}_{\mathrm{f}}=\frac{2 d_{\mathrm{f}}}{d+2-2 d_{\mathrm{f}}}
$$

Muthukumar [44] further investigated the effects of polydispersity, which are important for crosslinking systems. He used a hyperscaling relation from percolation theory to obtain his results. If the excluded volume is not screened, $n$ is related to $d_{\mathrm{f}}$ by

$$
n=\frac{d_{\mathrm{f}}}{d_{\mathrm{f}}+2}
$$

In the case of full screening of excluded volume he obtained

$$
n=\frac{d}{\bar{d}_{\mathrm{f}}+2}=\frac{d\left(d+2-2 d_{\mathrm{f}}\right)}{2\left(d+2-d_{\mathrm{f}}\right)}
$$

Especially in the latter case, a small change in the fractal dimension can lead to a significant change in $n$, and he therefore concluded that $n$ can take values between 0 and 1 (for $d_{\mathrm{f}}$ ranging from 2.5 to 1.25 , see Fig. 9). 


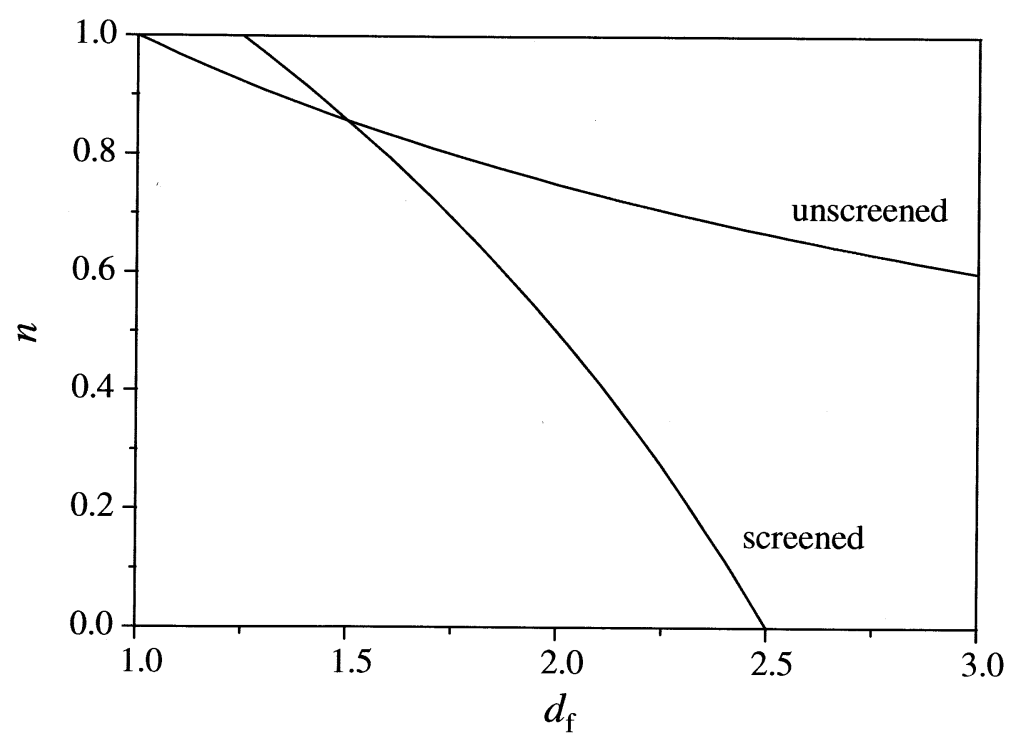

Fig. 9. Relation between relaxation exponent $n$ and fractal dimension $d_{\mathrm{f}}$ for a three-dimensional network. In case of complete screening of excluded volume, values of $0<n<1$ are possible if $d_{\mathrm{f}}$ is chosen between 1.25 and 2.5

If only partial screening is present, the fractal dimension takes a value somewhere between $d_{\mathrm{f}}$ and $\bar{d}_{\mathrm{f}}$. According to this model, a crosslinker deficiency, which leads to a more open structure and therefore a lower value of $d_{\mathrm{f}}$, increases the value of $n$. Dilution of the precursor with a non-reactive species has the same effect on the relaxation exponent.

\subsection{The Notion of Topology}

Goldbart and Goldenfeld [20] challenged the notion that gelation could be described in terms of the purely geometrical description of percolation theory. They developed a theory based on statistical mechanics arguments. The crosslinking system cannot be uniquely specified by the positions of crosslinks only, as is done in percolation theory. Topology needs to be considered as well. They argue that the condition for the liquid-solid transition is a sufficiently complex topology rather than a sufficient degree of connectivity, in contrast to percolation, which does not take the contribution of trapped entanglements into account. They define the solid state in terms of a non-vanishing shear modulus (as $t \rightarrow \infty$ ) and in terms of breaking of the translational invariance of the Hamiltonian. This implies that rigidity of the solid state is due to the preference of atoms to localize close to certain neighbors in order to minimize the free energy of the system rather than due to long range forces, i.e. rigidity is 
a consequence of thermodynamics. Percolation models do not address the question of how rigidity emerges. The new model is a microscopic theory of the liquid-solid transition based on a physical model of flexible chains and solvent. The transition to the solid state as the crosslink density increases beyond the critical density is a continuous one, and hence is a second order transition. This is rather unusual for liquid-solid transitions, which are usually first order. The second order transition is accompanied by scale invariance, and therefore implies scaling behavior of the shear modulus as detected by Chambon and Winter [5], but it does so only at the critical point. Goldenfeld and Goldbart [20] developed relations between scaling exponents $z$, $s$, and $n$ for the equilibrium modulus $G_{\mathrm{e}}$, the zero-shear viscosity $\eta_{0}$, and the complex modulus $G(t)$, respectively, and predict Eq. 1-14, $n=z /(s+z)$, from some general arguments. The exponent of the equilibrium modulus is predicted to equal the scaling exponent for the correlation length $\xi$. However, the theory does not predict any values for these scaling exponents, which makes comparison with experimental data difficult.

\subsection{Kinetic Theory (Smoluchowski Equation)}

All models described up to here belong to the class of equilibrium theories. They have the advantage of providing structural information on the material during the liquid-solid transition. Kinetic theories based on Smoluchowski's coagulation equation [45] have recently been applied more and more to describe the kinetics of gelation. The Smoluchowski equation describes the time evolution of the cluster size distribution $N(k)$ :

$$
\frac{d N(k)}{d t}=\frac{1}{2} \sum_{i+j=k} K_{i j} N(i) N(j)-N(k) \sum_{j=1}^{\infty} K_{k j} N(j)
$$

$N(k)$ denotes the number of clusters of size $k$ (i.e. $k$-mers), and $K_{i j}$ is the reaction kernel that gives the probability of a cluster of size $i$ reacting with one of size $j$. The first sum accounts for coalescence of clusters of size $i$ and $(k-i)$ to give a cluster of size $k$, while the second sum accounts for the loss of clusters of size $k$ due to binary collisions with other clusters. The Smoluchowski equation is able to describe and distinguish between gelling and non-gelling systems. In the former, the mean cluster size diverges as $t$ approaches the gel point $t_{\mathrm{c}}$; in the latter it keeps increasing with time. Although the equation was originally developed only for irreversible coagulation, it can be easily extended to reversible coagulation by adding fragmentation kernels $F_{i j}$ to describe the unimolecular fragmentation process [46]:

$$
\begin{aligned}
\frac{d N(k)}{d t}= & \frac{1}{2} \sum_{i+j=k}\left\{K_{i j} N(i) N(j)-F_{i j} N(k)\right\} \\
& -\sum_{j=1}^{\infty}\left\{K_{k j} N(k) N(j)-F_{k j} N(k+j)\right\}
\end{aligned}
$$


where $F_{i j}$ describes the probability of a $(i+j)$-mer to break up and form and $i$-mer and a $j$-mer. Both Equations 2-19 and 2-20 constitute infinite sets of coupled non-linear equations which have to be solved for a given initial cluster size distribution $N\left(k, t_{0}\right)$.

The difficulty is now to determine the functional form of the reaction and fragmentation kernels, $K_{i j}$ and $F_{i j}$. The specific form is determined by the coagulation mechanism. For gelation processes, usually only the reaction kernel $K_{i j}$ is considered, i.e. the process is viewed as being irreversible. A variety of kernels for coagulation processes can be found in the literature [47]. Most of these kernels are homogeneous functions of $i$ and $j$, at least for large $i$ and $j$. Ernst [47] and Van Dongen and Ernst [48] used two exponents $\mu$ and $v$ to describe the $i$ and $j$ dependence of $K_{i j}=K(i, j)$ :

$$
\begin{aligned}
& K(a i, a j)=a^{\Lambda} K(i, j)=a^{\Lambda} K(j, i) \\
& K(i, j) \propto i^{\mu} j^{v}
\end{aligned}
$$

with $j \gg 1$ and $\lambda=\mu+v$. Two physical restrictions exist on the exponents $\mu$ and $v$, because the reaction rate cannot increase faster than the cluster size: $\Lambda=\mu+v \leqslant 2$ and $v \leqslant 1 . \Lambda$ characterizes the reaction rate of two large interpenetrable clusters, i.e. $K(j, j) \propto j^{\mu+v}$, and $v$ describes the reaction of a large cluster with a very small cluster, i.e. $K(1, j) \propto j^{v}$. Furthermore, $\Lambda$ also decides whether the Smoluchowski equation describes aggregation or gelation, i.e. the formation of an infinite cluster in finite time only occurs if $\Lambda>1$.

Three growth classes are usually distinguished. These are class I with $\mu>0$, class II with $\mu=0$ and class III with $\mu<0$. In the case of class I, growth interactions between two large clusters are dominant. Class I growth can describe both aggregating and gelling systems. For gelation, $v \leqslant 1<\Lambda \leqslant 2$. The cluster distribution decays as $N(k) \propto k^{-\tau}$ with $\tau=\frac{1}{2}(\Lambda+3)>2$. Also, near the gel point, the weight average mass defined as $M_{\mathrm{w}}=\sum k^{2} N(k) / \sum k N(k)$ diverges as $M_{\mathrm{w}} \propto\left|t-t_{\mathrm{c}}\right|^{-\gamma}$ with $\gamma=(3-\Lambda) /(\Lambda-1)$, the typical cluster mass (the $z$-average mass, $\left.M_{\mathrm{z}}=\sum k^{3} N(k) / \sum k^{2} N(k)\right)$ diverges as $M_{\mathrm{z}} \propto\left|t-t_{\mathrm{c}}\right|^{-1 / \sigma}$ with $\sigma=(\Lambda-1) / 2$, and the gel fraction diverges as $f_{\mathrm{g}} \propto\left|t-t_{\mathrm{c}}\right|^{\beta}$ with $\beta=1$ [49]. These scaling equations correspond to Eqs. 2-8, 2-5, 2-10, and 2-7, respectively, as presented earlier in this Section. If one uses the Flory-Stockmayer gelation theory, the reaction kernel equals $K_{i j}=i j$, since all sites on a cluster are assumed to be equally reactive [50]. This is a typical example of a homogeneous kernel which gives class I growth. The exponents are therefore $\mu=1$ and $v=1$, resulting in $\Lambda=2$. This results in scaling exponents $\tau=2.5, \gamma=1, \sigma=0.5$, and $\beta=1$, which are also predicted by the FS-theory (see section 2.1.).

In class II growth, the large-large (class I) and small-large (class III) interactions are equal. Since $\mu=0$, it follows that $v=\Lambda$. Because of the restriction on $v(v \leqslant 1)$ and the requirement of $\Lambda>1$ for gelation this class can only describe non-gelling growth. Interactions between small and large clusters govern class III growth. From $\mu<0$, it follows that $v>\Lambda$, i.e. class III growth is defined by $\Lambda<v \leqslant 1$. Like class II growth, it can only describe aggregation. 


\subsection{Computer Simulations}

Alternatively, Leung and Eichinger [51] proposed a computer simulation approach which does not assume any lattice as the classical and percolation theory. Their simulations are more realistic than lattice percolation, since spatially closer groups form bonds first and more distant groups at later stages of network formation. However, the implicitly introduced diffusion control is somewhat obscure. The effects of intramolecular reactions were more realistically quantified, and the results agree quite well with experimental observations $[52,53]$.

\section{Viscoelastic Properties at and around the Liquid-Solid Transition}

\subsection{Linear Viscoelasticity of Liquids and Solids}

The time-dependent rheological behavior of liquids and solids in general is described by the classical framework of linear viscoelasticity $[10,54]$. The stress tensor $\tau$ may be expressed in terms of the relaxation modulus $G(t)$ and the strain history:

$$
\tau(t)=\int_{-\infty}^{t} \frac{\partial G\left(t-t^{\prime}\right)}{\partial t^{\prime}} \mathbf{C}^{-1}\left(t ; t^{\prime}\right) d t^{\prime}
$$

or, alternatively:

$$
\tau(t)=-\int_{-\infty}^{t} G\left(t-t^{\prime}\right) \frac{\partial \mathbf{C}^{-1}\left(t ; t^{\prime}\right)}{\partial t^{\prime}} d t^{\prime} .
$$

The relaxation modulus is often expressed with the relaxation time spectrum, Eq. 1-4:

$$
\tau(t)=G_{\mathrm{e}} \mathbf{C}^{-1}\left(t ; t_{0}\right)+\int_{-\infty}^{t} \int_{0}^{\lambda_{\max }} H(\lambda) \mathrm{e}^{-\left(t-t^{\prime}\right) / \lambda} \frac{\mathrm{d} \lambda}{\lambda^{2}} \mathbf{C}^{-1}\left(t ; t^{\prime}\right) d t^{\prime}
$$

Here we describe the strain history with the Finger strain tensor $\mathbf{C}^{-1}\left(t ; t^{\prime}\right)$ as proposed by Lodge [55] in his rubber-like liquid theory. This equation was found to describe the stress in deforming polymer melts as long as the strains are small (second strain invariant below about 3 [56]). The permanent contribution $G_{\mathrm{e}} \mathbf{C}^{-1}\left(t ; t_{0}\right)$ has to be added for a linear viscoelastic solid only. $\mathbf{C}^{-1}\left(t ; t_{0}\right)$ is the strain between the stress free state $t_{0}$ and the instantaneous state $t$. Other strain measures or a combination of strain tensors, as discussed in detail by Larson [57], might also be appropriate and will be considered in future studies. A combination of Finger $\mathbf{C}^{-1}\left(t ; t^{\prime}\right)$ and Cauchy $\mathbf{C}\left(t ; t^{\prime}\right)$ strain tensors is known to express the finite second normal stress difference in shear, for instance. 
The stress $\tau$ is more difficult to model for a material which is deforming while solidifying. A crosslinking polymer, for instance, constantly introduces new crosslinks even during relaxation processes. Molecules or segments of molecules gradually lose their mobility, since the crosslinking locks them into their relative positions with neighboring molecules. As a starting point, we consider materials for which the crosslinking reaction has been stopped or slowed down so severely that they may be treated as chemically stable during a rheological experiment. This quasi-stability will be explained further in Sect. 6.2.

\subsection{Viscoelastic Material Functions of Critical Gels}

We expect that the classical framework of linear viscoelasticity also applies at the gel point. The relaxation spectrum for the critical gel is known and can be inserted into Eq. 3-3. The resulting constitutive equation will be explored in a separate section (Sect. 4). Here we are mostly concerned about the material parameters which govern the wide variety of critical gels.

The linear viscoelastic behavior of the critical gel, as defined in Eqs. 1-1 and 1-5, depends on two material parameters, the relaxation exponent $n$ and the front factor $S$. Depending on their values, the critical gel is more soft or more stiff. The relaxation exponent strongly depends on molecular and structural details which affect the development of long range connectivity. These are molecular weight of the precursor, stoichiometric ratio, amount and molecular weight of inert diluent in the material, and bulkiness of the crosslinks (filler effect). The relaxation exponent does not have a universal value, as one might expect for a property at a critical point. ${ }^{1}$ The critical gel is generally very soft and fragile when the relaxation exponent is large, $n \rightarrow 1$, and the front factor $S$ is small. Stiff critical gels have a small $n$ value $(n \rightarrow 0)$ and a large $S$. For many systems, the front factor is not independent, but depends on the relaxation exponent

$$
S=G_{0} \lambda_{0}^{n}
$$

where $G_{0}$ and $\lambda_{0}$ are the plateau modulus of the fully crosslinked material and the characteristic time of the precursor molecule (building block of the gel), respectively [58,59]. This ensures a soft gel for $n \rightarrow 1$ and a stiff gel for $n \rightarrow 0$. Figure 10 shows data of $S$ and $n$ measured by Izuka et al. [59] on polycaprolactone critical gels with different stoichiometric ratios. The dashed line connects the modulus of the fully crosslinked material and the zero-shear viscosity of the precursor, which is in the order of $G_{0} \lambda_{0}$.

The gel stiffness, $S$, was also found to depend on the molecular weight of the polymer precursor. For end-linking PDMS, $S$ decreases with increasing

\footnotetext{
${ }^{1}$ Our first two critical gels had an exponent value of $n \approx 0.5$, which made us believe initially that this would be the only possible value
} 


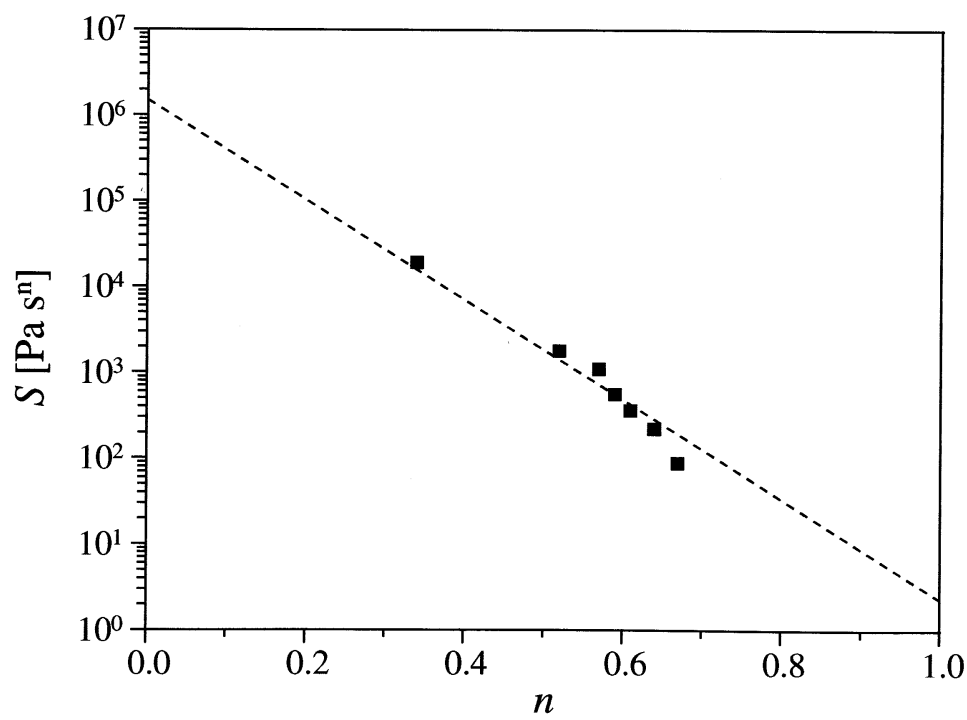

Fig. 10. Experimental values of the gel stiffness $S$ plotted against the relaxation exponent $n$ for crosslinked polycaprolactone at different stoichiometric ratios [59]. The dashed line connects the equilibrium modulus of the fully crosslinked material (on left axis) and the zero shear viscosity of the precursor (on right axis)

$M_{\mathrm{w}}$ because of an increase in strand length between crosslinks [7]. In contrast, vulcanizing polybutadienes of high molecular weight $\left(M_{\mathrm{w}}>\right.$ entanglement molecular weight) show a relaxation exponent of about or somewhat below 0.5 and an increase in gel stiffness with increasing precursor molecular weight. For this study, De Rosa and Winter [60] crosslinked polybutadienes (PBD) with long linear molecules of (nearly) uniform molecular weight and measured the relaxation time spectrum at increasing extents of reaction (Fig. 11 depicts the critical gel spectrum for the PBD with a molecular weight of 44000). The precursor molecular weight was chosen to be ten or more times the entanglement molecular weight. The precursor's relaxation follows the BSW spectrum $[61,62]$. Near the gel point, the plateau modulus of the entanglement region (intermediate time scales) is surprisingly little affected by the crosslinking (Fig. 12). Only at higher extents of crosslinking, beyond the gel point, does the plateau modulus start to increase significantly. A scaling relation between $S$ and $M$ was found to be valid for these materials:

$$
S \propto M^{3.4 n}
$$

where 3.4 is the well known scaling exponent of the viscosity-molecular weight relation.

For describing the observed molecular weight effects in chemical gels, we propose to distinguish three regions based on the precursor molecular 


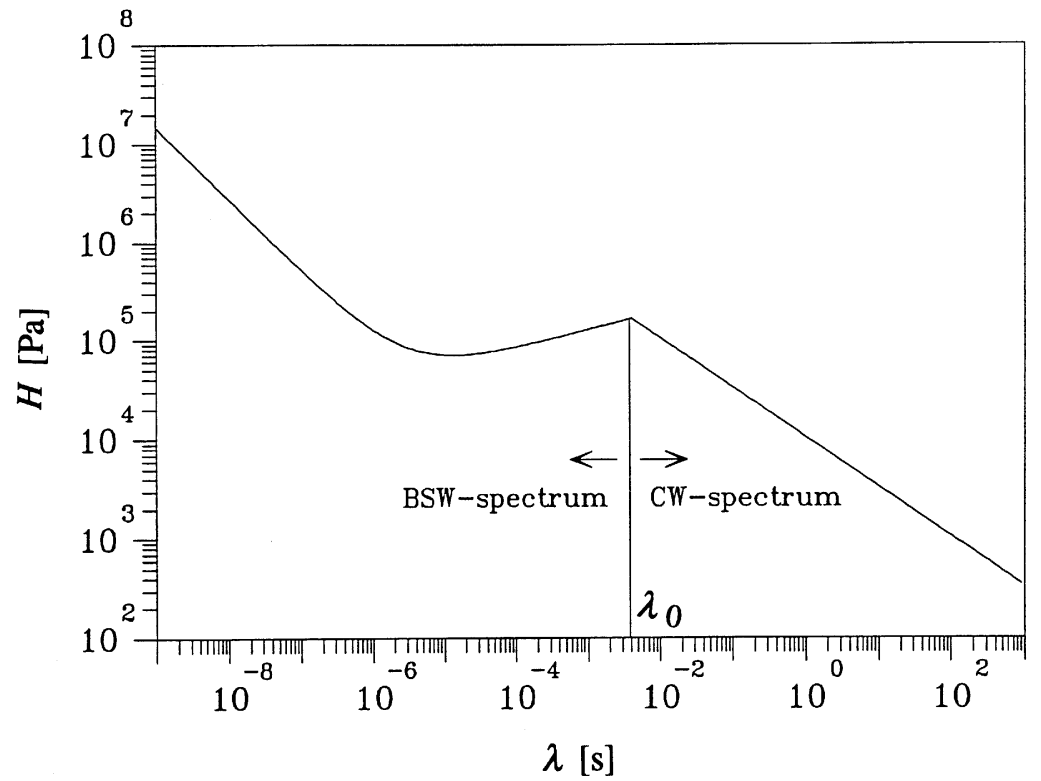

Fig. 11. Schematic of relaxation time spectrum of the critical gel of PBD $44\left(M_{w}=44000\right)$. The entanglement and glass transition is governed by the precursor's BSW-spectrum, while the CW spectrum describes the longer modes due to the crosslinking [60]. $\lambda_{0}$ denotes the longest relaxation time of PBD44 before crosslinking

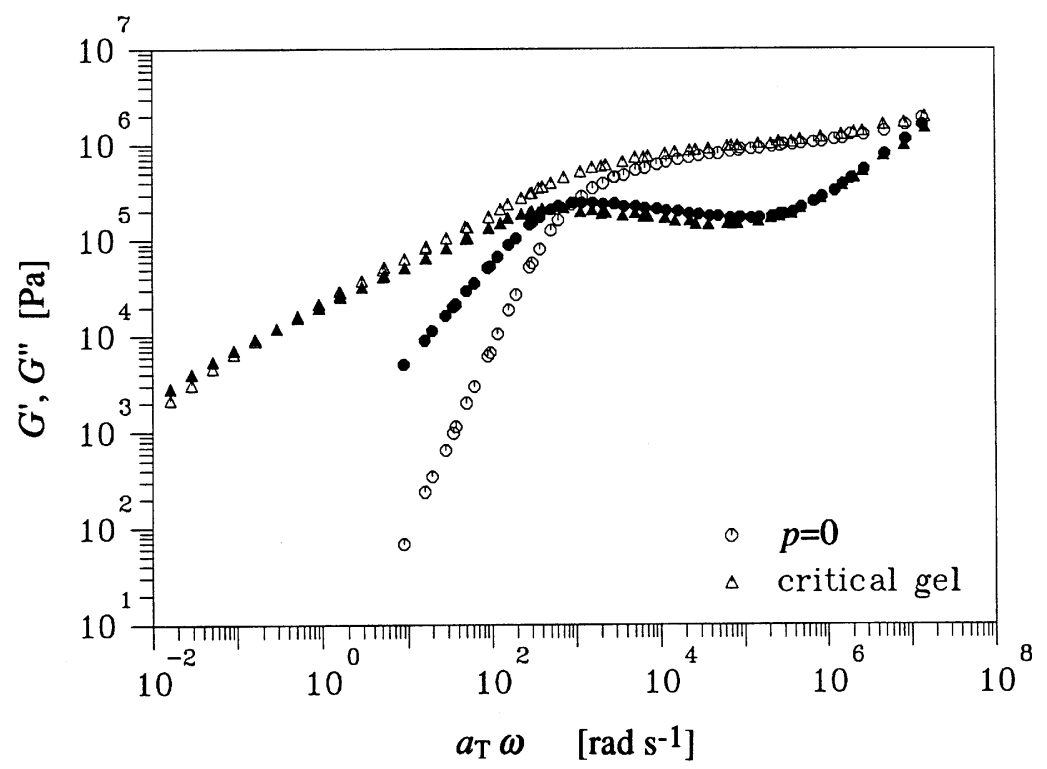

Fig. 12. Dynamic moduli master curves of PBD 44 precursor $(p=0)$ and PBD 44 critical gel [60]. The entanglement and glass transition regime is hardly affected by the crosslinking. Open symbols correspond to $G^{\prime}$, filled ones to $G^{\prime \prime}$ 
weight $M$ :

1. Low molecular weight $\mathrm{M}$ :

$$
M<M_{\text {GAUSS: }} \quad n \approx 0.7-0.8
$$

2. Intermediate molecular weight: $M_{\mathrm{GAUSS}}<M<M_{\mathrm{e}}: n \approx 0.4-0.7$

3. High molecular weight: $\quad M>M_{\mathrm{e}}$ : $\quad n \approx 0.4-0.5$.

$\mathrm{M}_{\text {GAUss }}$ is the molecular weight above which chains show Gaussian behavior. Very short chains, $M<M_{\mathrm{GAUSS}}$, associate into molecular clusters with nonGaussian building blocks, and the resulting critical gel is very stiff. Extensive data have been reported in the literature on these systems [38, 39, 63, 64]. Intermediate molecular weight precursors, $M_{\mathrm{GAUSS}}<M<M_{\mathrm{e}}$, already give much lower relaxation exponents $[5-7,18,58,65]$. The lowering of the relaxation exponent has been attributed to screening of excluded volume and hydrodynamic interaction [44].

Deficiency of cross-linker molecules (off-balancing of stoichoimetry) was found to increase the relaxation exponent value $[7,65,66]$. The gel becomes more 'lossy', and stress relaxation is accelerated. Adding of a non-reacting low molecular weight solvent also increases the relaxation exponent [58,65], even in physical gels [67]. Both effects have been attributed to screening [44, 65].

On the other hand, 'bulky' crosslinks as developed during the crystallization of polymer melts (no solvent) lower the relaxation exponent. The lowest values of $n$ which we have been able to generate so far occurred with physical gels in which the crosslinks consisted of large crystalline regions $[68,69]$.

This regular pattern in the relaxation exponent has been recognized for a wide range of chemically and physically gelling systems. The full range of gel properties should be explored further and should be utilized technically. The molecular or structural origin of these variations is not yet known to the extent where quantitative predictions could be derived from first principles. From a practical point of view, it is advantageous that the relaxation exponent is non-universal, since it allows us to prepare nearly critical gels with a wide range of properties as needed for specific applications.

\subsection{Viscoelastic Material Functions Near LST}

The simplest expression incorporating the basic features of self-similarity and cut-off for nearly critical gels has the spectrum of the critical gel altered by a cut-off at the longest time [19]:

$$
H(\lambda, p)=\left\{\begin{array}{ll}
\frac{S}{\Gamma(n)} \lambda^{-n} & \text { for } \lambda_{0}<\lambda<\lambda_{\max }(p) \\
0 & \text { for } \lambda>\lambda_{\max }(p)
\end{array},\right.
$$

The same form of self-similar spectrum will be applied to the sol and the transient part of the gel. The consequences of this most simple spectrum will be 
explored in the following. Introducing Eq. 3-6 into the equation for the relaxation modulus, Eq. 1-4, gives

$$
G(t)=G_{\mathrm{e}}(p)+\frac{S}{\Gamma(n)} \int_{0}^{\lambda_{\max }(p)} \lambda^{-n} \mathrm{e}^{-t / \lambda} \frac{d \lambda}{\lambda}
$$

The diverging longest relaxation time, Eq. 1-6, sets the upper limit of the integral. The solid (gel) contribution is represented by $G_{\mathrm{e}}$. The crossover to any specific short-time behavior for $\lambda<\lambda_{0}$ is neglected here, since we are mostly concerned with the long-time behavior.

We can also calculate other viscoelastic properties in the limit of low shear rate (linear viscoelastic limit) near the LST. The above simple spectrum can be integrated to obtain the zero shear viscosity $\eta_{0}$, the first normal stress coefficient $\psi_{1}$ at vanishing shear rate, and the equilibrium compliance $J_{\mathrm{e}}^{0}$ :

$$
\begin{aligned}
& \eta_{0}(p)=\int_{0}^{\lambda_{\max }} H(\lambda) d \lambda=\frac{S \lambda_{\max }^{1-n}}{(1-n) \Gamma(n)}, \\
& \psi_{1}(p)=2 \int_{0}^{\lambda_{\max }} H(\lambda) \lambda d \lambda=\frac{2 S \lambda_{\max }^{2-n}}{(2-n) \Gamma(n)}, \\
& J_{\mathrm{e}}^{0}=\frac{\psi_{1}}{2 \eta_{0}^{2}}=\frac{\Gamma(n)}{S} \frac{(n-1)^{2}}{2-n} \lambda_{\text {max }}^{n} .
\end{aligned}
$$

This most simple model for the relaxation time spectrum of materials near the liquid-solid transition is good for relating critical exponents (see Eq. 1-9), but it cannot be considered quantitatively correct. A detailed study of the evolution of the relaxation time spectrum from liquid to solid state is in progress [70]. Preliminary results on vulcanizing polybutadienes indicate that the relaxation spectrum near the gel point is more complex than the simple spectrum presented in Eq. 3-6. In particular, the relation exponent $n$ is not independent of the extent of reaction but decreases with increasing $p$.

\section{Constitutive Modeling with the Critical Gel Equation}

\subsection{The Critical Gel Equation}

Predictions using the observed relaxation time spectrum at the gel point are consistent with further experimental observations. Such predictions require a constitutive equation, which now is available. Insertion of the $\mathrm{CW}$ spectrum, Eq. 1-5, into the equation for the stress, Eq. 3-1, results in the linear viscoelastic constitutive equation of critical gels, called the 'critical gel equation'

$$
\tau(t)=n S \int_{-\infty}^{t}\left(t-t^{\prime}\right)^{-(n+1)} \mathbf{C}^{-1}\left(t ; t^{\prime}\right) d t^{\prime} \quad \text { at } p=p_{\mathrm{c}} .
$$


It may alternatively be expressed with the rate of strain tensor $\partial \mathbf{C}_{t}^{-1}\left(t ; t^{\prime}\right) / \partial t^{\prime}$

$$
\tau(t)=-S \int_{-\infty}^{t}\left(t-t^{\prime}\right)^{-n} \frac{\partial}{\partial t^{\prime}} \mathbf{C}^{-1}\left(t ; t^{\prime}\right) d t^{\prime} \quad \text { at } p=p_{\mathrm{c}} .
$$

The cross-over to the glass at short times (or to other short-time behavior) is neglected here, which is justified as long as we only try to predict the long-time behavior, which is most affected by the solidification process.

The critical gel equation is expected to predict material functions in any small-strain viscoelastic experiment. The definition of 'small' varies from material to material. Venkataraman and Winter [71] explored the strain limit for crosslinking polydimethylsiloxanes and found an upper shear strain of about 2, beyond which the gel started to rupture. For percolating suspensions and physical gels which form a stiff skeleton structure, this strain limit would be orders of magnitude smaller.

\subsection{Linear Viscoelastic Modeling of Critical Gels}

With the gel equation, we can conveniently compute the consequences of the self-similar spectrum and later compare to experimental observations. The material behaves somehow in between a liquid and a solid. It does not qualify as solid since it cannot sustain a constant stress in the absence of motion. However, it is not acceptable as a liquid either, since it cannot reach a constant stress in shear flow at constant rate. We will examine the properties of the gel equation by modeling two selected shear flow examples. In shear flow, the Finger strain tensor reduces to a simple matrix with a shear component

$$
\left(\mathbf{C}^{-1}\right)_{12}=\gamma\left(t ; t^{\prime}\right)
$$

and a difference on the diagonal

$$
\left(\mathbf{C}^{-1}\right)_{11}-\left(\mathbf{C}^{-1}\right)_{22}=\left(\gamma\left(t ; t^{\prime}\right)\right)^{2}
$$

where

$$
\gamma\left(t ; t^{\prime}\right)=\int_{t^{\prime}}^{t} \dot{\gamma}\left(t^{\prime \prime}\right) d t^{\prime \prime}
$$

is the shear strain between times $t^{\prime}$ and $t$. These components are inserted into Eq. 4-1 for calculating the shear stress and the first normal stress difference:

(a) Startup of shear flow at constant rate. An experiment is considered in which the material is initially kept at rest, $\dot{\gamma}=0$, so that it can equilibrate completely. Starting at time $t=0$, a constant shear rate $\dot{\gamma}_{0}$ is imposed. The resulting shear stress and normal stresses depend on the time of shearing. The shear stress response $\tau_{21}(t)$ of the critical gel is predicted as

$$
\tau_{21}(t)=\dot{\gamma}_{0} S \int_{0}^{t}\left(t-t^{\prime}\right)^{-n} d t^{\prime}=\frac{1}{1-n} \dot{\gamma}_{0} S t^{1-n} .
$$


The transient viscosity $\eta=\tau_{21}(t) / \dot{\gamma}_{0}$ diverges gradually without ever reaching steady shear flow conditions. This clarifies the type of singularity which the viscosity exhibits at the LST: The steady shear viscosity is undefined at LST, since the infinitely long relaxation time of the critical gel would require an infinitely long start-up time.

The corresponding first normal stress difference $N_{1}(t)=\tau_{11}(t)-\tau_{22}(t)$ as predicted from Eq. 4-2

$$
N_{1}(t)=n S \int_{-\infty}^{t}\left(t-t^{\prime}\right)^{-(n+1)} \gamma\left(t ; t^{\prime}\right)^{2} d t^{\prime}=\frac{2}{2-n} S \dot{\gamma}_{0}^{2} t^{2-n}
$$

also grows with time without ever reaching a steady value. The ratio of first normal stress coefficient and viscosity

$$
\frac{N_{1}}{\dot{\gamma}_{0}^{2}} \frac{\dot{\gamma}_{0}}{\tau_{21}}=\frac{2(1-n)}{2-n} t
$$

grows linearly with time. The relaxation exponent $n$ solely determines the slope while the front factor cancels out. In experimental studies, the linear growth can be used as a convenient reference for finding the limits of linear response in this transient shear experiment (Fig. 13).

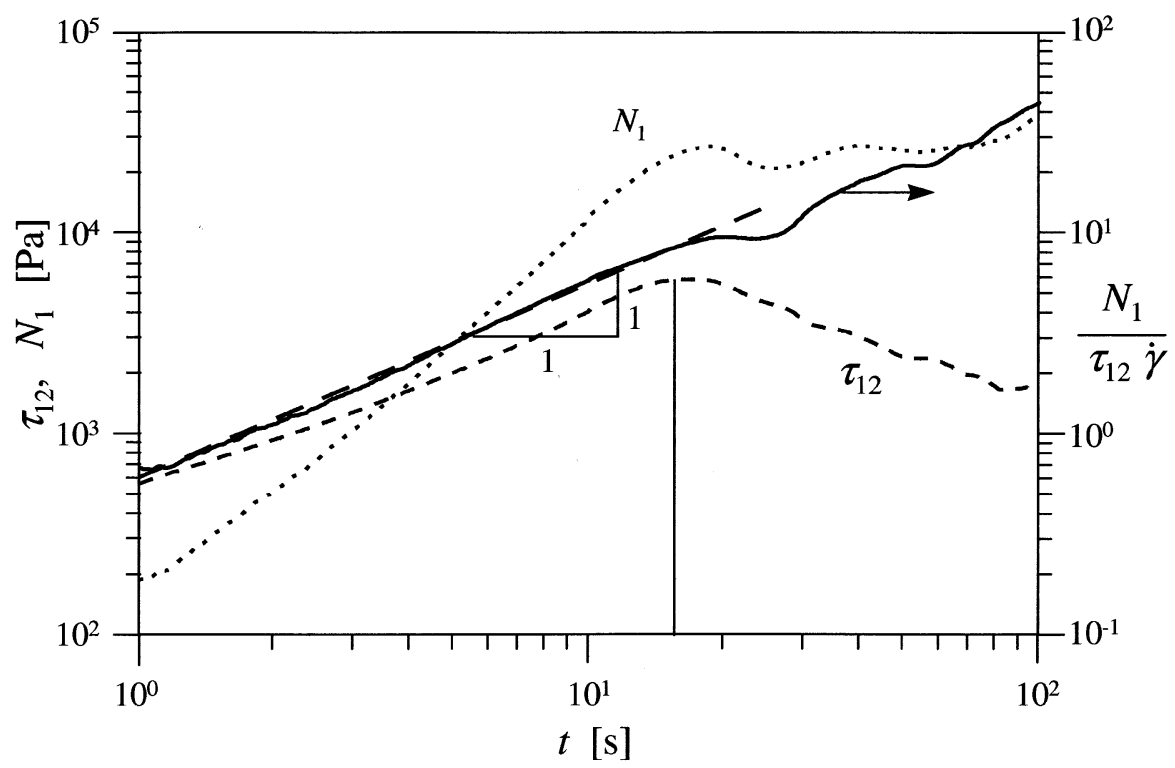

Fig. 13. Shear stress $\tau_{12}$ and first normal stress difference $N_{1}$ during start-up of shear flow at constant rate, $\dot{\gamma}_{0}=0.5 \mathrm{~s}^{-1}$, for PDMS near the gel point [71]. The broken line with a slope of one is predicted by the gel equation for finite strain. The critical strain for network rupture is reached at the point at which the shear stress attains its maximum value 
(b) Oscillatory shear. In a frequently used experiment, the sample is subjected to oscillatory shear at small amplitude $\gamma_{0}$. Prescribing a sinusoidal shear strain $\gamma(t)$ with an angular frequency, $\omega=2 \pi f[\mathrm{rad} / \mathrm{s}]$, which is defined by the number of cycles per time given by the frequency $f[\mathrm{~Hz}]$,

$$
\gamma(t)=\gamma_{0} \sin (\omega t)
$$

in Eq. 4-2 and determining the resulting shear stress

$$
\tau_{21}(t)=G^{\prime}(\omega) \gamma_{0} \sin (\omega t)+G^{\prime \prime}(\omega) \gamma_{0} \cos (\omega t)
$$

results in the following functional form of the dynamic moduli [10], the storage modulus $G^{\prime}$ and the loss modulus $G^{\prime \prime}$, at the gel point

$$
\begin{gathered}
G_{\mathrm{c}}^{\prime}(\omega)=\frac{G_{\mathrm{c}}^{\prime \prime}(\omega)}{\tan (n \pi / 2)}=S \Gamma(1-n) \cos (n \pi / 2) \omega^{n} \\
\text { for } 0<\omega<1 / \lambda_{0}, p=p_{\mathrm{c}} .
\end{gathered}
$$

Since $G^{\prime}$ and $G^{\prime \prime}$ are proportional to each other, the famous Cole-Cole plots [72] in which $\eta^{\prime \prime}(\omega)$ is plotted vs. $\eta^{\prime}(\omega)$ [or $G^{\prime}(\omega)$ is plotted vs. $\left.G^{\prime \prime}(\omega)\right]$ reduce to straight lines at the gel point.

The ratio of the two moduli is independent of frequency (Fig. 14)

$$
\tan \delta_{\mathrm{c}}=\frac{G_{\mathrm{c}}^{\prime \prime}}{G_{\mathrm{c}}^{\prime}}=\tan \frac{n \pi}{2} \text { for } 0<\omega<1 / \lambda_{0}, p=p_{\mathrm{c}} .
$$

which means that the 'flat' phase angle is a direct measure of the relaxation exponent [7]:

$$
n=\frac{2 \delta_{\mathrm{c}}}{\pi} \quad \text { for } 0<\omega<1 / \lambda_{0}, p=p_{\mathrm{c}} .
$$

Related material functions are the complex modulus

$$
G^{*}(\omega)=S \Gamma(1-n) \omega^{n}
$$

and the storage and loss compliance, $J^{\prime}(\omega)$ and $J^{\prime \prime}(\omega)$

$$
\begin{aligned}
& J^{\prime}(\omega)=\frac{G^{\prime}}{G^{2}+G^{\prime 2}}=\frac{\cos n \pi / 2}{S \Gamma(1-n)} \omega^{-n} \\
& J^{\prime \prime}(\omega)=\frac{G^{\prime \prime}}{G^{2}+G^{\prime 2}}=\frac{\sin \mathrm{n} \pi / 2}{S \Gamma(1-n)} \omega^{-n}
\end{aligned}
$$

(c) Creep and recovery behavior. Similar is the modeling procedure for the strain in a creep experiment. The most simple creep recovery experiment 


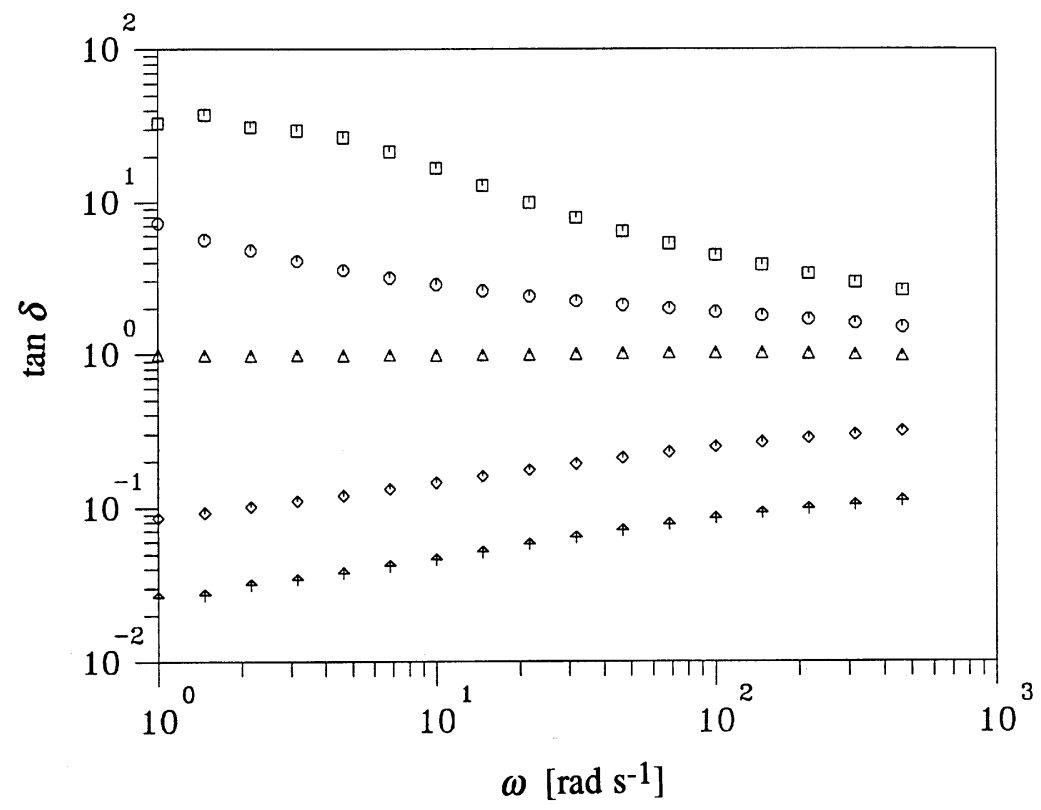

Fig. 14. Loss tangent of several stopped samples of vulcanizing polybutadiene $\left(M_{\mathrm{w}}=18000\right)$ [31]. At the gel point, $\tan \delta$ is frequency independent (flat curve in the middle). The relaxation exponent $n$ can be easily evaluated from the data $(\tan \delta=1$ yields $n=0.5)$

prescribes a pulse function

$$
\tau_{21}= \begin{cases}0 & \text { for } t<0 \\ \tau_{0} & \text { for } 0<t<t_{1} \\ 0 & \text { for } t_{1}<t\end{cases}
$$

where $\tau_{0}$ is the applied shear stress and $t_{1}$ is the creep time. The strain response of any linear viscoelastic material

$$
\gamma(t)=\int_{-\infty}^{t} J\left(t-t^{\prime}\right) \dot{\tau}\left(t^{\prime}\right) d t^{\prime}
$$

depends on the materials creep compliance $J\left(t-t^{\prime}\right)$. The classical relation between the creep compliance and the relaxation modulus [10]

$$
t=\int_{0}^{t} G(t-s) J(s) d s
$$

defines the creep compliance of the critical gel

$$
J(t)=\frac{1}{S} \frac{\sin (n \pi)}{n \pi} t^{n} \text { for } \lambda_{0}<t .
$$


The shear strain response has an analytical solution

$$
\gamma(t)=\frac{\tau_{0}}{S} \frac{\sin (n \pi)}{n \pi}\left(t^{n}-\left(t-t_{1}\right)^{n} h\left(1-t / t_{1}\right)\right) \text { for } t>0 .
$$

$h(x)$ is the Heaviside step function. It can be seen that neither the creep strain nor the strain rate will ever reach a steady value in finite times. When removing the stress, a complete recovery $\left(\gamma_{\infty}=0\right)$ is predicted for infinite times. This again is an example for the intermediate behavior between that of a liquid and a solid (Fig. 15).

(d) Retardation Time Spectrum. The relaxation behavior of critical gels can be represented equally well by the retardation time spectrum $L(\lambda)[73]$. Both are related by

$$
L(\lambda) H(\lambda)=\left(\left(\frac{1}{H(\lambda)} \int_{0}^{\infty} \frac{H(u)}{\lambda / u-1} \frac{d u}{u}\right)^{2}+\pi^{2}\right)^{-1}
$$

We determine the long time end of the retardation spectrum by approximating Eq. 1-5 with

$$
H(\lambda)=\frac{S}{\Gamma(n)} \lambda^{-n} \text { for } 0<\lambda<\infty
$$

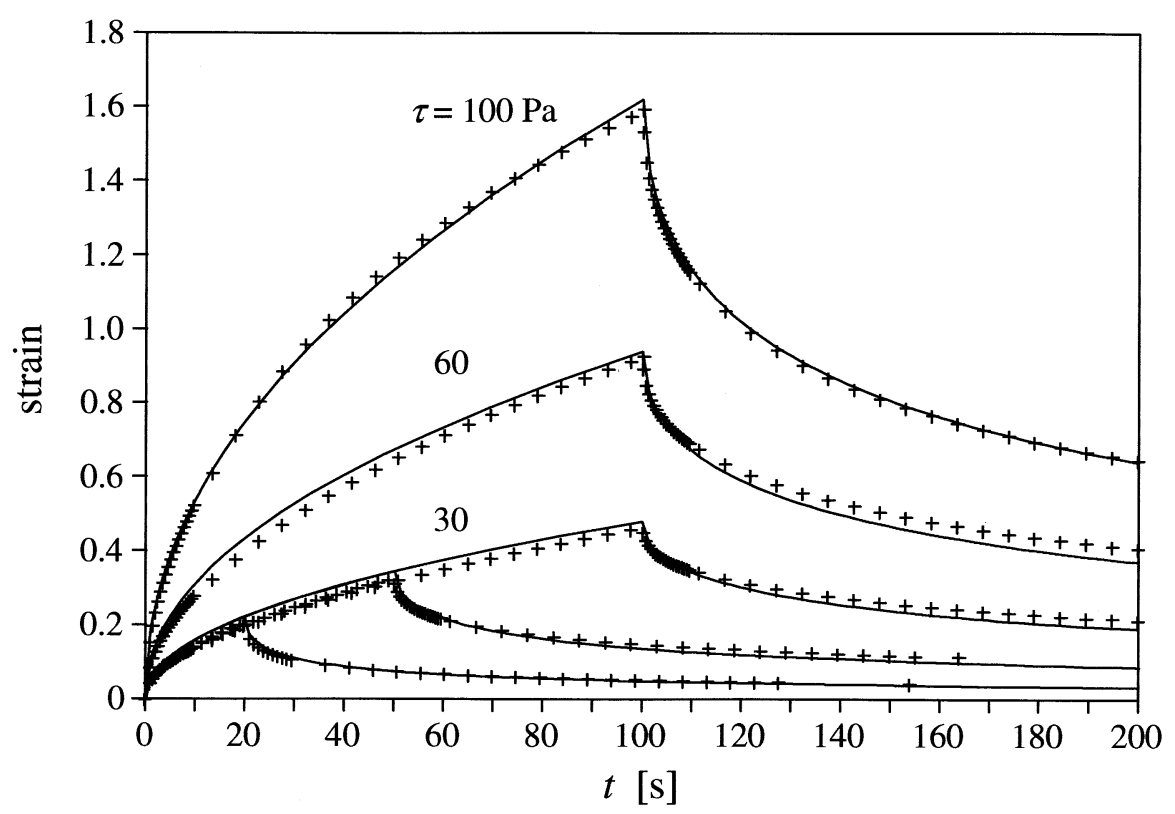

Fig. 15. Measured shear strain during creep under a constant shear stress and viscoelastic recovery after cessation of shear for PDMS near the gel point [71] plotted against the time. The solid lines are predicted by the gel equation for finite strain 
due to lack of better information in the time range $0<\lambda<\lambda_{0}$. Inserting Eq. 4-23 into Gross's relation, Eq. 4-22, leads to

$$
L(\lambda)=\frac{\Gamma(n)}{S} \frac{\sin ^{2}(n \pi)}{\pi^{2}} \lambda^{n} \text { for } \lambda_{0}<\lambda<\infty
$$

We assume that the above solution is valid in about the same time range as the self-similar relaxation time spectrum, Eq. 1-5. The retardation time spectrum is also self-similar. It is characterized by its positive exponent $n$ which takes on the same value as in the relaxation time spectrum.

\section{Physical Gelation}

The long range connectivity in the solidifying material may arise from physical phenomena instead of from chemical bonds. This process has been termed physical gelation. The large scale connectivity is meant in the sense that the motion of one molecule requires the motion of another molecule at considerable distance. This distance, called correlation length, increases with the advancement of the physical gelation process. The state at which the correlation length diverges defines the physical gel point. It is more difficult to define this gel point since in comparison with chemically crosslinking systems two of the most distinct criteria are missing: the molecular weight does not diverge and the system may be dissolved even after having passed the gel point.

At the beginning of the gelation process, more bonds are formed than are broken or dissolved. As a consequence, connectivity and correlation length grow in the material. However, this process cannot continue for long since the cluster size has a natural upper limit. This can be visualized by a simple argument. Let us consider a molecular cluster of $N$ physical bonds with characteristic lifetime, $\lambda_{\mathrm{b}}$. The average lifetime of this cluster is then $\lambda_{\mathrm{b}} / N$ and the survival probability is $\exp \left(-t N / \lambda_{\mathrm{b}}\right)$. As the cluster grows (increasing $N$ ), its survival probability decreases. The limit is reached at a maximum average cluster size, $N_{\max }$, at which the rate of bond breakage reaches the rate of bond formation. The characteristic time constant is then

$$
\lambda_{\mathrm{pg}}=\frac{\lambda_{\mathrm{b}}}{N_{\max }}
$$

where the subscript pg stands for physical gel. The material has a corresponding longest relaxation time, $\lambda_{\max }$. Early stages of cluster growth are governed by relaxation processes with a longest relaxation time which grows with the increasing connectivity. If $\lambda_{\max }$ exceeds $\lambda_{\mathrm{pg}}$, then the cluster does not survive the relaxation process; stress is released by breakage of clusters. This type of relaxation process has been studied by Cates $[74,75]$. 
We define a physical gel as a material which shows the gelation transition and has a $\lambda_{\max }$ after gelation which is orders of magnitude larger than before gelation. The characteristic equations at the gel point, Eqs. 1-1 and 1-5, need to be rewritten for a range of applicability $\lambda_{0}<\lambda<\lambda_{\text {pg }}$. The critical gel equation, Eq. 4-1, also needs to be rewritten to accommodate this upper limit. The most simple way to do this is by inserting Eq. 1-5 with modified upper limit $(\infty$ changed to $\lambda_{\mathrm{pg}}$ ) into Eq. 3-3:

$$
\tau(t)=n S \int_{-\infty}^{t}\left\{\frac{\Gamma\left(n+1,\left(t-t^{\prime}\right) / \lambda_{\mathrm{pg}}\right)}{\Gamma(n+1)}\right\}\left(t-t^{\prime}\right)^{-(n+1)} \mathbf{C}^{-1}\left(t ; t^{\prime}\right) d t^{\prime}
$$

where $\Gamma\left(n+1,\left(t-t^{\prime}\right) / \lambda_{\mathrm{pg}}\right)$ is an incomplete gamma function which is defined as

$$
\Gamma(n+1, x)=\int_{x}^{\infty} e^{z} z^{n} d z
$$

Strictly speaking, the physical gel at the gel point is still a liquid when observed at experimental times $t_{\mathrm{p}}$ which exceeds $\lambda_{\mathrm{pg}}$. We therefore define a new dimensionless group, the gel number $N_{\mathrm{g}}$

$$
N_{\mathrm{g}}=\frac{\lambda_{\mathrm{pg}}}{t_{\mathrm{p}}}=\frac{\text { lifetime of physical cluster }}{\text { process time }}
$$

The gelation transition is observable for $N_{\mathrm{g}}>10$. Otherwise, the material behaves as a liquid $\left(N_{\mathrm{g}}<1\right)$. Little is known about materials near $N_{\mathrm{g}}=1$. For the following, we consider only materials with $N_{\mathrm{g}} \gg 1$ and treat them just like chemical gels. The expression $\Gamma\left(n+1,\left(t-t^{\prime}\right) / \lambda_{\text {pg }}\right) / \Gamma(n+1)$ in Eq. $5-2$ approaches a value of one in this case of $N_{\mathrm{g}} \gg 1$, and the critical gel equation, Eq. 4-1, is recovered. However, much work is needed to understand the role of non-permanent physical clusters on network formation and rheological properties.

The closest relation to chemical gelation is found with physical network systems in which the network junctions originate from some physical mechanism such as crystallization, phase separation, ionic bonds, or specific geometric complexes. Such systems have been reviewed recently by the Nijenhuis [76] and Keller [77]. Physical networks have the potential advantage that the junctions open or close when altering the environment (temperature, pressure, $\mathrm{pH}$ ), i.e. the gelation process is reversible. The junctions, however, are less well-defined since their size and functionality (number of network strands which form a junction) varies throughout a sample. Their finite lifetime makes physical gels fluid-like in long-time applications, but it also allows them to heal if they get broken. The reversibility of junctions (and therefore connectivity) upon change of the environment in a physical gel is a characteristic feature which distinguishes it, for instance, from a highly entangled polymer melt or solution.

Beyond the notion of physical networks in which flexible strands are connected by junctions, we will use the term 'physical gelation' in the widest possible sense for polymeric systems which undergo liquid-solid transition due to any 
type of physical mechanism which is able to connect the polymer into large scale structures. These are quite manifold:

(a) Polymers lose their chain flexibility near their glass transition temperature and molecular motion correlates over longer and longer distances.

(b) Liquid crystalline polymers at their transition from nematic to smectic state gradually lose their molecular mobility.

(c) Suspensions in which the filler particles aggregate into sample spanning complexes.

The liquid-solid transition for these systems seems to have the same features as for chemical gelation, namely divergence of the longest relaxation time and power law spectrum with negative exponent.

Physical gelation responds strongly to stress or strain. The rate of bond formation, and therefore the growth rate of the correlation length, increases for some systems (increased rate of crystallization in semi-crystalline polymers; stress induced phase separation in block copolymers), but it also might decrease if the survival time of physical bonds is reduced by stress. Beyond the gel point, i.e. if the material is able to form sample spanning clusters which are characterized by multiple connectivity, physical gels are prone to creep under stress since bonds dissociate at a material-characteristic rate. This allows local relaxation of stress and reformation of physical bonds at reduced stress. In this case, breakage of a bond does not necessarily result in a reduction of the size of a cluster. Also, if stress is applied, the average lifetime of a bond, $\lambda_{b}$, decreases because of the energy input. This results in a reduction of $\lambda_{\text {pg }}$, which causes a reduction of the associated longest relaxation time, $\lambda_{\max }$.

\subsection{Physical Network Systems}

Physical network systems, especially crystallizing polymers, represent the most widely investigated physically crosslinking macromolecular systems $[68,69$, 78-81]. These systems comprise polymer melts and solutions in which network junctions are formed by small crystalline regions during the liquid-solid transition after cooling below the relevant melting temperature. The kinetics of this crystallization process depend on the degree of supercooling, $\Delta T$, and rheological properties are also influenced by $\Delta T$. A typical temperature profile for a crystallization experiment and the resulting evolution of fraction of crystalline polymer and dynamic moduli with time are shown schematically in Fig. 16. Although most crystallizing polymers exhibit the self-similar relaxation behavior at an intermediate state (Fig. 17 shows $\tan \delta$ at different times after cooling below $T_{\mathrm{m}}$ for a crystallizing polypropylene [68]), as commonly found in chemically crosslinking systems at the gel point, some systems showed no such power law relaxation at certain degrees of supercooling. This was attributed to non-uniform crystallization, which does not result in self-similar relaxation [69]. Also, the presence of melt state phase separation and residual high melting crystals can mask this characteristic relaxation pattern [68]. One of the 

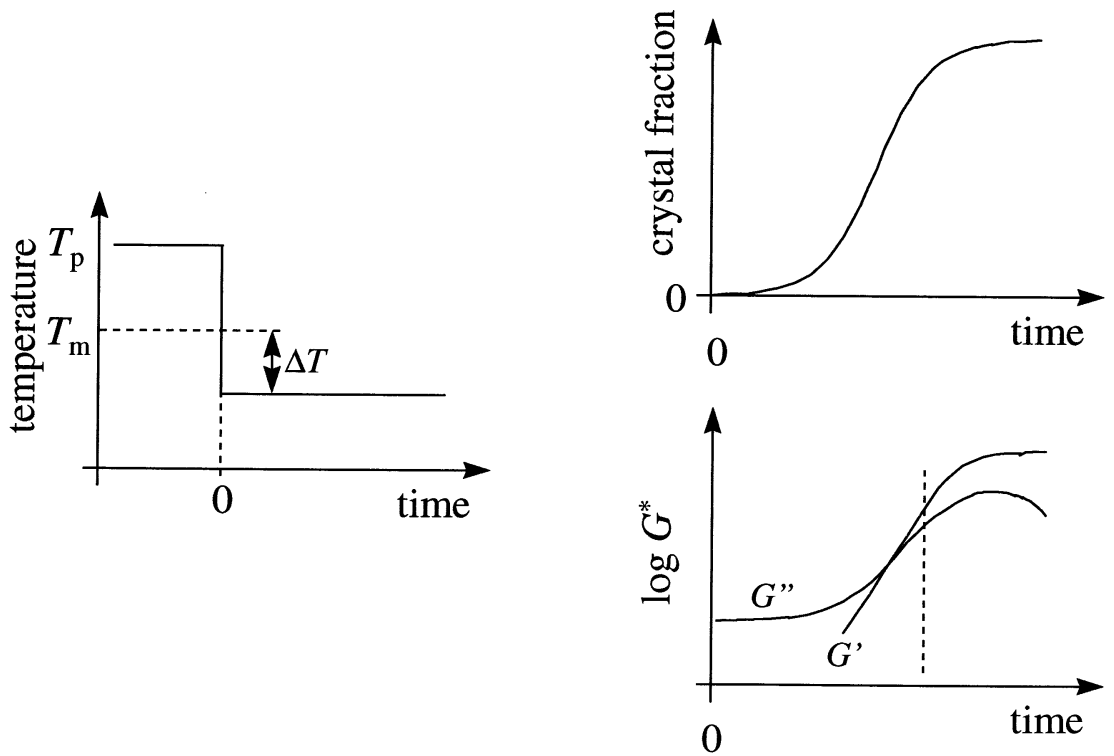

Fig. 16. Schematic of a typical temperature profile in a crystallization experiment and the resulting evolution of the fraction of crystalline polymer and dynamic moduli with time. The preheating temperature $T_{\mathrm{p}}$ is above the melting temperature $T_{\mathrm{m}}$

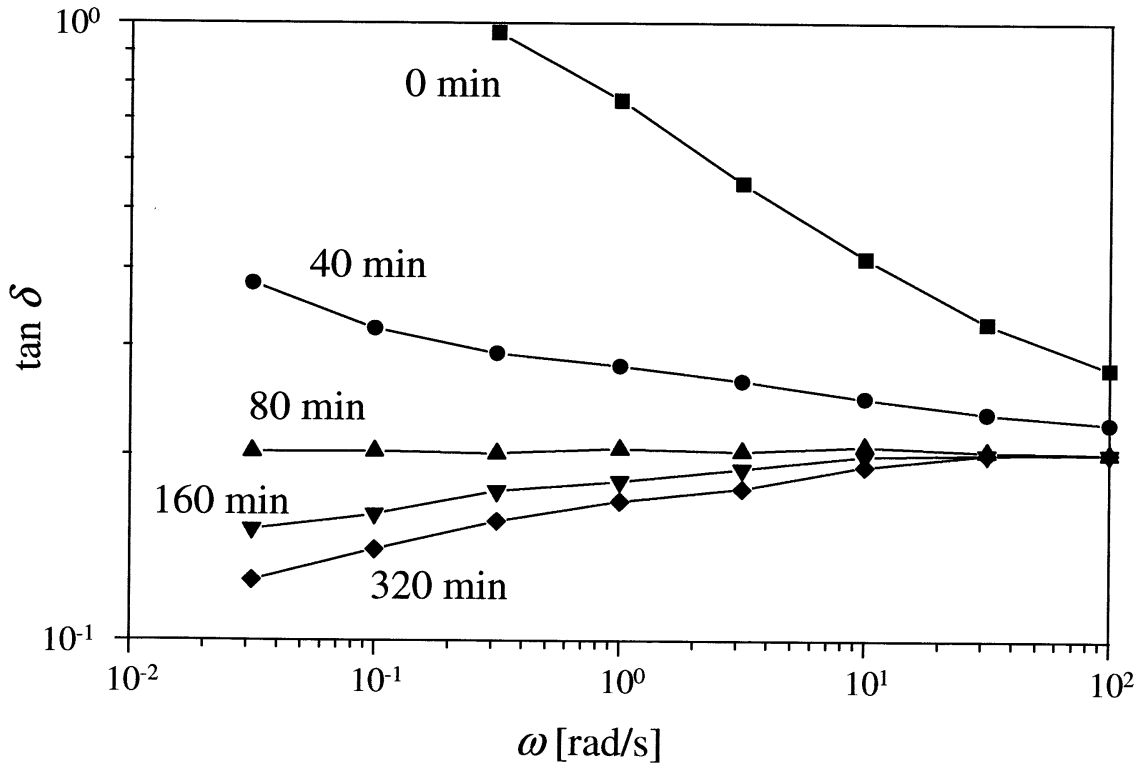

Fig. 17. Loss tangent of a crystallizing polypropylene at different times after cooling from $T=100^{\circ} \mathrm{C}$ to $T=40^{\circ} \mathrm{C}$ (below $T_{\text {melt }}$ ). Data from Lin et al. [68]. 
investigated systems, a bacterial polyester, followed the time-cure superposition principle in the liquid state up to the gel point [69]. This principle, however, could not be verified for samples beyond the gel point.

In general, low relaxation exponents $n$ (between 0.1 and 0.25 ) are characteristic for crystallizing polymers, although some systems are known which show a stronger dependence on frequency $(n \approx 0.7-0.8)$. A recent study on crystallizing isotactic polypropylene (iPP) resulted in a surprisingly low degree of crystallinity at the LST [80], which is a challenge to classical crystallization models. iPP forms a critical gel which is extremely soft (small $S$, large $n$ ).

Possible morphologies of partially crystalline polymers are shown in Fig. 18. Figure 18a depicts the case of small crystallites that act as physical crosslinks between polymeric chains, thus connecting those chains into a 3-dimensional network. In the case depicted in Fig. 18b, the material forms ribbon-shaped or needle-shaped crystalline regions in which different segments of a large number of chains are incorporated. This could explain the low degree of crystallinity at the LST as detected for the iPP system [80].

Reversible gelation is often encountered in bio-polymeric systems. Typical examples are solutions of polypeptide residues derived from animal collagen [82-84]. In these systems, ordered collagen-like triple helices form the physical crosslinks.

Microphase separated systems are also known to yield a physical network which results in the self-similar relaxation pattern at an intermediate state
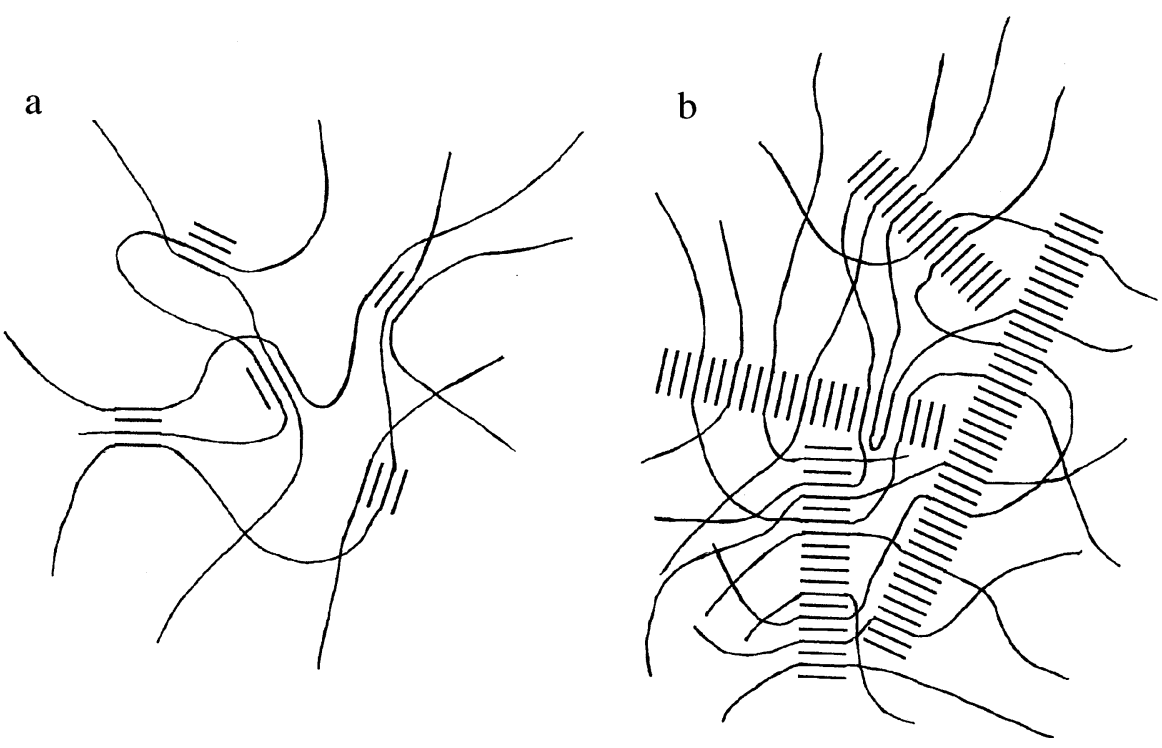

Fig. 18a, b. Possible morphologies of partially crystalline polymers. Small crystallites act as crosslinks (a); large ribbon-shaped or needle-shaped crystalline regions connect a large number of polymeric chains $(\mathbf{b})$ 
corresponding to the LST. Examples in which such behavior was observed are a segmented polyurethane elastomer with liquid crystalline hard segments (in which the phase-separated submicron mesophase acts as a provider of crosslinks below the isotropization temperature of the mesophase [85]) and several diblock and triblock copolymers with microphase separation below the ODT temperature $[86,87]$. A styrene-isoprene-styrene triblock copolymer system, for example, showed rapid microphase separation after cooling below the ODT temperature, while the large scale spatial order, resulting in a physical network, needed long annealing times. It was recently suggested that the disappearance of a terminal zone behavior, as encountered in block copolymers below the ODT temperature, is generally true for any polymer with layered structure [87].

Recently, it was shown that polydimethylcarbosiloxanes with a small content of side carbonyl groups (PDMS-C) exhibit increasing viscosity and formation of a physical network at elevated temperatures $[88,89]$. This was attributed to a rearrangement of intramolecular hydrogen bonds, which formed between the carboxyls during the synthesis and isolation of the polymers, forming intermolecular hydrogen bonds.

\subsection{Dynamic Glass Transition}

A glass transition is introduced dynamically when probing polymer molecules on such short time scales (in a high frequency dynamic experiment, for instance) that conformational rearrangements have no time to develop. The spectrum of polymers in this dynamic glass transition region is given by a power law with negative exponent. This has already been realized by Tobolsky [90], who introduced a wedge-box spectrum to describe entangled polymer melts where the 'wedge' (power law with negative slope) represented the relaxation behavior in the glass transition region. At long times this behavior is masked by the onset of the entanglement and/or flow regime. The glass transition spectrum looks like the CW spectrum for the critical gel except that it is cut off at a characteristic relaxation time, $\lambda_{\text {char }}$, i.e. the longest relaxation time is finite. This suggests that a polymer at the glass transition might be considered in the physical gel framework.

The amorphous solid state may be viewed as an extension of the liquid phase below a characteristic temperature. For low molecular weight materials, the atoms are frozen into their relative position at low temperatures [91]; this is called configurational freezing. For high molecular weight materials such as polymers, the molecular conformations freeze in and arrest molecular motion (conformational freezing); the temperature of conformational freezing is called glass transition temperature. $T_{\mathrm{g}}$. $T_{\mathrm{g}}$ is above the temperature of configurational freezing. The conformational freezing results in an increase of the correlation length for molecular motion. The divergence of the correlation length denotes the instant of solidification; thus the relaxation of the material is similar to that 
of a physical gel. The cut-off time $\lambda_{\text {char }}(T)$ of the relaxation spectrum depends on temperature and becomes very long when the temperature is lowered towards the glass transition temperature, $T_{\mathrm{g}}$, but it remains finite at $T_{\mathrm{g}}$. Dynamic mechanical experiments near $T_{\mathrm{g}}$ are needed for exploring the applicability of the gelation framework here. A very comprehensive study of dynamic mechanical behavior near $T_{\mathrm{g}}$ has been given by Zorn et al. [92].

The glass transition involves additional phenomena which strongly affect the rheology: (1) Short-time and long-time relaxation modes were found to shift with different temperature shift factors [93]. (2) The thermally introduced glass transition leads to a non-equilibrium state of the polymer [10]. Because of these, the gelation framework might be too simple to describe the transition behavior.

\subsection{Liquid Crystalline Polymers at their Nematic-Smectic Transition}

Viscoelastic response of liquid crystalline polymers (LCP) is very sensitive to smectic-nematic and smectic-isotropic phase transitions. Typical side chain LCPs with mesogenic groups pendant to flexible backbones show liquid-like relaxation behavior at low frequencies in the nematic state, i.e. the storage modulus, $G^{\prime}$, is proportional to frequency, $\omega$, and the loss modulus, $G^{\prime \prime}$, is proportional to $\omega^{2}$. At intermediate frequencies, a power law dependence best describes the dynamic moduli [94, 95]. Chemically crosslinking polymers below the gel point show the same behavior, which is followed by a transition to entanglement and/or glass transition regime at higher frequencies. LCPs in the smectic phase do not exhibit a low frequency drop-off to liquid-like behavior, at least not in the experimentally observable frequency regime. $G^{\prime}$ and $G^{\prime \prime}$ seem to level off at low frequency, suggesting a more solid-type relaxation behavior. No real power-law dependence is observed in the smectic mesophase; however, at intermediate frequencies indications of self-similar relaxation can be observed.

\subsection{Suspensions}

Transition from liquid behavior to solid behavior has been reported with fine particle suspensions with increased filler content in both Newtonian and nonNewtonian liquids. Industrially important classes are rubber-modified polymer melts (small rubber particles embedded in a polymer melt), e.g. ABS (acrylonitrile-butadiene-styrene) or HIPS (high-impact polystyrene) and fiberreinforced polymers. Another interesting suspension is present in plasticized polyvinylchloride (PVC) at low temperatures, when suspended PVC particles are formed in the melt [96]. The transition becomes evident in the following 
experimental observations:

- The limiting storage modulus (at low frequencies) and relaxation modulus (at long times) become finite at high concentration, while they are zero at low concentration [97-102].

- The zero-shear viscosity and the dynamic viscosity (at low frequencies) diverge at high concentration, while they are constant at low concentration [99, 100, 102-105].

Liquid-solid transitions in suspensions are especially complicated to study since they are accompanied by additional phenomena such as order-disorder transition of particulates [98,106, 107], anisotropy [108], particle-particle interactions [109], Brownian motion, and sedimentation-particle convection [109]. Furthermore, the size, size distribution, and shape of the filler particles strongly influence the rheological properties $[108,110]$. More comprehensive reviews on the rheology of suspensions and rubber modified polymer melts were presented by Metzner [111] and Masuda et al. [112], respectively.

Oscillatory shear experiments are the preferred method to study the rheological behavior due to particle interactions because they directly probe these interactions without the influence of the external flow field as encountered in steady shear experiments. However, phenomena that arise due to the external flow, such as shear thickening, can only be investigated in steady shear experiments. Additionally, the analysis is complicated by the different response of the material to shear and extensional flow. For example, very strong deviations from Trouton's ratio (extensional viscosity is three times the shear viscosity) were found for suspensions [113].

We expect the liquid/solid transition to express itself in the same general relaxation patterns as chemical gelation, with a self-similar relaxation time spectrum at the gel point. A starting point for this hypothesis is the work of Castellani and Lomellini $[102,114]$, who compared the rheology of rubbermodified thermoplastics with increasing rubber content to the behavior during physical gelation. The relaxation spectra of ABS with different PBD content presented by Masuda et al. [112] also suggest this approach, since they seem to contain a power law at long times.

\section{Rheometry Near the Gel Point}

Viscoelastic properties on intermediate time scales are most appropriate for studying gelation. The stretching of the spectrum in the approach of the gel point (from either side), and the self-similarity of the spectrum at the gel point can best be observed by forcing the material through the transition while simultaneously measuring its continuously evolving linear viscoelastic properties. Small strain tests are preferable, since they avoid rupturing the fragile network structure. No specific equipment is required beyond what one would 
use for characterizing viscoelasticity in liquids or solids. Most common are rotational rheometers with concentric disk fixtures, cone and plate fixtures, or Couette geometry. Samples have to be prepared in the rheometer fixtures since they are too fragile and too sticky to be transferred later.

\subsection{Oscillatory Shear}

Small amplitude oscillatory shear is the method of choice for materials with very broad distributions of relaxation modes, such as materials near LST, and for materials which undergo change during the measurement. The dynamic moduli in Eq. 4-10 are defined by [10]

$$
\begin{array}{ll}
G^{\prime}(\omega)=G_{\mathrm{e}}+\omega \int_{0}^{\infty}\left[G(t)-G_{\mathrm{e}}\right] \sin (\omega t) d t & =\text { storage modulus, } \\
G^{\prime \prime}(\omega)=\omega \int_{0}^{\infty} G(t) \cos (\omega t) d t & =\text { loss modulus. }
\end{array}
$$

The above equations are generally valid for any isotropic material, including critical gels, as long as the strain amplitude $\gamma_{0}$ is sufficiently small. The material is completely characterized by the relaxation function $G(t)$ and, in case of a solid, an additional equilibrium modulus $G_{\mathrm{e}}$.

The basic advantages of small amplitude oscillatory strain (shear or extension) come through its spectroscopic character, the experimental time for taking a single data point being roughly equal to the period of the strain wave, $2 \pi / \omega$. This allows to measure specific relaxation modes with time constants in the order of $1 / \omega$ independently of any longer or shorter modes which might be present in the polymer, i.e. only a small fraction of the spectrum is actually sampled. This is shown in Fig. 19, where a single power law spectrum with negative exponent $(n=0.7)$ was used to calculate the integral kernels of the following equations at $\omega=\lambda / \lambda_{0}$.

$$
\begin{aligned}
& G^{\prime}(\omega)=\int_{0}^{\lambda_{\max }} H(\lambda) \frac{(\omega \lambda)^{2}}{1+(\omega \lambda)^{2}} \frac{d \lambda}{\lambda} \\
& G^{\prime \prime}(\omega)=\int_{0}^{\lambda_{\max }} H(\lambda) \frac{\omega \lambda}{1+(\omega \lambda)^{2}} \frac{d \lambda}{\lambda}
\end{aligned}
$$

The divergence of the longest relaxation time does not perturb the measurement. In comparison, steady state properties (the steady shear viscosity, for instance) would probe an integral over all relaxation modes and, hence, fail near the gel point. 


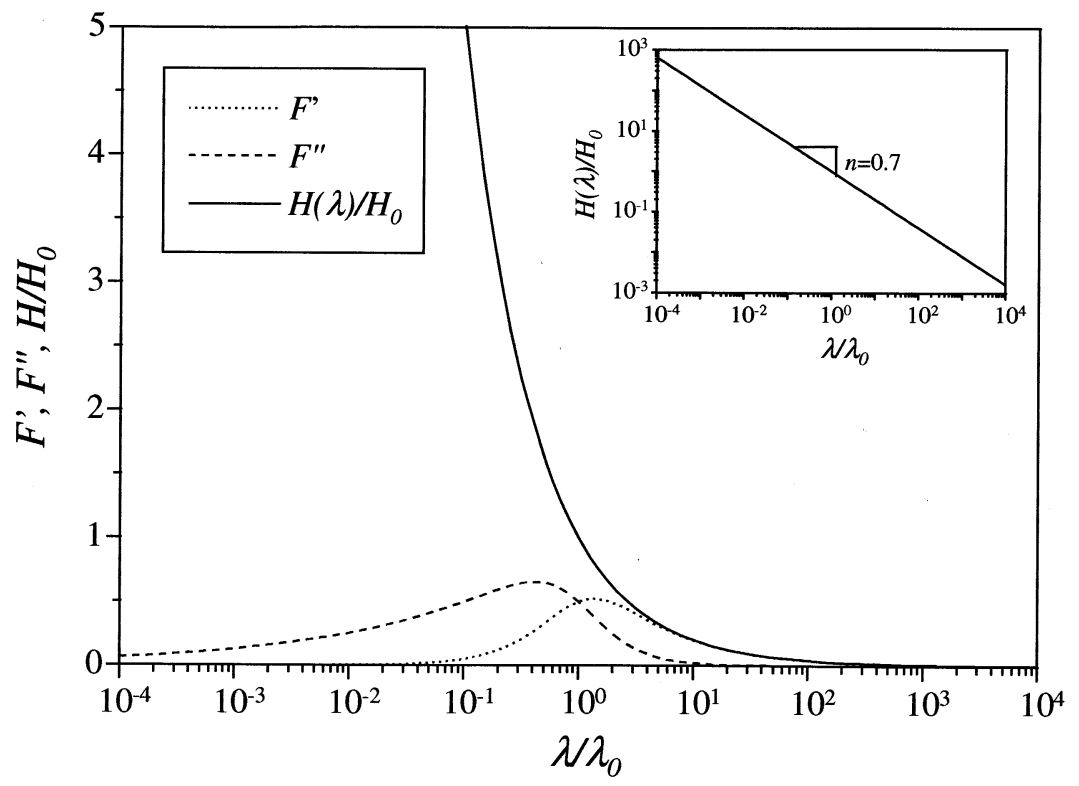

Fig. 19. $F^{\prime}\left(\lambda / \lambda_{0}\right)$ and $F^{\prime \prime}\left(\lambda / \lambda_{0}\right)$ for the evaluation of $G^{\prime}$ and $G^{\prime \prime}$ at $\omega=1 / \lambda_{0}$.

$$
\begin{aligned}
& G^{\prime}\left(\omega=1 / \lambda_{0}\right)=H_{0} \int_{0}^{\infty} F^{\prime}(x) d x \quad \text { with } F^{\prime}(x)=x^{-(n+1)} \frac{x^{2}}{1+x^{2}} \\
& G^{\prime \prime}\left(\omega=1 / \lambda_{0}\right)=H_{0} \int_{0}^{\infty} F^{\prime \prime}(x) d x \quad \text { with } F^{\prime \prime}(x)=x^{-(n+1)} \frac{x}{1+x^{2}}
\end{aligned}
$$

Insert: Reduced power law spectrum $H(\lambda)=H_{0}\left(\lambda / \lambda_{0}\right)^{-n}$

Typical for the spectroscopic character of the measurement is the rapid development of a quasi-steady state stress. In the actual experiment, the sample is at rest (equilibrated) until, at $t=0$, oscillatory shear flow is started. The shear stress response may be calculated with the general equation of linear viscoelasticity [10] (introducing Eqs. 4-3 and 4-9 into Eq. 3-2)

$$
\tau_{21}(t)=\int_{-\infty}^{0} 0+\int_{0}^{t} G\left(t-t^{\prime}\right) \omega \gamma_{0} \cos \left(\omega t^{\prime}\right) d t^{\prime}
$$

The first integral denotes the rest period, $-\infty<t^{\prime}<0$, where the strain rate is zero. The second integral contains a relaxation function which we chose very broad, including relaxation times much larger than the period $2 \pi / \omega$. Integration and quantitative analysis clearly showed (without presenting the detailed figures here) that the effect of the start-up from rest is already very small after one cycle 
and definitely is negligible after two cycles. Eq. 6-5 simplifies to

$$
\tau_{21}(t) \approx \int_{-\infty}^{t} G\left(t-t^{\prime}\right) \omega \gamma_{0} \cos \left(\omega t^{\prime}\right) d t^{\prime}
$$

The start-up time does not depend on the longest relaxation time of the material even if it is orders of magnitude larger than the period $2 \pi / \omega$ [115]. This is an important prerequisite for an experiment near LST.

The dynamic mechanical experiment has another advantage which was recognized a long time ago [10]: each of the moduli $G^{\prime}$ and $G^{\prime \prime}$ independently contains all the information about the relaxation time distribution. However, the information is weighted differently in the two moduli. This helps in detecting systematic errors in dynamic mechanical data (by means of the Kramers-Kronig relation [54]) and allows an easy conversion from the frequency to the time domain $[8,116]$.

Figures 20 and 21 show typical dynamic moduli and loss tangent distributions. These were measured by Chambon and Winter [5] on several partially crosslinked samples of PDMS.

Limitations of the experiment at low frequencies come from the long experimental times, during which the sample structure may change so much that the entire experiment becomes meaningless. At high frequencies, limitations

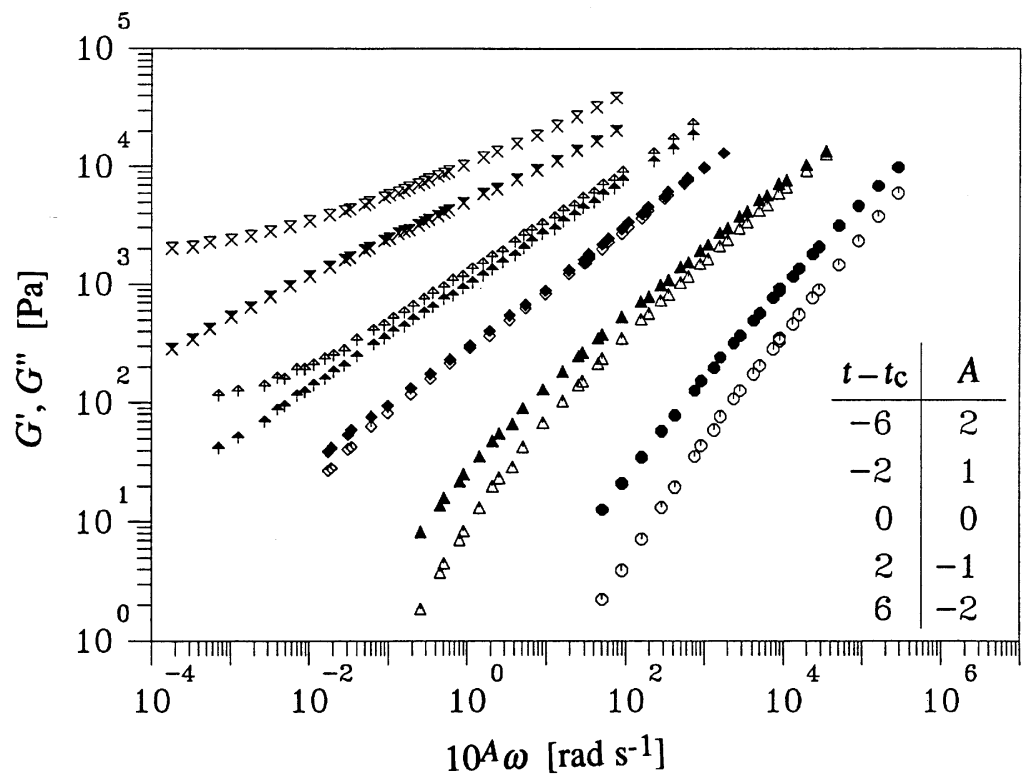

Fig. 20. Dynamic moduli, $G^{\prime}$ (open symbols) and $G^{\prime \prime}$ ( filled symbols), for five partially crosslinked PDMS samples at different extents of reactions (same data as Fig. 2). $G^{\prime}$ curves downward for the liquid and curves to the left for the solid. The straight lines belong to the sample which is very close to the gel point. $t-t_{\mathrm{c}}$ is given in minutes 


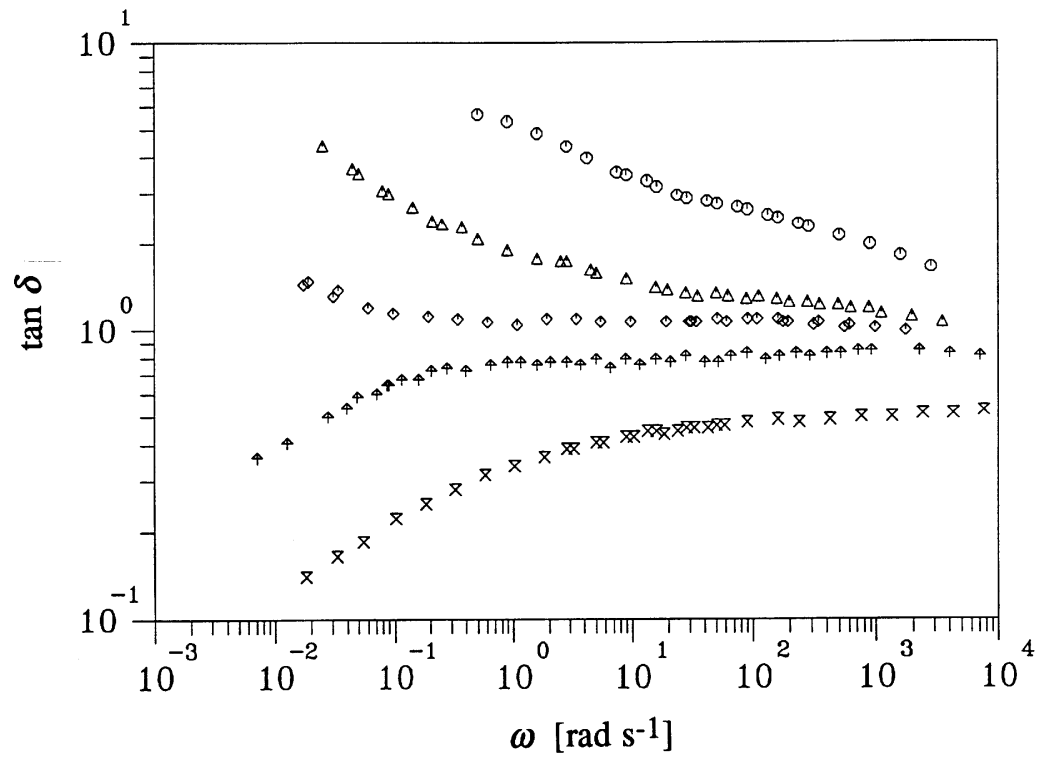

Fig. 21. Loss tangent, $\tan \delta$, for five partially crosslinked PDMS samples at different extents of reactions. For the liquid, a negative slope is observed. At the LST, $\tan \delta$ is independent of frequency. The loss tangent of the solid material exhibits a positive slope

arise from inertial effects. The effect of changes in the sample will be discussed next.

\subsection{Sample Mutation}

One really would like to know $G^{\prime}$ and $G^{\prime \prime}$ data over an as wide as possible frequency window of the sample at intermediate states during the transition. This has been achieved best for chemical gelation by stopping the reaction at intermediate extents $p$ with a catalyst poison [5] and then probing the stable samples (see Figs. 20 and 21). However, this stopping of the crosslinking is only possible for exceptional materials. More common is the situation where the transition process cannot be brought to a halt. The solidifying material has to be probed without stopping the reaction. Each data point in a sequence of measurements, having a sampling time $\Delta t$, belongs to a different state of the material. This is displayed schematically in Fig. 22. Since the properties at these intermediate frequencies evolve continuously, interpolation may be used to determine the properties at any time in between the measurements. The data are interpolated to obtain the material properties at discrete material states. By this procedure $[58,117,118]$, a range of properties is available at discrete states of the evolving material structure. 


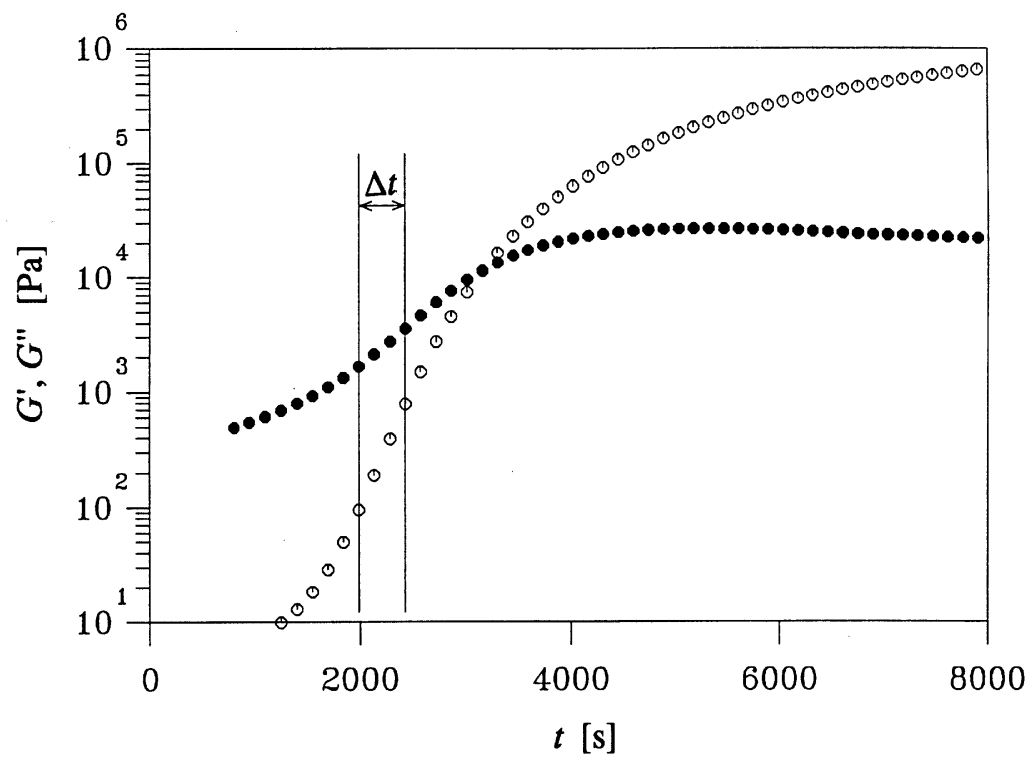

Fig. 22. Schematic evolution of dynamic moduli during crosslinking. The time period $\Delta t$ corresponds to the time necessary for a rheological measurement in case of a reacting sample. If the reaction is stopped, $\Delta t$ corresponds to the time in which the poison diffuses into the sample. The reaction is still carried on until the entire sample is poisoned. Then, the moduli remain constant

The effect of mutation is different in case of stopped samples, but the phenomenon cannot be completely avoided. Here, the experimental time period $\Delta t$ is determined by the poison diffusion. The catalyst poison solution is sprayed on top of a reacting sample and then diffuses into the core of the sample where it stops the reaction sequentially layer by layer. This leads to small inhomogeneity in the sample, since the reaction near the upper surface is stopped earlier than the reaction near the bottom of the mold.

Sample changes during the measurement might cause severe problems. The shear stress response of a crosslinking system exemplifies this nicely:

$$
\tau_{21}(t)=\int_{-\infty}^{t} G_{t^{\prime \prime}=t^{\prime}}^{t^{\prime \prime}=t}\left[t, t^{\prime}, p\left(t^{\prime \prime}\right)\right] \omega \gamma_{0} \cos \left(\omega t^{\prime}\right) d t^{\prime}
$$

The stress depends on the extent of reaction, $p\left(t^{\prime}\right)$, which progresses with time. However, it is not enough to enter the instantaneous value of $p\left(t^{\prime}\right)$. Needed is some integral over the crosslinking history. The solution of the mutation problem would require a constitutive model for the fading memory functional $G_{t^{\prime \prime}=t}^{t^{\prime \prime}=t}\left[t, t^{\prime}, p\left(t^{\prime \prime}\right)\right]$, which is not yet available. This restricts the applicability of dynamic mechanical experiments to slowly crosslinking systems. 
The mutation number $[117,119]$

$$
N_{\mathrm{mu}}=\Delta t \frac{1 \partial g}{g} \frac{\partial}{\partial t}
$$

gives an estimate of the relative changes of a measured variable $g$ (standing here for $G^{\prime}$ or $G^{\prime \prime}$ ) during the sampling time $\Delta t$. The spectroscopic character of dynamic mechanical experiments somehow alleviates the mutation problem. Mutation effects are considered negligible if the moduli $G^{\prime}$ and $G^{\prime \prime}$ change by $10 \%$ or less $\left(N_{\mathrm{mu}}<0.1\right)$ during the experimental time, $2 \pi / \omega$. Equivalent criteria apply to all the various material functions. The decisive dimensionless group (mutation number) has to stay below an acceptable tolerance level, $N_{\mathrm{mu}}<0.1$.

We mostly chose to probe each frequency individually to minimize the strain on the material and to expand the available frequency window. The experimental time can be reduced by simultaneously applying the sinusoidal strains of the lowest frequencies [120] and then quickly adding the higher frequency part of the spectral probing.

Time-resolved measurements on the changing sample have the advantages that the critical gel properties can be obtained from a single experiment and that a value for the rate of evolution of properties comes with the data.

\subsection{Time-Temperature Superposition}

Time-temperature superposition [10] increases the accessible frequency window of the linear viscoelastic experiments. It applies to stable material states where the extent of reaction is fixed ('stopped samples'). Winter and Chambon [6] and Izuka et al. [121] showed that the relaxation exponent $n$ is independent of temperature and that the front factor (gel stiffness) shifts with temperature

$$
S(T)=S\left(T_{0}\right) \frac{a_{T}{ }^{n}}{b_{T}} .
$$

This behavior is in between that of a liquid and a solid. As an example, PDMS properties obey an Arrhenius-type temperature dependence because PDMS is far above its glass transition temperature (about $-125^{\circ} \mathrm{C}$ ). The temperature shift factors are

$$
a_{\mathrm{T}}=\exp \left\{\frac{E}{R}\left(\frac{1}{T}-\frac{1}{T_{0}}\right)\right\} ; b_{T}=\frac{\rho\left(T_{0}\right) T_{0}}{\rho(T) T} .
$$

From the PDMS shift factors determined by Winter and Chambon, one may estimate that room temperature fluctuations affect the gel strength by no more than $5 \%$.

Time-temperature superposition at the gel point does not let us distinguish between the vertical and the horizontal shift, since the spectra are given by 
straight lines in a $\log / \log$ plot. We can only determine the total product $a_{T}^{n} / b_{T}$ [121] without being able to break it down into separate values $a_{T}$ and $b_{T}$. For polymers at temperatures far above the glass transition temperature $T_{g}$, however, the crosslinking does not seem to affect the shift factors in any major way and $a_{T}$ and $b_{T}$ values may approximately be taken from the temperature dependence of the precursor viscosity and the modulus of the fully crosslinked system, respectively. This has been found for crosslinking polycaprolactones far above the glass transition temperature [121]. More experiments are needed to confirm this very simple relation. It is expected to loose its validity for samples for which crosslinking strongly increases $T_{g}$.

\subsection{Time-Cure Superposition}

Measurement of the equilibrium properties near the LST is difficult because long relaxation times make it impossible to reach equilibrium flow conditions without disruption of the network structure. The fact that some of those properties diverge (e.g. zero-shear viscosity or equilibrium compliance) or equal zero (equilibrium modulus) complicates their determination even more. More promising are timecure superposition techniques [15] which determine the exponents from the entire relaxation spectrum and not only from the diverging longest mode.

Adolf and Martin [15] postulated, since the near critical gels are self-similar, that a change in the extent of cure results in a mere change in scale, but the functional form of the relaxation modulus remains the same. They accounted for this change in scale by redefinition of time and by a suitable redefinition of the equilibrium modulus. The data were rescaled as $G^{\prime} / G_{\mathrm{e}}(p)$ and $G^{\prime \prime} / G_{\mathrm{e}}(p)$ over $\omega \lambda_{\max }(p)$. The result is a set of master curves, one for the sol (Fig. 23a) and one for the gel (Fig. 23b).

Time-cure superposition is valid for materials which do not change their relaxation exponent during the transition. This might be satisfied for chemical gelation of small and intermediate size molecules. However, it does not apply to macromolecular systems as Mours and Winter [70] showed on vulcanizing polybutadienes.

\subsection{Growth Rate of Moduli}

The rate of change through the transition has not been studied widely. However, the growth of $G^{\prime}$ and $G^{\prime \prime}$ due to the increasing network connectivity seems to follow a regular pattern. For all our experiments of that type which were restricted by the accessible frequency range of the rheometer, the growth rate of $G^{\prime}$ at the gel point was typically twice as high as that of $G^{\prime \prime}$ :

$$
\left(\frac{1}{G^{\prime}} \frac{\partial G^{\prime}}{\partial p}\right)_{\omega} \cong 2\left(\frac{1}{G^{\prime \prime}} \frac{\partial G^{\prime \prime}}{\partial p}\right)_{\omega} \text { for finite } \omega .
$$



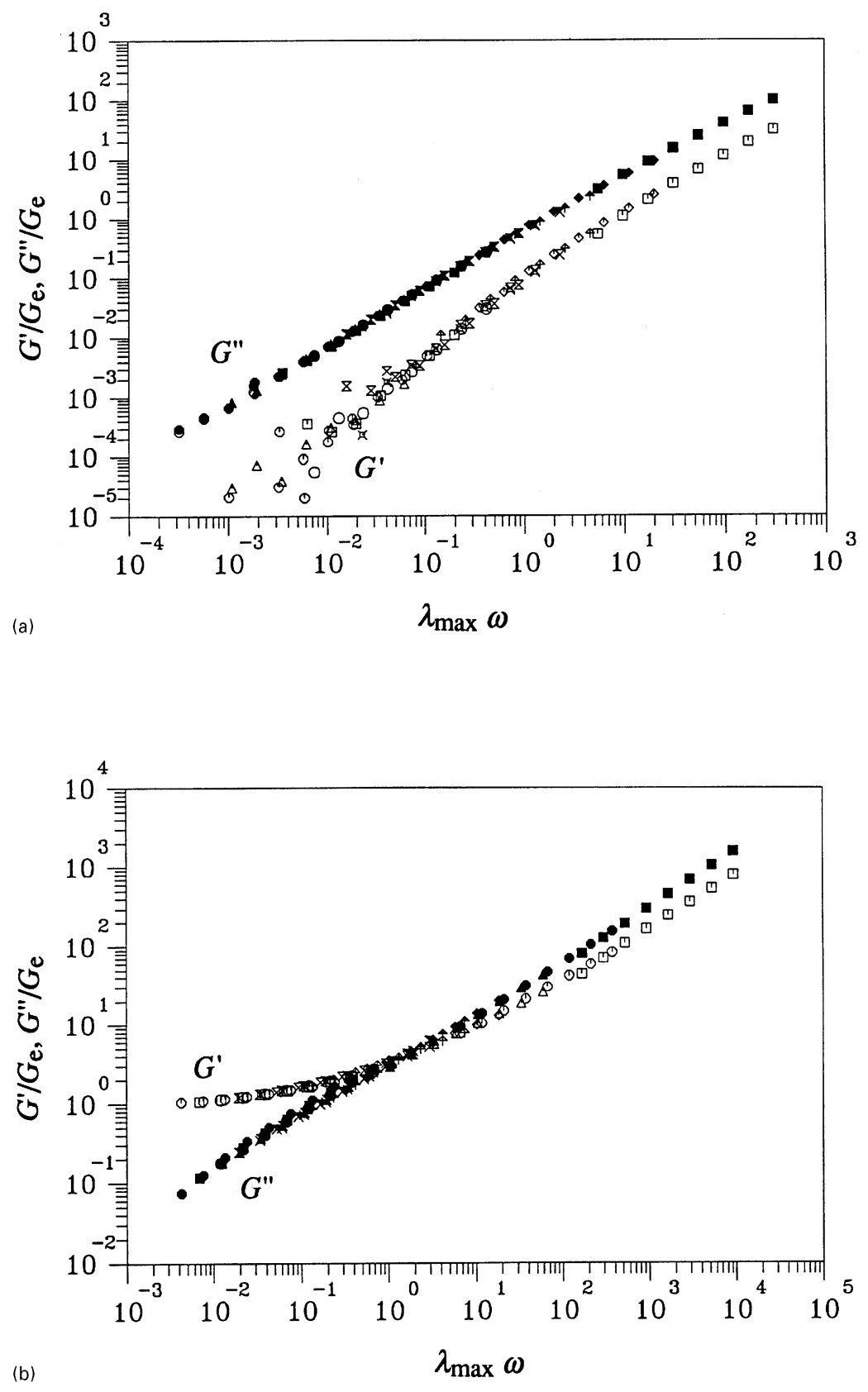

Fig. 23. Master curve obtained by time cure superposition of data on curing epoxy (a) before the LST and (b) after the LST [15] 


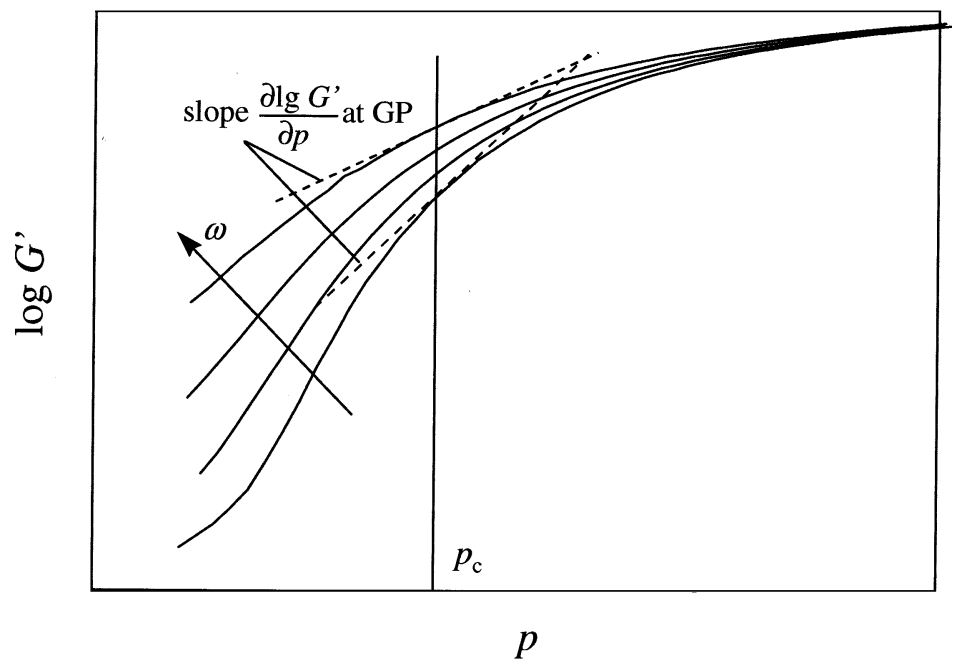

Fig. 24. Schematic of the evolution of the dynamic storage modulus, $G^{\prime}$, with extent of reaction, $p$, at four different frequencies

The growth rate decreases with frequency. Figure 24 shows this schematically for the storage modulus. The frequency dependence is the same for both $G^{\prime}$ and $G^{\prime \prime}$, and it also follows a power law (Fig. 25).

$$
\left(\frac{1}{G^{\prime}} \frac{\partial G^{\prime}}{\partial p}\right)_{\omega} \propto\left(\frac{1}{G^{\prime \prime}} \frac{\partial G^{\prime \prime}}{\partial p}\right)_{\omega} \propto \omega^{-\kappa} \text { for } p \text { near } p_{\mathrm{c}} .
$$

Precise knowledge of the critical point is not required to determine $\kappa$ by this method because the scaling relation holds over a finite range of $p$ at intermediate frequency. The exponent $\kappa$ has been evaluated for each of the experiments of Scanlan and Winter [122]. Within the limits of experimental error, the experiments indicate that $\kappa$ takes on a universal value. The average value from 30 experiments on the PDMS system with various stoichiometry, chain length, and concentration is $\kappa=0.214 \pm 0.017$. Exponent $\kappa$ has a value of about 0.2 for all the systems which we have studied so far. Colby et al. [38] reported a value of 0.24 for their polyester system. It seems to be insensitive to molecular detail. We expect the dynamic critical exponent $\kappa$ to be related to the other critical exponents. The frequency range of the above observations has to be explored further.

\subsection{Inhomogeneities}

The crosslinking process should not be considered to be completely homogeneous. Several phenomena might cause inhomogeneities. On a molecular scale, we 


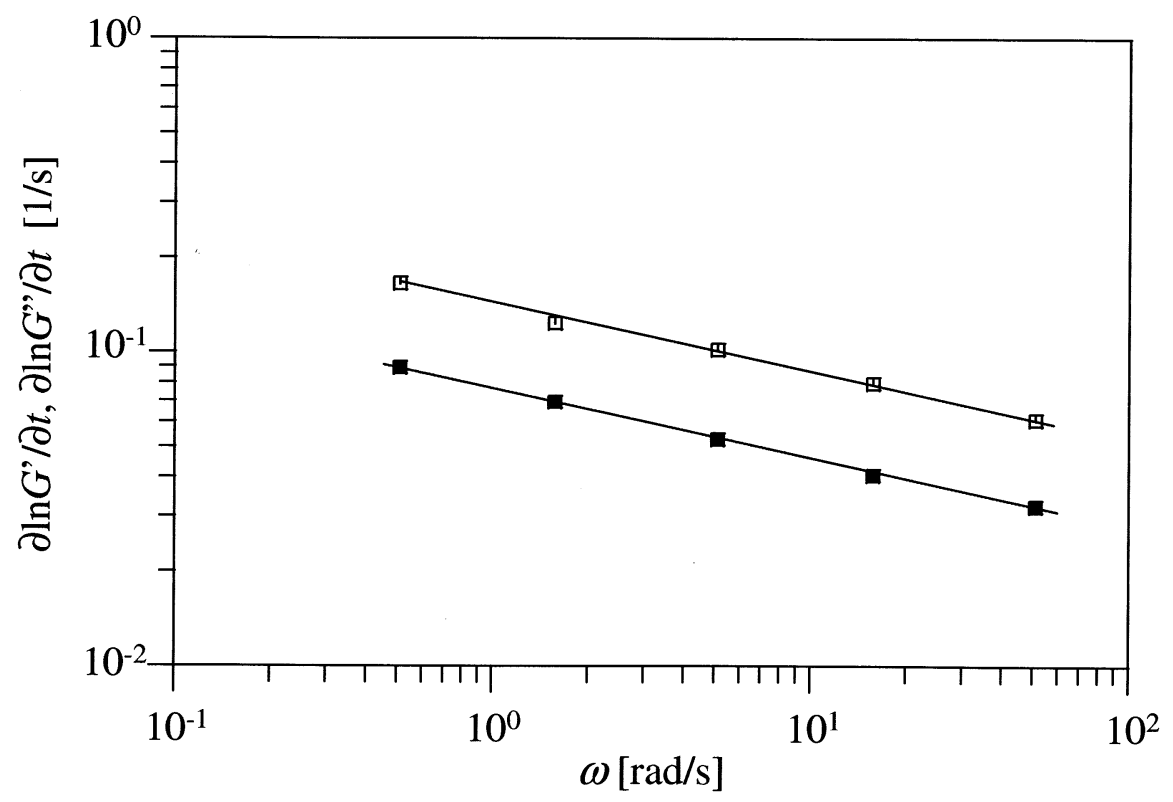

Fig. 25. Frequency dependence of moduli growth rate at the LST of crosslinking PDMS. Data from Scanlan and Winter [58]

can expect that fluctuations in crosslink density grow by excluding mobile sol molecules from regions of high crosslink density. This has been observed for highly crosslinked polymers and might occur at early stages of crosslinking.

Wall effects might also lead to inhomogeneities on the molecular level. Two possibilities can be envisioned: exclusion of large molecular clusters from the layer adjacent to a solid boundary, or adhesion of the largest clusters to the wall (while smaller molecules come and go near the wall).

These small-scale inhomogeneities would not be visible in rheological experiments since they average out in a macroscopic approach. However, there are inhomogeneities which do affect the macroscopic experiment. Most easily encountered is a temperature gradient which leads to gradients in the rate of reaction. The hotter region of the sample reaches the gel point first and the entire transition phenomenon gets smeared out. A clear gel point cannot be detected macroscopically. We have encountered this phenomenon quite often and use the purity of the self-similar behavior as an important criterion for a well-defined experiment.

Another typical example of inhomogeneity in rheometry is the oxidation of a polymer in a rotational rheometer in which a disk-shaped sample is held between metal fixtures. The oxygen enters the sample through the free surface (at the outer diameter) and diffuses radially inwards. The result is a radial gradient in properties which changes with time. If the reaction with oxygen results in 
a solidification, then the outer edge of the sample solidifies much before the inner regions, thus leading to an experiment-inherent inhomogeneity of the sample.

\section{Detection of the Liquid-Solid Transition}

The gel point is reached when the largest molecular or supermolecular cluster diverges to infinity [123]. This cannot be measured directly, but the consequences are so dramatic that they can be seen in a wide range of phenomena. Detection methods involve static light scattering on diverging clusters and dynamic light scattering with diverging correlation time [124-128], dissolving in a good solvent and extraction of a sol fraction [129], permeability [130], nuclear magnetic resonance spectroscopy [131], differential scanning calorimetry [132-134], and infrared [29, 135] and Raman spectroscopy $[136,137]$. Very sensitive indicators are rheological properties. Viscosity, first normal stress difference coefficient, equilibrium compliance, and longest relaxation time diverge at the gel point and the distribution of relaxation modes adopts a power law. These rheological features have been used extensively for detecting the gel point, and the following discussion will focus on rheological methods.

During our early experiments on chemical gels, when first observing the intermediate state with the self-similar spectrum, Eq. 1-5, we simply called it 'viscoelastic transition'. Then, numerous solvent extraction and swelling experiments on crosslinking samples showed that the 'viscoelastic transition' marks the transition from a completely soluble state to an insoluble state. The sol-gel transition and the 'viscoelastic transition' were found to be indistinguishable within the detection limit of our experiments. The most simple explanation for this observation was that both phenomena coincide, and that Eqs. 1-1 and 1-5 are indeed expressions of the LST. Modeling calculations of Winter and Chambon [6] also showed that Eq. 1-1 predicts an infinite viscosity (see Sect. 4) and a zero equilibrium modulus. This is consistent with what one would expect for a material at the gel point.

Physical solidification processes have no criterion for the gel point which would be as decisive as the molecular weight divergence of the sol-gel transition. LST measurements involve elaborate observations of flow to non-flow transition, and they rely on subjective judgement. The broadening of the spectrum into a power law distribution seems to coincide with such cessation of flow observations. We have therefore suggested that it should be valid to generalize from the behavior of chemical gels and identify LSTs of physical gels also by the CW spectrum [67]. In this way we can clearly identify both the approach to gelation and the transition of materials which otherwise would lack a clear definition. 


\subsection{Diverging Rheological Properties}

The diverging rheological properties are an unambiguous sign of the approaching gel point even if the measurement breaks down in its immediate vicinity (see Fig. 5). Macosko [138] gave a well-balanced overview of the methods up to 1985. The most common rheological tests for detecting LST measure the divergence of the steady shear viscosity [139-147] or the appearance of an equilibrium modulus [143, 146-148]. The equilibrium modulus evades accurate measurement near LST since its value is zero at LST and remains below the detection limit for a considerable time. It appears in a stress relaxation experiment as the long time limit of the relaxation function or in oscillatory strain as the low frequency asymptote of $G^{\prime}$.

Measurement of the diverging steady shear viscosity is an appealing experiment because of its simplicity. Even the torque on a processing machine might serve as an estimate of the diverging viscosity. It has, however, severe disadvantages that need to be considered [149]:

1. Near LST, the relaxation times become very long, and steady shear flow cannot be reached in the relatively short transient experiment. Large strains are the consequence for most reported data.

2. At large strain, the liquid shows shear thinning in some poorly understood fashion. Shearing causes breakage of the fragile network structure near LST, which has been observed as an apparent delay in gelation.

3. LST is found by extrapolation. The actual experiment may also end prematurely some time before LST if the developing structure in the material is very stiff and the rising stress overloads the rheometer.

The diverging viscosity, therefore, does not show the real gel point. The transition may appear early because of torque overload or it may be delayed by chain scission due to large strain. This apparent gel point, however, is still important since it relates to processing applications in which either the machine would clog or the newly formed network structure would break (or both).

\subsection{Monotonously Changing Properties}

The relaxation modulus evolves gradually during gelation. A set of data along the lines of Fig. 2 gives a good estimate of where the gel point occurs. The problem with it is that one cannot decide very well when exactly $G(t)$ has straightened out into a power law.

Dynamic mechanical properties also evolve gradually during the LST of polymeric systems. The gel point is reached when $\tan \delta$ becomes independent of frequency [58, 63, 65, 120, 149, 150] (see Eq. 4-12). Lines of $\tan \delta(t)$ at several frequencies $\omega_{1}, \omega_{2}, \omega_{3}, \omega_{4}, \omega_{5}$, etc. decay gradually and intersect at the gel point (see Fig. 26). The method is very effective. The instant of gelation can be measured as precisely as the accuracy of the rheometer permits - a significant 
advantage over extrapolation methods. An additional advantage is that the strain is kept small and shear modification of the molecular structure is avoided. The experiment not only tags the instant of gelation but also provides the value of the relaxation exponent $n$ (see Eq. 4-12).

Even before reaching the gel point, the converging lines can be extrapolated towards the expected gel point. The LST can be anticipated. This is convenient for preparing materials somewhere before the gel point but very close to it. The cross-linking can be stopped at a defined distance before LST, $\left(p_{\mathrm{c}}-p\right)$.

There are also some far-fetched proposals for the LST: a maximum in $\tan \delta$ [151] or a maximum in $G^{\prime \prime}$ [152] at LST. However, these expectations are not consistent with the observed behavior. The $G^{\prime \prime}$ maximum seems to occur much beyond the gel point. It also has been proposed that the gel point may be reached when the storage modulus equals the loss modulus, $G^{\prime}=G^{\prime \prime}[153,154]$, but this is contradicted by the observation that the $G^{\prime}-G^{\prime \prime}$ crossover depends on the specific choice of frequency [154]. Obviously, the gel point cannot depend on the probing frequency. Chambon and Winter $[5,6]$, however, showed that there is one exception: for the special group of materials with a relaxation exponent value $n=0.5$, the loss tangent becomes unity, $\tan \delta_{\mathrm{c}}=1$, and the $G^{\prime}-G^{\prime \prime}$ crossover coincides with the gel point. This shows that the crossover $G^{\prime}=G^{\prime \prime}$ does not in general coincide with the LST.

\subsection{Uniqueness of $\tan \delta$ Method}

A self-similar relaxation spectrum with a negative exponent $(-n)$ has the property that $\tan \delta$ is independent of frequency. This is convenient for detecting the instant of gelation. However, it is not evident that the claim can be reversed. There might be other functions which result in a constant $\tan \delta$. This will be analyzed in the following.

A constant loss tangent, $\tan \delta \neq f(\omega)$, requires dynamic moduli

$$
G^{\prime}=h(\omega), G^{\prime \prime}=A h(\omega) \rightarrow \tan \delta=G^{\prime \prime} / G^{\prime}=A=\text { const. }
$$

where $h(\omega)$ may be an arbitrary function. The question is whether $h(\omega)$ has to be a power law $h(\omega) \propto \omega^{n}$ or whether other functions are permitted. If other functions are permitted, then $\tan \delta \neq f(\omega)$ (for $1 / \lambda_{0}<\omega<\infty$ ) is not a unique criterion for the LST. For the following derivation, the short time behavior can be neglected since it has no effect on the ensuing argument. For simplicity we assume that the above condition applies over the entire frequency range, $0<\omega<\infty$. The Kramers-Kronig relation

$$
G^{\prime}(\omega)=\frac{2}{\pi} \int_{0}^{\infty} \frac{G^{\prime \prime}(x)}{1-(x / \omega)^{2}} \frac{d x}{x}
$$


can be evaluated for the above function

$$
h(\omega)=\frac{2}{\pi} \int_{0}^{\infty} \frac{A h(x)}{1-(x / \omega)^{2}} \frac{d x}{x}
$$

Substitution of $y=x / \omega$ gives

$$
1=A \frac{2}{\pi} \int_{0}^{\infty} \frac{h(y \omega)}{h(\omega)} \frac{1}{1-y^{2}} \frac{d y}{y}
$$

Any function of $y \omega$ with the factorization property

$$
h(y \omega)=k(y) h(\omega),
$$

where $k(y)$ may be an arbitrary function, is solution since it reduces the integral to some constant which defines $A$ :

$$
1=\frac{2 A}{\pi} \int_{0}^{\infty} \frac{k(y)}{1-y^{2}} \frac{d y}{y}
$$

A power law $h(\omega) \propto \omega^{n}$ satisfies this condition, Eq. 7-5, but any number of other functions with that property might be invented. The $\tan \delta$ criterion, therefore, might be not unique. However, no other material has yet been found which also obeys $\tan \delta=$ constant in the terminal frequency region, and we suggest the continued use of the $\tan \delta$ method for detecting LST until a counter example can be found.

\subsection{Determination of $S$ and $n$}

Dynamic mechanical data near the gel point allow easy determination of the parameters of the critical gel, Eq. 1-1. Tan $\delta$, as shown in Fig. 26, gives the relaxation exponent $n$

$$
n=\frac{2}{\pi} \tan ^{-1}\left(\frac{G^{\prime \prime}}{G^{\prime}}\right)
$$

$G^{\prime \prime} / G^{\prime}=\left(G^{\prime \prime} / G^{\prime}\right)_{\mathrm{c}}$ is the value where the curves intersect in a single point. The same data can be rearranged into

$$
S=\frac{G^{\prime}(\omega)}{\omega^{n} \cos (n \pi / 2) \Gamma(1-n)}
$$

$S$ has to be evaluated at the gel point (with $G^{\prime}=G_{\mathrm{c}}^{\prime}(\omega)$ at low frequencies). This completely characterizes the critical gel. The critical gel behavior is valid above a material characteristic time constant $\lambda_{0}$. The relation between $S$ and $n$ given by Eq. 3-4 holds only at the LST.

The above two equations are generally valid for viscoelastic liquids and solids. In this case, $n$ and $S$ would depend on frequency. In this sense, the above 


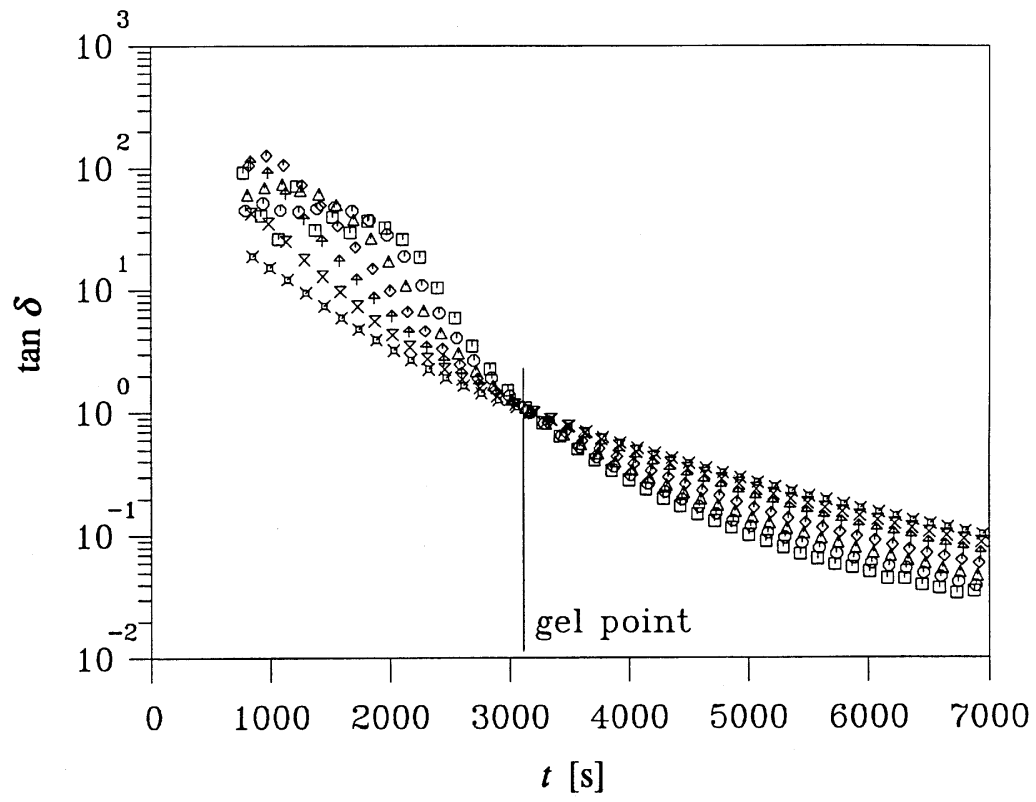

Fig. 26. Tan $\delta$ of a crosslinking $\operatorname{PBD}\left(M_{\mathrm{w}}=18000\right)$ as a function of reaction time [31]. Parameter is the frequency $\omega$. The polymer is vulcanized at the pendant vinyl units with a bifunctional silane crosslinker using a platinum compound as catalyst. The curves intersect at the gel point resulting in $\tan \delta \neq f(\omega)$

definitions are also valid in the vicinity of the gel point. However, $n$ and $S$ are only independent of frequency at the gel point where self-similar relaxation governs the rheological behavior.

\section{Other Observations of Power Law Relaxation}

Power law relaxation is no guarantee for a gel point. It should be noted that, besides materials near LST, there exist materials which show the very simple power law relaxation behavior over quite extended time windows. Such behavior has been termed 'self-similar' or 'scale invariant' since it is the same at any time scale of observation (within the given time window). Self-similar relaxation has been associated with self-similar structures on the molecular and supermolecular level and, for suspensions and emulsions, on particulate level. Such self-similar relaxation is only found over a finite range of relaxation times, i.e. between a lower and an upper cut-off, $\lambda_{1}$ and $\lambda_{\mathrm{u}}$. The exponent may adopt negative or positive values, however, with different consequences and 
limitations. A LST would require additional characteristics such as no upper time limit for the self-similar region, stretching out of the spectrum at the approach of the gel point and shrinking beyond the gel point, and different curvature of the storage modulus before and after the gel point. These characteristics have not been found in the following examples.

\subsection{Self-Similar Relaxation with Negative Exponent Value}

For negative exponent values, the symbol $-n$ with $n>0$ will be used. The self-similar spectrum has the form

$$
H(\lambda)=H_{0}\left(\frac{\lambda}{\lambda_{0}}\right)^{-n} \text { for } \lambda_{1}<\lambda<\lambda_{\mathrm{u}}
$$

The dimensionless relaxation exponent $n$ is allowed to take the values between 0 and 1 . The front factor $H_{0}$, with the dimension $\mathrm{Pa}$ and the characteristic time $\lambda_{0}$, depends on the specific choice of material. Various values have been assigned in the literature. The spectrum has only two independent parameters, since several constants are lumped into $\left(H_{0} \lambda_{0}^{-n}\right)$. For certain materials (the special case of LST), the upper limit of the power law spectrum may diverge to infinity, $\lambda_{\mathrm{u}} \rightarrow \infty$, without becoming inconsistent [18].

If the self-similar spectrum extends over a sufficiently wide time window, approximate solutions for the relaxation modulus $G(t)$ and the dynamic moduli $G^{\prime}(\omega), G^{\prime \prime}(\omega)$ might be explored by neglecting the end effects

$$
\begin{aligned}
& G(t)-G_{\mathrm{e}}=H_{0} \int_{0}^{\infty}\left(\frac{\lambda}{\lambda_{0}}\right)^{-n} \mathrm{e}^{-t / \lambda} \frac{d \lambda}{\lambda}=H_{0} \Gamma(n)\left(\frac{t}{\lambda_{0}}\right)^{-n} \\
& G^{\prime}(\omega)=H_{0} \Gamma(n) \Gamma(1-n) \cos \frac{n \pi}{2}\left(\lambda_{0} \omega\right)^{n} \\
& G^{\prime \prime}(\omega)=H_{0} \Gamma(n) \Gamma(1-n) \sin \frac{n \pi}{2}\left(\lambda_{0} \omega\right)^{n}
\end{aligned}
$$

These solutions of the idealized problem are a good approximation for the behavior within a time window $\lambda_{1}<t<\lambda_{\mathrm{u}}$ or the corresponding frequency window $1 / \lambda_{\mathrm{u}}<\omega<1 / \lambda_{1}$. Truncation effects can be seen near the edges $\lambda_{1}$ and $\lambda_{\mathrm{u}} \cdot \lambda_{0}$ is some material-specific reference time, which has to be specified in each choice of material, and $H_{0} \Gamma(n)=G_{0}$ is the corresponding modulus value.

The self-similar behavior is most obvious when it occurs in this form, i.e. if the exponent is negative and the self-similar region is extensive. $G(t), G^{\prime}(\omega)$, $G^{\prime \prime}(\omega)$, and $H(\lambda)$ all have power law format and they have been used interchangeably in the literature. Less obvious is the self-similar behavior for positive exponent values. 


\subsection{Self-Similar Relaxation with Positive Exponent Value}

For positive exponent values, the symbol $m$ with $m>0$ is used. The spectrum has the same format as in Eq. 8-1, $H(\lambda)=H_{0}\left(\lambda / \lambda_{0}\right)^{m}$, however, the positive exponent results in a completely different behavior. One important difference is that the upper limit of the spectrum, $\lambda_{\mathrm{u}}$, has to be finite in order to avoid divergence of the linear viscoelastic material functions. This prevents the use of approximate solutions of the above type, Eqs. 8-2 to 8-4.

Spectra with a positive exponent may be explored for the ideal case of power law relaxation over all times up to the longest relaxation time, $\lambda_{\max }$ :

$$
H(\lambda)=H_{0}\left(\frac{\lambda}{\lambda_{\max }}\right)^{m} \text { for } 0<\lambda<\lambda_{\max }
$$

$\lambda_{\text {max }}$ is always finite and is chosen here as the characteristic time of the spectrum. Even for this ideal spectrum, the relaxation modulus has to be evaluated numerically. It does not have any simple form which could be recognized as self-similar behavior. However, material functions can be evaluated for steady shear flow

$$
\begin{aligned}
& \eta_{0}=\int_{0}^{\lambda_{\max }} H(\lambda) d \lambda=\frac{H_{0} \lambda_{\text {max }}}{1+m} \\
& \psi_{1}=2 \int_{0}^{\lambda_{\max }} H(\lambda) \lambda d \lambda=\frac{2 H_{0} \lambda_{\max }^{2}}{2+m}
\end{aligned}
$$

\subsection{Observations of Self-Similar Relaxation Spectra}

A wide variety of polymeric materials exhibit self-similar relaxation behavior with positive or negative relaxation exponents. Positive exponents are only found with highly entangled chains if the chains are linear, flexible, and of uniform length [61]; the power law spectrum here describes the relaxation behavior in the entanglement and flow region.

Power law relaxation behavior is also expected (or has already been found) for other critical systems. Even molten polymers with linear chains of high molecular weight relax in a self-similar pattern if all chains are of uniform length [61].

Self-similar spectra with negative exponents are found in several different systems such as microgels [155], polymer blends and block copolymers [156] at their critical point, or coagulating systems at the threshold. Some broadly distributed polymers exhibit power law relaxation over an intermediate frequency range [157]. This behavior, however, is not related to gelation, which would require the power law to extend into the terminal zone, $\omega \rightarrow 0$. The relaxation spectrum of a polymer is broadened as long range connectivity develops (divergence of longest relaxation time), resulting in a power law 
behavior with negative exponent. Indications can also be seen in branched polymers [158] and in solutions containing polymeric fractals (e.g. flexible chain macromolecules of arbitrary self-similar connectivity [159] or natural objects with non-integer dimension such as aggregates or percolation clusters [160]).

Various types of power law relaxation have been observed experimentally or predicted from models of molecular motion. Each of them is defined in its specific time window and for specific molecular structure and composition. Examples are dynamically induced glass transition [90,161], phase separated block copolymers $[162,163]$, polymer melts with highly entangled linear molecules of uniform length $[61,62]$, and many others. A comprehensive review on power law relaxation has been recently given by Winter [164].

\section{Applications}

Processing of polymeric materials almost always involves a liquid-solid transition, and applications are often limited by solid-liquid transitions. Property changes are most dramatic near the transition. It is important to know where the transition occurs, how extensive the property changes are, and how fast they occur. Avoiding these transitions might often be the simplest solution, but that is not always an option. Some materials have to be produced, processed, or used near the gel point or up to the gel point. That is where rheological experiments permit the exact determination of the instant of gelation and the 'distance' from the gel point. We now can produce materials at controlled distances from the gel point and also process materials near the gel point. The critical gel properties serve as reference for expressing property changes in the vicinity of the gel point.

\subsection{Avoiding the Gel Point}

Mixing and shaping operations in polymer processing require sufficient molecular mobility which vanishes when the motion slows down near the gel point. Some materials only have small processing windows near the gel point (because of their limited chemical stability above their melting point, for instance, or because of their rapid crosslinking). Processing will become reasonably easy if such a narrow processing window can be targeted. This requires sufficiently accurate measurement of the material status relative to the location of the gel point. Instead of processing in the material characteristic window, one may consider shifting of the processing window by alteration of the material. This again requires detailed knowledge of the transition behavior and accurate methods for detecting the transition. 


\subsection{Materials Near the Gel Point}

Controlled sample preparation is difficult near the gel point where the rate of property change is largest. Physical gelation usually proceeds too rapidly so that the material near the gel point eludes the experiment or the application. However, chemical gelation is most suitable for controlling the evolving network structure. Several approaches have been explored in industrial applications and in research laboratories:

1. Polymerization chemistry was developed which allows the stopping of the crosslinking reaction in the vicinity of the gel point $[5,29]$.

2. Thermal quenching of the crosslinking reaction: In an actual reactive extrusion process, the degree of crosslinking can be controlled by adjusting the residence time at elevated temperature.

3. Off-balancing of stoichiometry by the right amount (depending on crosslinking system) allows preparation of materials near the gel point [66].

4. Crosslinking by controlled amounts of radiation. Examples are $\gamma$-irradiated polyethylenes [165] and UV-irradiated polyurethane [166]. Best contestants are endothermic reactions which require energy for the formation of each chemical bond and which cease crosslinking when the energy supply is turned off. The extent of reaction directly depends on the amount of absorbed energy. Radiation can be used to enter energy into transparent materials. The radiation intensity decreases along the path of the radiation in the materials. This potentially leads to samples with a large gradient in extent of reaction, the exposed side of the sample being further crosslinked than the backside. Not suitable for preparing polymers near the gel point are chemical reactions in which radiation only initiates the crosslinking reaction so that it continues even after the radiation has been turned off.

\subsection{Damping Materials}

Critical gels have a damping plateau instead of the commonly observed damping peak. The loss tangent, Eq. 4-12, is uniformly high over a wide frequency range, $0<\omega<1 / \lambda_{0}$. At higher frequencies, $\omega>1 / \lambda_{0}$, the usual glassy behavior sets in, or, if the critical gels are made from precursors of high molecular weight, the entanglement behavior dominates before glassy modes take over. The damping behavior is independent of temperature $[n \neq f(T)]$, which seems to be unique among polymeric materials.

The damping material does not have to be a critical gel. Many applications do not require extra low damping frequencies. The lowest vibration damping frequency $\omega_{\min }$ determines the longest relaxation time, $\lambda_{\max }$. A suitable damping material would be crosslinked beyond the gel point, with a $\lambda_{\max }$ of about $1 / \omega_{\min }$. 


\subsection{Pressure-Sensitive Adhesives}

Polymers at the gel point are extremely powerful adhesives. They combine the surface-wetting property of liquids with the cohensive strength of solids. The mechanical strength of an adhesive bond in composite materials (with crosslinking matrix) develops during the sol-gel transition, and the strength of pressuresensitive adhesives can be tailored through their degree of crosslinking. While the mechanical strength against adhesive failure is maximum at the gel point, the mechanical strength against cohesive failure is still relatively low since the polymer at the gel point is only slightly crosslinked. As the crosslink density is increased beyond the gel point, the strength of the network increases (stronger cohesion) while the adhesive strength decreases. The distance of a polymer from the gel point therefore is expected to define the ratio of adhesive to cohesive strength. This general behavior was confirmed by Zosel [167] in his study of tack and peel behavior of radiation-crosslinked PDMS. He found the maximum tack (corresponding to adhesive strength) slightly after the gel point.

The knowledge of gelation leads to an unconventional but systematic approach to the development of pressure-sensitive adhesives. Based on the fact that gelation is a critical phenomenon, we hypothesize that there exists a universal 'law' which relates the adhesive to the cohesive properties at the gel point and in its vicinity. More research is needed for verifying this hypothesis and exploring its limits. For that purpose, polymer gels with well-defined chemical composition should be prepared at known distance from the gel point and their adhesive and rheological properties measured. The main parameters are: solid surface properties (chemical composition, homogeneity, regularity, roughness, curvature), chemical composition, wetting (phase diagram), and layer thickness.

The molecular weight is one of the most important parameters of polymer adhesion. Molecules of low molecular weight are able to wet a surface without adhering there for a longer period of time. There exists a chemistry-specific molecular weight beyond which molecules adhere to a surface. The transition from non-adhering to adhering may be described as a critical phenomenon. This same phenomenon has important implications for gelation near a surface: The molecular weight increases during gelation, and, at a certain extent of crosslinking, the largest cluster exceeds the critical molecular weight and adheres to the surface. This may have interesting consequences which should be explored further. The largest clusters may separate from the bulk, and the gel point will then be postponed in a very thin film while a solid layer is formed near the surface. Beyond the gel point, large molecules will not be able to move to the surface and the system will gradually feel 'dry', i.e. will not be able to stick to a surface.

Molecular orientation at the surface may also be important. A molecule orients planarly when deposited on a solid surface. Molecular strands prefer to be parallel to the surface; their probability of being oriented normal to the surface is very low. Several mechanisms can cause this orientation: (1) Surfaceactive sites may favor entire chain segments to interact with the surface. (2) The 
orientation is generated during the drying of the solution at the surface. A volume element of solution at the surface may initially contain isotropic polymer chains. Extraction of the solvent results in a shrinkage of the volume element into a thin film at the surface. Motion of the chain segments during this 'deformation' results in planar orientation.

\subsection{Processing Near the Physical Gel Point}

Many polymers solidify into a semi-crystalline morphology. Their crystallization process, driven by thermodynamic forces, is hindered due to entanglements of the macromolecules, and the crystallization kinetics is restricted by the polymer's molecular diffusion. Therefore, crystalline lamellae and amorphous regions coexist in semi-crystalline polymers. The formation of crystals during the crystallization process results in a decrease of molecular mobility, since the crystalline regions act as crosslinks which connect the molecules into a sample spanning network.

Injection molding of semi-crystalline polymers is an example of a process which is dominated by crystallization. Particularly the rate of crystallization is important. During injection of the molten polymer into the mold, a layer of polymer gets deposited at the cold walls of the mold where it starts solidifying while exposed to high shear stress due to ongoing injection (Fig. 27). This is an important part of the process, since the wall region later forms the surface of the manufactured product. A high shear stress during this crystallization might rupture the already solidified surface layers, resulting in surface defects (Y.G. Lin, personal communication). This could be avoided if the crystallization behavior were known together with its effect on the developing strength of the material. The polymer could to be modified to adjust its crystallization behavior

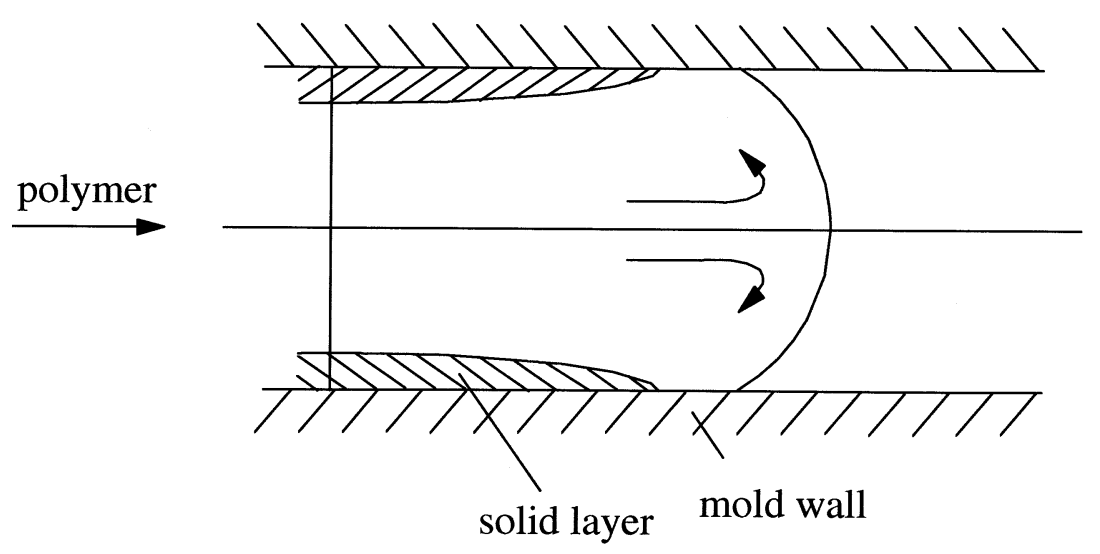

Fig. 27. Schematic of molten polymer flow during injection molding into a cold mold 
or a different polymer could be used or developed. A suitable tool to monitor the time dependence of crystallization processes and thus to select the best material and processing conditions is rheology. The characteristic crystallization time for each material can be tailored to the given molding circumstances.

\subsection{Processing Near the Chemical Gel Point}

Novel materials can be produced by crosslinking a polymer while shearing it at the same time. This process is known as dynamic vulcanization and is already used in the manufacture of certain rubbers and elastomers. At low degrees of crosslinking, the molecular structure is still very soft, so that the rate of molecular breaking can be of the same order as the rate of forming new chemical bonds. The result is a highly branched molecular structure with molecules of broadly distributed size. The materials have advantageous properties for applications as adhesives, damping materials with extremely broad damping maxima, toner for copy machines, superabsorbers, and sealants. The process gives access to materials with a wide range of properties through well-defined processing but to uniform chemical composition (mono-material, easy to recycle).

\section{Conclusions}

Polymers during their liquid-solid transitions develop a universal rheological behavior which is distinct from that of liquids or solids. It seems that this is a new, general phenomenon of nature. The phenomenological picture of this relaxation pattern is fairly complete. This allows the direct measurement of the liquid-solid transition during the manufacturing of gels and suggests a framework for presenting experimental data on gels. However, much too little is known yet about the molecular origin of the observed phenomena. Such molecular understanding would be desirable from a fundamental point of view, and it would be good to have when designing gels for specific applications.

We have further evidence that, in the absence of competing phase transitions, the material at the liquid-solid transition exhibits self-similar rheological behavior. The self-similarity expresses itself in power law relaxation and retardation spectra in the terminal zone. This very distinct relaxation pattern was first detected for chemically gelling systems. For these systems, a three-dimensional network structure is built by permanently connecting molecules through covalent bonds, and the longest relaxation time diverges at the gel point. However, the power law relaxation behavior seems to also govern physical gelation as long as the gel number is large, $N_{\mathrm{g}} \gg 1$, i.e. the lifetime of the junctions (which are reversible in contrast to chemical gelation) is long compared to the experimental observation time. This can be clearly seen at early 
stages of crystallization where a molecular network is formed with crystalline junctions. Furthermore, other physical mechanisms which lead to increasing correlation length seem to result in the same general relaxation patterns near the liquid/solid transition point.

The dynamic properties depend strongly on the material composition and structure. This is not included in current theories, which seem much too ideal in view of the complexity of the experimentally found relaxation patterns. Experimental studies involving concurrent determination of the static exponents, $d_{\mathrm{f}}$ and $\tau$, and the dynamic exponent, $n$, are required to find limiting situations to which one of the theories might apply.

The criticality of the liquid-solid transition does not necessarily imply universality for all investigated properties. Prefactors of different properties always depend on the details of the underlying structure, e.g. the critical gel stiffness is seen to vary by five orders of magnitude. Scaling of different static properties around a critical point is only universal if the structure growth process belongs to the same class (universality class). Dynamic scaling properties such as the power law relaxation exponent are much more complex and not expected to be universal. Values of $n$ as reported in this study, $0.19<n<0.92$, have been established over almost the entire possible range $(0<n<1)$. Stoichiometry, molecular weight, and concentration were all shown to have an impact on the critical gel properties. These critical gel properties are also coupled for the polymers studied here.

From a practical point of view, it is advantageous that critical gel properties depend on molecular parameters. It allows us to prepare materials near the gel point with a wide range of properties for applications such as adhesives, absorbents, vibration dampers, sealants, membranes, and others. By proper molecular design, it will be possible to tailor network structures, relaxation character, and the stiffness of gels to one's requirements.

Acknowledgements We gratefully acknowledge financial support from CUMIRP, MRSEC, and the National Science Foundation under grants NSF-MSM-86-01595 and NSF-DMR-88-06556. One of us, M.M., is indebted to the German Academic Exchange Service for financial support (DAADDoktorandenstipendium aus Mitteln des zweiten Hochschulsonderprogramms). We would also like to thank K. Dušek and K. te Nijenhuis for critically reading this text and for their helpful comments and suggestions.

\section{References}

1. Flory PJ (1941) J Am Chem Soc 63: 3083

2. Stockmayer WH (1943) J Chem Phys 11: 45

3. Stauffer D (1985) Introduction to percolation theory. Taylor and Francis, Philadelphia, USA

4. Vilgis TA, Winter HH (1988) Coll Polym Sci 266: 494

5. Chambon F, Winter HH (1985) Polym Bull 13: 499

6. Winter HH, Chambon F (1986) J Rheol 30: 367

7. Chambon F, Winter HH (1987) J Rheol 31: 683 
8. Baumgärtel M, Winter HH (1989) Rheol Acta 28: 511

9. Chasset R, Thirion P (1965) In: JA Prins (ed) Physics of non-crystalline solids. North-Holland, Amsterdam

10. Ferry JD (1980) Viscoelastic properties of polymers. Wiley, New York

11. Pipkin AC (1986) Lectures on viscoelasticity theory, 2nd edn. Springer, Berlin Heidelberg, New York

12. Orbey N, Dealy JM (1991) J Rheol 35: 1035

13. Martin JE, Adolf D, Wilcoxon JP (1989) Phys Rev A 39: 1325

14. Friedrich C, Heymann L (1988) J Rheol 32: 235; Friedrich C, Heymann L, Berger HR (1989) Rheol Acta 28: 535

15. Adolf D, Martin JE (1990) Macromolecules 23: 3700

16. Stauffer D, Coniglio A, Adam A (1982) Adv Polym Sci 44: 103

17. Winter HH, Izuka A, De Rosa ME (1994) Polym Gels Networks 2: 239

18. Winter HH (1987) Progr Colloid Polym Sci 75: 104

19. Winter HH (1991) MRS Bulletin 16(8): 44

20. Goldbart P, Goldenfeld N (1992) Phys Rev A 45: R5343

21. Vilgis TA (1992) Polymer Networks - Crosslinking, Rubbers, Microgels Ch. 33

22. Flory PJ (1953) Principles of polymer chemistry. Cornell University Press, Ithaca, New York

23. Miller DR, Macosko CW (1976) Macromolecules 9: 206

24. Miller DR, Vallés EM, Macosko CW (1979) Polym Eng Sci 19: 272

25. Dusek K, Ilavsky M (1974) J Polym Sci C 53: 57

26. Stanford JL, Stepto RFT, Waywell DR (1975) Faraday Disc 71: 1308

27. Gordon M, Scantlebury GR (1996) Proc Roy Soc London A292: 380

28. Gordon M, Templ WB (1972) Makromol Chem 160: 263

29. Vallés EM, Macosko CW (1979) Macromolecules 12: 521

30. Venkataraman SK, Coyne L, Chambon F, Gottlieb M, Winter HH (1989) Polymer 30: 2222

31. De Rosa ME (1994) Dissertation. University of Massachusetts at Amherst

32. Broadbent SR, Hammersley JM (1957) Proc Cambridge Phil Soc 53: 629

33. de Gennes PG (1979) Scaling concepts in polymer physics. Cornell, Ithaca, New York

34. Martin JE, Adolf D (1991) Annu Rev Phys Chem 42: 311

35. Colby RH, Rubinstein M, Gillmor JR, Mourey TH (1992) Macromolecules 25: 7180

36. Schosseler S, Leibler L (1984) Physique Lett 45: 501

37. Stauffer D (1981) Pure Appl Chem 53: 1479

38. Colby RH, Gillmor JR, Rubinstein M (1993) Phys Rev E 48: 3712

39. Martin JE, Adolf D, Wilcoxon JP (1988) Phys Rev Lett 61: 2620

40. Daoud M (1988) J Phys A 21: L237

41. de Gennes PG (1977) J Physique Lett 38: L-355

42. Muthukumar M, Winter HH (1986) Macromolecules 19: 1284

43. Hess W, Vilgis TA, Winter HH (1988) Macromolecules 21: 2536

44. Muthukumar M (1989) Macromolecules 22: 4656

45. Smoluchowski MV (1916) Phys Z 17: 585

46. Drake RL (1972) In: GM Hidy, JR Brock (ed) Topics in current aerosol research, vol 3. Pergamon, New York

47. Ernst MH (1984) In: EGD Cohen (ed) Fundamental problems in statistical mechanisms, vol. VI North-Holland, Amsterdam

48. van Dongen PGJ, Ernst MH (1985) Phys Rev Lett 54: 1396

49. Hendricks EM, Ernst MH, Ziff RM (1983) J Stat Phys 31: 519

50. Ziff RM, Ernst MH, Hendricks EM (1983) J Phys A 16: 2293

51. Leung YK, Eichinger BE (1984) J Chem Phys 80: 3877; (1984) J Chem Phys 80: 3885

52. Shy LY, Eichinger BE (1985) Brit Polym J 17: 200

53. Shy LY, Leung YK, Eichinger BE (1985) Macromolecules 18: 983

54. Bird RB, Armstrong RW, Hassager O (1987) Dynamics of Polymerics Liquids, vol 1. Wiley, New York

55. Lodge AS (1964) Elastic liquids: an introductory vector treatment of finite-strain polymer rheology. Academic, London

56. Chang H, Lodge AS (1972) Rheol Acta 11: 127

57. Larson R (1987) Constitutive equations for polymer melts and solutions. Butterworth, London

58. Scanlan JC, Winter HH (1991) Makrom Chem, Makrom Symp 45: 11

59. Izuka A, Winter HH, Hashimoto T (1992) Macromolecules 25: 2422 
60. De Rosa ME, Winter HH (1994) Rheol Acta 33: 220

61. Baumgärtel M, Schausberger A, Winter HH (1990) Rheol Acta 29: 400

62. Baumgärtel M, De Rosa ME, Machado J, Masse M, Winter HH (1992) Rheol Acta 31: 75

63. Hogdson DF, Amis EJ (1990) Macromolecules 23: 2512

64. Lairez D, Adam M, Emery JR, Durand D (1992) Macromolecules 25: 286

65. Muller R, Gérard E, Dugand P, Rempp P, Gnanou Y (1991) Macromolecules 24: 1321

66. Durand D, Delsanti M, Adam M, Luck JM (1987) Europhys Lett 3: 297

67. te Nijenhuis K, Winter HH (1989) Macromolecules 22: 411

68. Lin YG, Mallin DT, Chien JCW, Winter HH (1991) Macromolecules 24: 850

69. Richtering HW, Gagnon KD, Lenz RW, Fuller RC, Winter HH (1992) Macromolecules 25: 2429

70. Mours M, Winter HH (1996) Macromolecules 29: 7221

71. Venkataraman SK, Winter HH (1990) Rheol Acta 29: 423

72. Cole KS, Cole RH (1941) J Chem Phys 9: 341

73. Gross B (1953) Mathematical structures of the theory of viscoelasticity. Hermann, Paris

74. Cates ME (1987) Macromolecules 20: 2289

75. Cates ME, Candau SJ (1990) J Phys: Condens Matter 2: 6869

76. te Nijenhuis K (1995) Viscoelastic properties of thermoreversible gels, submitted to Adv Polym Sci

77. Keller A (1995) Faraday Disc 101: 1

78. Lehsaini N, Muller R, Weill G, François J (1994) Polymer 35: 2180

79. Yu Q, Amis EJ (1993) Makromol Chem, Makromol Symp 76: 193

80. Schwittay C, Mours M, Winter HH (1995) Faraday Disc 101: 93

81. Guenet JM (1992) Thermoreversible gelation of polymers and biopolymers. Academic, London

82. Reid DS, Bryce TA, Clark AH, Rees DA (1974) Faraday Disc 57: 230

83. Ross-Murphy SB (1991) Rheol Acta 30: 401

84. Michon C, Cuvelier G, Launay B (1993) Rheol Acta 32: 94

85. Wedler W, Tang W, Winter HH, MacKnight WJ, Farris RJ (1995) Macromolecules 28: 512

86. Winter HH, Scott DB, Gronski W, Okamoto S, Hashimoto T (1993) Macromolecules 26: 7236

87. Larson RG, Winey KI, Patel SS, Watanabe H, Bruinsma R (1993) Rheol Acta 32: 245

88. Vasilev VG, Rogovina LZ, Slonimskii GL, Papkov VS, Shchegolikhina OI, Zhdanov AA (1995) Polym Sci, Ser A 37: 174

89. Rogovina LZ, Vasilev VG, Papkov VS, Shchegolikhina OI, Slonimskii GL, Zhdanov AA (1995) Macromol Symp 93: 135

90. Tobolsky AV, McLouhglin JR (1952) J Polym Sci 8: 543

91. Cheng YT, Johnson WL (1987) Science 235: 997

92. Zorn R, McKenna GB, Willner L, Richter D (1995) Macromolecules 28: 8552

93. Osaki K, Inoue T, Hwang EJ, Okamoto H, Takiguchi O (1994) J Non-cryst Solids 172-174: 838

94. Colby RH, Gillmor JR, Galli G, Laus M, Ober CK, Hall E (1993) Liquid Cryst 13: 233

95. Rubin SF, Kannan RM, Kornfield JA, Boeffel C (1994) Proc ACS Polym Mater Sci Eng 71: 330,486

96. Münstedt H (1975) Angew Makrom Chem 47: 229

97. Zosel A (1972) Rheol Acta 11: 229

98. Laun HM (1984a) Angew Makromol Chem 123/124: 335

99. White JL (1979) J Non-Newt Fluid Mech 5: 177

100. Buscall R, Goodwin JW, Hawkins MW, Ottewill RH (1982) J Chem Soc Faraday Trans I 78: 2873, 2889

101. Münstedt H (1981) Polym Eng Sci 21: 259

102. Castellani L, Lomellini P (1991) Plastics, Rubber and Composites Processing and Applications 16: 25

103. Minagawa N, White JL (1975) Polym Eng Sci 15: 825

104. Krieger IM (1972) Adv Colloid Interface Sci 3: 111

105. Krieger IM, Eguiluz M (1976) Trans Soc Rheol 20: 29

106. Hoffmann RL (1972) Trans Soc Rheol 16: 155

107. Hoffmann RL (1974) J Colloid Interface Sci 46: 491

108. Jeffrey DJ, Acrivos A (1976) AIChE J 22: 417

109. Russel WB, Saville DA, Schowalter WR (1989) Colloidal Dispersions. Cambridge University Press, Cambridge 
110. Laun HM (1984) Colloid Polym Sci 262: 257

111. Metzner AB (1985) J Rheol 29: 739

112. Masuda T, Nakajima A, Kitamura M, Aoki Y, Yamauchi N, Yoshioka A (1984) Pure Appl Chem 56: 1457

113. Weinberger CB, Goddard JD (1974) Intern J Multiphase Flow 1: 465

114. Castellani L, Lomellini P (1994) Rheol Acta 33: 446

116. Baumgärtel M, Winter HH (1992) J Non-Newt Fluid Mech 44: 15

115. Venkataraman SK (unpublished results)

117. Mours M, Winter HH (1994) Rheol Acta 33: 385

118. te Nijenhuis K, Dijkstra D (1975) Rheol Acta 14: 71

119. Winter HH, Morganelli P, Chambon F (1988) Macromolecules 21: 532

120. Holly EE, Venkataraman SK, Chambon F, Winter HH (1988) J Non-Newt Fluid Mech 27: 17

121. Izuka A, Winter HH, Hashimoto T (1994) Macromolecules 27: 6883

122. Scanlan JC, Winter HH (1991) Macromolecules 24: 47

123. Flory PJ (1974) Faraday Disc 57: 7

124. Schmidt M, Burchard W (1981) Macromolecules 14: 370

125. Martin JE, Wilcoxon JP, Adolf D (1987) Phys Rev A 36: 1803

126. Martin JE, Wilcoxon JP (1988) Phys Rev Lett 61: 373

127. Martin JE, Keefer KD (1986) Phys Rev A 34: 4988

128. Kajiwara K, Burchard W, Kowalski M, Nerger D, Dusek K, Mateijka L, Tuzar Z (1984) Makromol Chem 185: 2543

129. Vallés EM, Macosko CW (1979) Macromolecules 12: 673

130. Allain C, Amiel C (1986) Phys Rev Lett 56: 1501

131. Barton JM, Buist GJ, Hamerton I, Howlin BJ, Jones RJ, Lin S (1994) Polym Bull 33: 215

132. Mijovic J, Kim J, Slaby J (1984) J Appl Polym Sci 29: 1449

133. Muzumdar SV, Lee LJ (1991) Polym Eng Sci 31: 1647

134. Lee JH, Lee JW (1994) Polym Eng Sci 34: 742

135. Soltero J, González-Romero V (1988) Proc Ann Techn Meet Soc Plast Eng, ANTEC, pp 1057-1061

136. Walton JR, Williams KPJ (1991) Vibr Spectr 1: 339

137. Lyon RE, Chike KE, Angel SM (1994) J Appl Polym Sci 53: 1805

138. Macosko CW (1985) Brit Polym J 17: 239

139. Lipshitz S, Macosko CW (1976) Polym Eng Sci 16: 803

140. Vallés EM, Macosko CW (1976) Rubber Chem Tech 49: 1232

141. Castro JM, Macosko CW, Perry SJ (1984) Polym Commun 25: 82

142. Apicella A, Masi P, Nicolais L (1984) Rheol Acta 23: 291

143. Adam M, Delsanti M, Durand D (1985) Macromolecules 18: 2285

144. Malkin AY (1985) Plaste Kautschuk 32: 281

145. Bidstrup SA (1986) Dissertation University of Minnesota

146. Allain C, Salomé L (1987) Polym Commun 28: 109

147. Axelos MAV, Kolb M (1990) Phys Rev Lett 64: 1457

148. Farris RJ, Lee C (1983) Polym Eng Sci 23: 586

149. Winter HH (1987) Polym Eng Sci 27: 1698

150. Cuvellier G, Peighy-Nourry C, Launay B (1990) In: S Phillips (ed) Gums and stabilizers for the food industry. IRL, Oxford, UK, pp 549-552

151. Malkin AY, Kulichikhin SG (1991) Adv Polym Sci 101: 217

152. Gillham JK (1976) Adv Polym Sci 19: 319; (1976) Adv Polym Sci 19: 676

153. ASTM Standard (1985) D4473-85

154. Tung CYM, Dynes PJ (1982) J Appl Polym Sci 27: 569

155. Antonietti M, Fölsch KF, Sillescu H, Pakula T (1989) Macromolecules 22: 2812

156. Bates FS (1984) Macromolecules 17: 2607

157. Larson R (1985) Rheol Acta 24: 327

158. Roovers J, Graessley WW (1981) Macromolecules 14: 766

159. Cates ME (1985) J Physique 46: 1059

160. Muthukumar M (1985) J Chem Phys 83: 3161

161. Andrews RD, Tobolsky AV (1951) J Polym Sci 7: 221

162. Gouinlock EV, Porter RS (1977) Polym Eng Sci 17: 535

163. Chung CI, Lin MI (1978) J Polym Sci, Polym Phys Ed 16: 545 
164. Winter HH (1994) J Non-cryst solids 172-174: 1158

165. Vallés EM, Carella JM, Winter HH, Baumgärtel M (1990) Rheol Acta 29: 535

166. Khan SA, Plitz IM, Frank RA (1992) Rheol Acta 31: 151

167. Zosel A (1991) J Adhesion 34: 201

Editor: Prof. K. Dusek

Received: October 1996 